\title{
The $C^{1}$ generic diffeomorphism has trivial centralizer
}

\author{
C. Bonatti, S. Crovisier and A. Wilkinson
}

October 23, 2018

\begin{abstract}
Answering a question of Smale, we prove that the space of $C^{1}$ diffeomorphisms of a compact manifold contains a residual subset of diffeomorphisms whose centralizers are trivial.
\end{abstract}

\section{Contents}

1 Introduction 3

1.1 The centralizer problem, and its solution in $\operatorname{Diff}^{1}(M) \ldots \ldots \ldots$

1.2 The algebraic structure and the topology of $\operatorname{Diff}^{1}(M) \ldots \ldots$. . . 4

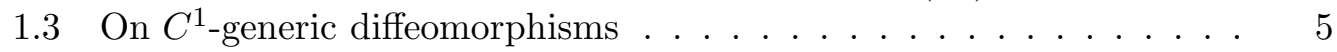

1.3.1 A special property of the $C^{1}$-topology . . . . . . . . 5

1.3.2 Perturbing the derivative without changing the topological dynamics . . . . . . . . . . . . . . . . 6 6

1.3.3 Tidy perturbations . . . . . . . . . . . . 7

1.4 Local and global: the structure of the proof of the Main Theorem . . 8

1.5 Perturbations for obtaining the (LD) and (UD) properties . . . . . 9

2 The local and global strategies 10

2.1 Background on $C^{1}$-generic dynamics . . . . . . . . . . . . . 10

2.2 Conditions for the local strategy: the unbounded distortion (UD) properties ............................ 11

2.3 Condition for the global strategy: the large derivative (LD) property 12

2.4 Checking that the centralizer is trivial . . . . . . . . . 13

2.5 From dense to residual: compactness and semicontinuity . . . . . 15

3 Perturbing the derivative without changing the dynamics 17

3.1 Tidy perturbations . . . . . . . . . . . . . . . . . 17

3.2 Sequences of tidy perturbations . . . . . . . . . . . 18

3.3 Topological towers . . . . . . . . . . . . . . . . 20

3.4 Towers avoiding certain sets . . . . . . . . . . . . . 20

3.5 Linearizing the germ of dynamics along a nonperiodic orbit . . . . 23 
4 (LD) property: reduction to a perturbation result in towers 25

4.1 Our shopping list for the proof of Theorem B . . . . . . . . . 25

4.2 A priori choices . . . . . . . . . . . . . 26

4.2.1 Choice of $\varepsilon_{i}, n_{i}, m_{i}, M_{i} \ldots \ldots \ldots \ldots$

4.2.2 Choice of $x_{i}, Z_{i}$ and $N_{i} \ldots \ldots \ldots \ldots \ldots$

4.3 Inductive hypotheses implying Theorem B . . . . . . . . . . . . 28

4.3.1 Conditions on $\rho_{i}$ so that $\left(\Phi_{i}\right)$ converges $\ldots \ldots \ldots 28$

4.3.2 Conditions on $\rho_{i}$ preserving prolonged visits to towers . . . 28

4.3.3 Conditions on $\rho_{i}, U_{i}$ and $g_{i}$ for the (LD) property . . . . . 29

4.3.4 The derivative at the periodic orbits is preserved . . . . . . 30

4.3.5 Conditions on $U_{i}$ for preserving the (UD) property . . . . . . 30

4.4 Satisfying the inductive hypotheses . . . . . . . . . . . 32

5 Large derivative by perturbation in towers 33

5.1 Reduction to cocycles . . . . . . . . . . . . . 33

5.2 Reduction to a perturbation result in a cube . . . . . . . . 35

5.3 Reduction to linear perturbations . . . . . . . . . . . . . 37

5.4 Huge versus bounded intermediary products . . . . . . . . . . . . 39

6 (UD) property: reduction to a perturbation result in a cube 40

6.1 Reduction to a perturbation result in towers . . . . . . . . . . . . . 40

6.2 Localization of the perturbation . . . . . . . . . . . . 43

6.3 Reduction to cocycles . . . . . . . . . . . . . . 45

6.4 Reduction to a perturbation result in a cube . . . . . . . 47

7 Almost tidy perturbation in a cube $\quad 50$

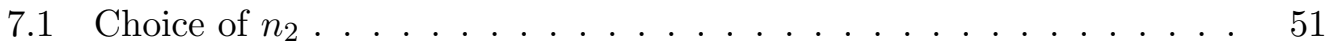

7.2 Construction of a perturbation ................ 51

7.3 Support of the perturbation: first choice of $\mu \ldots \ldots \ldots 2$

7.4 Size of the perturbation: second choice of $\mu \ldots \ldots . \ldots 53$

7.5 Perturbation of the jacobian: third choice of $\mu \ldots \ldots \ldots$

7.6 Almost tidy perturbation: fourth choice of $\mu \ldots \ldots . \ldots 5$

Appendix: The (LD) property is not generic 56 


\section{Introduction}

\subsection{The centralizer problem, and its solution in $\operatorname{Diff}^{1}(M)$}

It is a basic fact from linear algebra that any two commuting matrices in $G L(n, \mathbb{C})$ can be simultaneously triangularized. The reason why is that if $A$ commutes with $B$, then $A$ preserves the generalized eigenspaces of $B$, and vice versa. Hence the relation $A B=B A$ is quite special among matrices, and one might not expect it to be satisfied very often. In fact, it's not hard to see that the generic pair of matrices $A$ and $B$ generate a free group; any relation in $A$ and $B$ defines a nontrivial subvariety in the space of pairs of matrices, so the set of pairs of matrices that satisfy no relation is residual: it contains a countable intersection of open-dense subsets. On the other hand, the generic $n \times n$ matrix is diagonalizable and commutes with an $n$-dimensional space of matrices. That is, a residual set of matrices have large centralizer.

Consider the same sort of questions, this time for the group of $C^{1}$ diffeomorphisms $\operatorname{Diff}^{1}(M)$ of a compact manifold $M$. If $f$ and $g$ are diffeomorphisms satisfying $f g=g f$, then $g$ preserves the set of orbits of $f$, as well as all of the smooth and topological dynamical invariants of $f$, and vice versa. Also in analogy to the matrix case, an easy transversality argument (written in [G, Proposition 4.5] for circle homeomorphisms), shows that for a generic $\left(f_{1}, \ldots, f_{p}\right) \in\left(\operatorname{Diff}^{r}(M)\right)^{p}$ with $p \geq 2$ and $r \geq 0$, the group $\left\langle f_{1}, \ldots, f_{p}\right\rangle$ is free. In contrast with the matrix case, however, the generic $C^{1}$ diffeomorphism cannot have a large centralizer. This is the content of this paper. Our main result is:

Main Theorem. Let $M$ be any closed, connected smooth manifold. There is a residual subset $\mathcal{R} \subset \operatorname{Diff}^{1}(M)$ such that for any $f \in \mathcal{R}$, and any $g \in \operatorname{Diff}^{1}(M)$, if $f g=g f$, then $g=f^{n}$, for some $n \in \mathbb{Z}$.

This theorem 11 gives an affirmative answer in the $C^{1}$ topology to the following question, posed by S. Smale. We fix a closed manifold $M$ and consider the space $\operatorname{Diff}^{r}(M)$ of $C^{r}$ diffeomorphisms of $M$, endowed with the $C^{r}$ topology. The centralizer of $f \in \operatorname{Diff}^{r}(M)$ is defined as

$$
Z^{r}(f):=\left\{g \in \operatorname{Diff}^{r}(M): f g=g f\right\} .
$$

Clearly $Z^{r}(f)$ always contains the cyclic group $\langle f\rangle$ of all the powers of $f$. We say that $f$ has trivial centralizer if $Z^{r}(f)=\langle f\rangle$. Smale asked the following:

Question 1 ([Sm1, Sm2]). Consider the set of $C^{r}$ diffeomorphisms of a compact manifold $M$ with trivial centralizer.

1. Is this set dense in $\operatorname{Diff}^{r}(M)$ ?

2. Is it residual in $\operatorname{Diff}^{r}(M)$ ? That is, does it contain a dense $G_{\delta}$ ?

3. Does it contain an open and dense subset of $\operatorname{Diff}^{r}(M)$ ?

\footnotetext{
${ }^{1}$ This result has been announced in BCW2.
} 
For the case $r=1$ we now have a complete answer to this question. The theorem above shows that for any compact manifold $M$, there is a residual subset of $\operatorname{Diff}^{1}(M)$ consisting of diffeomorphisms with trivial centralizer, giving an affirmative answer to the second (and hence the first) part of Question 11. Recently, with G. Vago, we have also shown:

Theorem. [BCVW] For any compact manifold $M$, the set of $C^{1}$ diffeomorphisms with trivial centralizer does not contain any open and dense subset.

This result gives a negative answer to the third part of Question 1; on any compact manifold, [BCVW] exhibits a family of $C^{\infty}$ diffeomorphisms with large centralizer that is $C^{1}$ dense in a nonempty open subset of $\operatorname{Diff}^{1}(M)$.

The history of Question 1 goes back to the work of N. Kopell [Ko], who gave a complete answer for $r \geq 2$ and the circle $M=S^{1}$ : the set of diffeomorphisms with trivial centralizer contains an open and dense subset of $\operatorname{Diff}^{r}\left(S^{1}\right)$. For $r \geq 2$ on higher dimensional manifolds, there are partial results with additional dynamical assumptions, such as hyperbolicity [PY1, PY2, Fi] and partial hyperbolicity Bu1]. In the $C^{1}$ setting, Togawa proved that generic Axiom A diffeomorphisms have trivial centralizer. In an earlier work [BCW1, we showed that for $\operatorname{dim}(M) \geq 2$, the $C^{1}$ generic conservative (volume-preserving or symplectic) diffeomorphism has trivial centralizer in $\operatorname{Diff}^{1}(M)$. A more complete list of previous results can be found in BCW1].

\subsection{The algebraic structure and the topology of $\operatorname{Diff}^{1}(M)$}

These results suggest that the topology of the set of diffeomorphisms with trivial centralizer is complicated and motivate the following questions.

Question 2. 1. Consider the set of diffeomorphisms whose centralizer is trivial. What is its interior?

2. Is it a Borel set? (See [FRW] for a negative answer to this question in the measurable context.)

3. The set $\left\{(f, g) \in \operatorname{Diff}^{1}(M) \times \operatorname{Diff}^{1}(M): f g=g f\right\}$ is closed. What is its local topology? For example, is it locally connected?

Beyond just proving the genericity of diffeomorphisms with trivial centralizer, we find a precise collection of dynamical properties of $f$ that imply that $\mathcal{Z}^{1}(f)=\langle f\rangle$. As an illustration of what we mean by this, consider the Baumslag-Solitar relation $g f g^{-1}=f^{n}$, where $n>1$ is a fixed integer. Notice that if this relation holds for some $g$, then the periodic points of $f$ cannot be hyperbolic. This implies that for the $C^{1}$ generic $f$, there is no diffeomorphism $g$ satisfying $g f g^{-1}=f^{n}$. In this work, we consider the relation $g f g^{-1}=f$. The dynamical properties of $f$ that forbid this relation for $g \notin\langle f\rangle$ are the Large Derivative (LD) and Unbounded Distortion (UD) properties (described in greater detail in the subsequent sections).

Hence the Main Theorem illustrates the existence of links between the dynamics of a diffeomorphism and its algebraic properties as a element of the group $\operatorname{Diff}^{1}(M)$. In that direction, it seems natural to propose the following (perhaps naive) generalizations of Question 1 . 
Question 3. 1. Consider $G=\left\langle a_{1}, \ldots, a_{k} \mid r_{1}, \ldots, r_{m}\right\rangle$ a finitely presented group where $\left\{a_{i}\right\}$ is a generating set and $r_{i}$ are relations. How large is the set of diffeomorphisms $f \in \operatorname{Diff}^{r}(M)$ such that there is an injective morphism $\rho: G \rightarrow \operatorname{Diff}^{r}(M)$ with $\rho\left(a_{1}\right)=f$ ? The Main Theorem implies that this set is meagre if $G$ is abelian (or even nilpotent).

2. Does there exist a diffeomorphism $f \in \operatorname{Diff}^{r}(M)$ such that, for every $g \in$ $\operatorname{Diff}^{r}(M)$, the group generated by $f$ and $g$ is either $\langle f\rangle$ if $g \in\langle f\rangle$ or the free product $\langle f\rangle *\langle g\rangle$ ?

3. Even more, does there exist a diffeomorphism $f \in \operatorname{Diff}^{r}(M)$ such that, for every finitely generated group $G \in \operatorname{Diff}^{r}(M)$ with $f \in G$, there is a subgroup $H \subset G$ such that $G=\langle f\rangle * H$ ?

4. If the answer to either of the two previous items is "yes", then how large are the corresponding sets of diffeomorphisms with these properties?

\subsection{On $C^{1}$-generic diffeomorphisms}

Our interest in Question 1 comes also from another motivation. The study of the dynamics of diffeomorphisms from the perspective of the $C^{1}$-topology has made substantial progress in the last decade, renewing hope for a reasonable description of $C^{1}$-generic dynamical systems. The elementary question on centralizers considered here presents a natural challenge for testing the strength of the new tools.

Our result certainly uses these newer results on $C^{1}$-generic dynamics, in particular those related to Pugh's closing lemma, Hayashi's connecting lemma, the extensions of these techniques incorporating Conley theory, and the concept of topological tower introduced in $[\mathrm{BC}$. Even so, the Main Theorem is far from a direct consequence of these results; in this subsection, we focus on the new techniques and tools we have developed in the process of answering Question 1 .

Our proof of the Main Theorem goes back to the property which is at the heart of most perturbation results specific to the $C^{1}$-topology.

\subsubsection{A special property of the $C^{1}$-topology}

If we focus on a very small neighborhood of an orbit segment under a differentiable map, the dynamics will appear to be linear around each point; thus locally, iterating a diffeomorphism consists in multiplying invertible linear maps.

On the other hand, the $C^{1}$-topology is the unique smooth topology that is invariant under (homothetical) rescaling. More precisely, consider a perturbation $f \circ g$ of a diffeomorphism $f$, where $g$ is a diffeomorphism supported in a ball $B(x, r)$ and $C^{1}$-close to the identity. The $C^{1}$-size of the perturbation does not increase if we replace $g$ by its conjugate $h_{\lambda} g h_{\lambda}^{-1}$ where $h_{\lambda}$ is the homothety of ratio $\lambda<1$. The new perturbation is now supported on the ball $B(x, r \lambda)$. When $\lambda$ goes to 0 , we just see a perturbation of the linear map $D_{x} f$.

This shows that local $C^{1}$-perturbation results are closely related to perturbations of linear cocycles. This connection is quite specific to the $C^{1}$-topology: this type of renormalization of a perturbation causes its $C^{2}$-size to increase proportionally to 
the inverse of the radius of its support. The rescaling invariance property of the $C^{1}$ topology is shared with the Lipschitz topology; however, bi-Lipschitz homeomorphisms do not look like linear maps at small scales.

This special property of the $C^{1}$-topology was first used in the proof of Pugh's closing lemma, and perhaps the deepest part of the proof consists in understanding perturbations of a linear cocycle. Pugh introduced the fundamental, simple idea that, if we would like to perform a large perturbation in a neighborhood of a point $x$, we can spread this perturbation along the orbit of $x$ and obtain the same result, but by $C^{1}$-small perturbations supported in the neighborhood of an orbit segment. The difficulty in this idea is that, if one performs a small perturbation of $f$ in a very small neighborhood of the point $f^{i}(x)$, the effect of this perturbation when observed from a small neighborhood of $x$ is deformed by a conjugacy under the linear map $D f^{i}$; this deformation is easy to understand if $D f^{i}$ is an isometry or a conformal map, but it has no reason in general to be so.

Our result is based on Propositions 5.2 and 6.6, which both produce a perturbation of a linear cocycle supported in the iterates of a cube $Q$. The aim of Proposition 5.2 is to perturb the derivative $D f$ in order to obtain a large norm $\left\|D f^{n}\right\|$ or $\left\|D f^{-n}\right\|$, for a given $n$ and for every orbit that meets a given subcube $\delta Q \subset Q$; the aim of Proposition 6.6 is to obtain a large variation of the jacobian $\operatorname{det} D f^{n}$, for some integer $n$ and for all the points of a given subcube $\theta Q \subset Q$. The first type of perturbation is connected to the Large Derivative (LD) property, and the second, to the Unbounded Distortion (UD) property.

The main novelty of these two elementary perturbation results is that, in contrast to the case of the closing lemma, whose aim is to perturb a single orbit, our perturbation lemmas will be used to perturb all the orbits of a given compact set. To perturb all orbits in a compact set, we first cover the set with open cubes, and then carry out the perturbation cube-by-cube, using different orbit segments for adjacent cubes. For this reason, we need to control the effect of the perturbation associated to a given cube on the orbits through all of its neighboring cubes.

To obtain this control, for each cube in the cover, we perform a perturbation along the iterates of the cubes until we obtain the desired effect on the derivative, and then we use more iterates of the cube to "remove the perturbation." By this method, we ensure that the long-term effect of the perturbation on the orbit will be as small as possible in the case of Proposition 6.6, and indeed completely removed in the case of Proposition 5.2. In the latter case, we speak of tidy perturbations. These perturbations, which are doing "nothing or almost nothing," are our main tools.

\subsubsection{Perturbing the derivative without changing the topological dy- namics}

In the proof of the Main Theorem, we show that every diffeomorphism $f$ can be $C^{1}$-perturbed in order to obtain simultaneously the (UD)- and (LD)-properties, which together imply the triviality of the centralizer. However, unlike the (UD)property, the (LD)-property is not a generic property: to get both properties to hold simultaneously, we have to perform a perturbation that produces the (LD)property while preserving the (UD)-property. 
Our solution consists in changing the derivative of $f$ without changing its topological dynamics. The only context in which an arbitrary perturbation of the derivative of $f$ will not alter its dynamics is one where $f$ is structurally stable. Here $f$ is not assumed to have any kind of stability property, and yet we can realize a substantial effect on the derivative by a perturbation preserving the topological dynamics. For example, starting with an irrational rotation of the torus $\mathbb{T}^{d}$, we can obtain, via an arbitrarily $C^{1}$-small perturbation, a diffeomorphism $g$, conjugate to the original rotation, with the property:

$$
\lim _{n \rightarrow \infty} \inf _{x \in \mathbb{T}^{d}} \sup _{y \in \operatorname{orb}_{g}(x)}\left\|D g^{n}(y)\right\|+\left\|D g^{-n}(y)\right\|=\infty .
$$

Let us state our result (this is a weak version of Theorem B below):

Theorem. Let $f$ be a diffeomorphism whose periodic orbits are all hyperbolic. Then any $C^{1}$-neighborhood of $f$ contains a diffeomorphism $g$ such that

- $g$ is conjugate to $f$ via a homeomorphism.

- $g$ has the large derivative property: for every $K>0$ there exists $n_{K}$ such that, for every $n \geq n_{K}$ and any non-periodic point $x$ :

$$
\sup _{y \in \operatorname{orb}_{g}(x)}\left\{\left\|D g^{n}(y)\right\|,\left\|D g^{-n}(y)\right\|\right\}>K \text {. }
$$

As far as we know, this result is the first perturbation lemma which produces a perturbation of the derivative inside the topological conjugacy class of a given diffeomorphism (with the unique hypothesis that all periodic orbits are hyperbolic, which is generic in any topology). In the proof, we construct the perturbation $g$ of $f$ as a limit of tidy perturbations $g_{n}$ of $f$ which are smoothly conjugate to $f$. As we think that tidy perturbations will used in further works, we present them in some detail.

\subsubsection{Tidy perturbations}

Let $f$ be a diffeomorphism and let $U$ be an open set such that the first iterates $\bar{U}$, $f(\bar{U}), \ldots, f^{n}(\bar{U})$ are pairwise disjoint, for some $n>0$. Consider a perturbation $g$ of $f$ with support in $V=\bigcup_{0}^{n-1} f^{i}(U)$ that has no effect at all on the orbits crossing $V$ : that is, for every $x \in U, f^{n}(x)=g^{n}(x)$. In our proof of Theorem B, we construct such perturbations, using the first iterates $\bar{U}, f(\bar{U}), \ldots, f^{i}(\bar{U})$, for some $i \in\{0, \ldots, n-2\}$, for perturbing $f$ and getting the desired effect on the derivative, and using the remaining iterates $f^{i+1}(\bar{U}), \ldots, f^{n-1}(\bar{U})$ for removing the perturbation, bringing the $g$-orbits of the points $x \in \bar{U}$ back onto their $f$-orbits. The diffeomorphism $g$ is smoothly conjugate to $f$ via some diffeomorphism that is the identity map outside $\bigcup_{0}^{n-1} f^{i}(U)$. Such a perturbation is called a tidy perturbation.

Tidy perturbations require open sets that are disjoint from many of their iterates. To get properties to hold on all non-periodic points, we use such open sets that also cover all the orbits of $f$, up to finitely many periodic points of low period. Such 
open sets, called topological towers, were constructed in [BC] for the proof of a connecting lemma for pseudo-orbits. In Section 3.4, we further refine the construction of topological towers; in particular, we prove that it is always possible to construct topological towers disjoint from any compact set with the wandering orbit property. The wandering orbit property is a weak form of wandering behavior, satisfied, for example, by nonperiodic points and compact subsets of the wandering set.

In the proof of Theorem B, we construct an infinite sequence $\left(g_{i}\right)$, where $g_{i}$ is a tidy perturbation of $g_{i-1}$, with support in the disjoint $n_{i}$ first iterates of some open set $U_{i}$. We show that, if the diameters of the $U_{i}$ decrease quickly enough, then the conjugating diffeomorphisms converge in the $C^{0}$-topology to a homeomorphism conjugating the limit diffeomorphism $g$ to $f$. With a weaker decay to 0 of the diameters of the $U_{i}$, it may happen that the conjugating diffeomorphisms converge uniformly to some continuous noninvertible map. In that case, the limit diffeomorphism $g$ is merely semiconjugate to $f$. This kind of technique has already been used, for instance by M. Rees [R], who constructed homeomorphisms of the torus with positive entropy and semiconjugate to an irrational rotation.

Controlling the effect of successive general perturbations is very hard. For tidy perturbations it is easier to manage the effect of a successive sequence of them, since each of them has no effect on the dynamics. However this advantage leads to some limitations on the effect we can hope for, in particular on the derivative. We conjecture for instance that it is not possible to change the Lyapunov exponents:

Conjecture 1.1. Let $g=h f h^{-1}$ be a limit of tidy perturbations $g_{i}=h_{i} f h_{i}^{-1}$ of $f$, where the $h_{i}$ converge to $h$. Then given any ergodic invariant measure $\mu$ of $f$, the Lyapunov exponents of the measure $h_{*}(\mu)$ for $g$ are the same as those of $\mu$ for $f$.

To obtain the (LD)-property, we create some oscillations in the size of the derivative along orbits. It seems natural to ask if, on the other hand, one could erase oscillations of the derivative. Let us formalize a question:

Question 4. Let $f$ be a diffeomorphism and assume that $\Lambda$ is a minimal invariant compact set of $f$ that is uniquely ergodic with invariant measure $\mu$. Assume that all the Lyapunov exponents of $\mu$ vanish. Does there exist $g=h f h^{-1}$, a limit of tidy perturbations of $f$, such that the norm of $D g^{n}$ and of $D g^{-n}$ remain bounded on $h(\Lambda)$ ?

Such minimal uniquely ergodic sets appear in the $C^{1}$ generic setting (among the dynamics exhibited by $[\mathrm{BD}]$ ).

In this paper, starting with a diffeomorphism $f$ without the (LD)-property, we build a perturbation $g=h f h^{-1}$ as a limit of tidy perturbations and such that $g$ satisfies the (LD)-property. Then the linear cocycles $D f$ over $f$ and $D g$ over $g$ are not conjugate by a continuous linear cocycle over $h$.

Question 5. Is there a measurable linear cocycle over $h$ conjugating $D g$ to $D f$ ?

\subsection{Local and global: the structure of the proof of the Main The- orem}

The proof of the Main Theorem breaks into two parts, a "local" one and a "global" one. This is also the general structure of the proofs of the main results in [Ko, PY1, 
PY2, To1, To2, Bu2]:

- The local part proves that for the generic $f$, if $g$ commutes with $f$, then $g=f^{\alpha}$ on an open and dense subset $W \subset M$, where $\alpha: W \rightarrow \mathbb{Z}$ is a locally constant function.

This step consists in "individualizing" a dense collection of orbits of $f$, arranging that the behavior of the diffeomorphism in a neighborhood of one orbit is different from the behavior in a neighborhood of any other. Hence $g$ must preserve each of these orbits, which allows us to obtain the function $\alpha$ on these orbits.

This individualization of orbits happens whenever a property of unbounded distortion (UD) holds between certain orbits of $f$, a property which we describe precisely in the next section. Theorem A shows that the (UD) property holds for a residual set of $f$.

- The global part consists in proving that for generic $f, \alpha$ is constant. We show that it is enough to verify that the function $\alpha$ is bounded. This would be the case if the derivative $D f^{n}$ were to take large values on each orbit of $f$, for each large $n$ : the bound on $D g$ would then forbid $\alpha$ from taking arbitrarily large values. Notice that this property is global in nature: we require large derivative of $f^{n}$ on each orbit, for each large $n$.

Because it holds for every large $n$, this large derivative (LD) property is not generic, although we prove that it is dense. This lack of genericity affects the structure of our proof: it is not possible to obtain both (UD) and (LD) properties just by intersecting two residual sets. Theorem B shows that among the diffeomorphisms satisfying (UD), the property (LD) is dense. This allows us to conclude that the set of diffeomorphisms with trivial centralizer is $C^{1}$ dense.

There is some subtlety in how we obtain a residual subset from a dense subset. Unfortunately we don't know if the set of diffeomorphisms with trivial centralizer form a $G_{\delta}$, i.e., a countable intersection of open sets. For this reason, we consider centralizers defined inside of the larger space of bi-Lipschitz homeomorphisms, and we use the compactness properties of this space. The conclusion is that if a $C^{1}$ dense set of diffeomorphisms has trivial centralizer inside of the space of bi-Lipschitz homeomorphisms, then this property holds on a $C^{1}$ residual set.

\subsection{Perturbations for obtaining the (LD) and (UD) properties}

To complete the proof of the Main Theorem, it remains to prove Theorems A and B. Both of these results split in two parts.

- The first part is a local perturbation tool, which changes the derivative of $f$ in a very small neighborhood of a point, the neighborhood being chosen so small that $f$ resembles a linear map on many iterates of this neighborhood. 
- In the second part, we perform the perturbations provided by the first part at different places in such a way that the derivative of every (wandering or nonperiodic) orbit will be changed in the desirable way. For the (UD) property on the wandering set, the existence of open sets disjoint from all its iterates are very helpful, allowing us to spread the perturbation out over time. For the (LD) property, we need to control every non-periodic orbit. The existence of topological towers with very large return time, constructed in [BC], are the main tool, allowing us again to spread the perturbations out over a long time interval.

\section{Acknowledgments}

This paper grew out of several visits between the authors hosted by their home institutions. The authors would like to thank the Institut de Mathématiques de Bourgogne, the Northwestern University Math Department, and the Institut Galilée of the Université Paris 13 for their hospitality and support. This work was supported by NSF grants DMS-0401326 and DMS-0701018. We also thank Andrés Navas, who initially called our attention to the Lipschitz centralizer, and who pointed out several references to us.

\section{The local and global strategies}

In the remaining six sections, we prove the Main Theorem, following the outline in the Introduction. In this section, we reduce the proof to two results, Theorems A and $\mathrm{B}$, that together give a dense set of diffeomorphisms with the (UD) and (LD) properties.

\subsection{Background on $C^{1}$-generic dynamics}

The space $\operatorname{Diff}^{1}(M)$ is a Baire space in the $C^{1}$ topology. A residual subset of a Baire space is one that contains a countable intersection of open-dense sets; the Baire category theorem implies that a residual set is dense. We say that a property holds for the $C^{1}$-generic diffeomorphism if it holds on a residual subset of $\operatorname{Diff}^{1}(M)$.

For example, the Kupka-Smale Theorem asserts (in part) that for a $C^{1}$-generic diffeomorphism $f$, the periodic orbits of $f$ are all hyperbolic. It is easy to verify that, furthermore, the $C^{1}$-generic diffeomorphism $f$ has the following property: if $x, y$ are periodic points of $f$ with period $m$ and $n$ respectively, and if their orbits are distinct, then the set of eigenvalues of $D f^{m}(x)$ and of $D f^{n}(y)$ are disjoint. If this property holds, we say that the periodic orbits of $f$ have distinct eigenvalues.

Associated to any homeomorphism $f$ of a compact metric space $X$ are several canonically-defined, invariant compact subsets that contain the points in $X$ that are recurrent, to varying degrees, under $f$. Here we will use three of these sets, which are the closure of the periodic orbits, denoted here by $\overline{\operatorname{Per}(f)}$, the nonwandering set $\Omega(f)$, and the chain recurrent set $C R(f)$. By the canonical nature of their construction, the sets $\overline{\operatorname{Per}(f)}, \Omega(f)$ and $C R(f)$ are all preserved by any homeomorphism $g$ that commutes with $f$. 
We recall their definitions. The nonwandering set $\Omega(f)$ is the set of all points $x$ such that every neighborhood $U$ of $x$ meets some iterate of $U$ :

$$
U \cap \bigcup_{k>0} f^{k}(U) \neq \emptyset
$$

The chain recurrent set $C R(f)$ is the set of chain recurrent points defined as follows. Given $\varepsilon>0$, we say that a point $x$ is $\varepsilon$-recurrent, and write $x \sim_{\varepsilon} x$, if there exists an $\varepsilon$-pseudo-orbit, that is a sequence of points $x_{0}, x_{1}, \ldots, x_{k}, k \geq 1$ satisfying $d\left(f\left(x_{i}\right), x_{i+1}\right)<\varepsilon$, for $i=0, \ldots, k-1$, such that $x_{0}=x_{k}=x$. Then $x$ is chain recurrent if $x \sim_{\varepsilon} x$, for all $\varepsilon>0$. Conley theory implies that the complement of $C R(f)$ is the union of sets of the form $U \backslash \overline{f(U)}$ where $U$ is an open set which is attracting: $\overline{f(U)} \subset U$. The chain-recurrent set is partitioned into compact invariant sets called the chain-recurrence classes: two points $x, y \in C R(f)$ belong to the same class if one can join $x$ to $y$ and $y$ to $x$ by $\varepsilon$-pseudo-orbits for every $\varepsilon>0$.

It is not difficult to see that for any $f$, the inclusions $\overline{\operatorname{Per}(f)} \subseteq \Omega(f) \subseteq C R(f)$ hold; there exist examples where the inclusions are strict. For $C^{1}$ generic diffeomorphisms $f$, however, all three sets coincide; $\overline{\operatorname{Per}(f)}=\Omega(f)$ is a consequence of Pugh's closing lemma $[\mathrm{Pu}$, and $\Omega(f)=C R(f)$ was shown much more recently in [BC].

We have additional links between $\Omega(f)$ and the periodic points in the case it has non-empty interior:

Theorem $([\mathrm{BC}])$. For any diffeomorphism $f$ in a residual subset of $\operatorname{Diff}^{1}(M)$, any connected component $O$ of the interior of $\Omega(f)$ is contained in the closure of the stable manifold of a periodic point $p \in O$.

Conceptually, this result means that for $C^{1}$ generic $f$, the interior of $\Omega(f)$ and the wandering set $M \backslash \Omega(f)$ share certain nonrecurrent features. While points in the interior of $\Omega(f)$ all have nonwandering dynamics, if one instead considers the restriction of $f$ to a stable manifold of a periodic orbit $W^{s}(p) \backslash \mathcal{O}(p)$, the dynamics are no longer recurrent; in the induced topology on the submanifold $W^{s}(p) \backslash \mathcal{O}(p)$, every point has a wandering neighborhood $V$ whose iterates are all disjoint from $V$. Furthermore, the sufficiently large future iterates of such a wandering neighborhood are contained in a neighborhood of the periodic orbit. While the forward dynamics on the wandering set are not similarly "localized" as they are on a stable manifold, they still share this first feature: on the wandering set, every point has a wandering neighborhood (this time the neighborhood is in the topology on $M$ ).

Thus, the results in $\mathrm{BC}$ imply that for the $C^{1}$ generic $f$, we have the following picture: there is an $f$-invariant open and dense subset $W$ of $M$, consisting of the union of the interior of $\Omega(f)$ and the complement of $\Omega(f)$, and densely in $W$ the dynamics of $f$ can be decomposed into components with "wandering strata." We exploit this fact in our local strategy, outlined in the next section.

\subsection{Conditions for the local strategy: the unbounded distortion (UD) properties}

In the local strategy, we control the dynamics of the $C^{1}$ generic $f$ on the open and dense set $W=\operatorname{Int}(\Omega(f)) \cup(M \backslash \Omega(f))$. We describe here the main analytic properties we use to control these dynamics. 
We say that diffeomorphism $f$ satisfies the unbounded distortion property on the wandering set $\left(U D^{M \backslash \Omega}\right)$ if there exists a dense subset $\mathcal{X} \subset M \backslash \Omega(f)$ such that, for any $K>0$, any $x \in \mathcal{X}$ and any $y \in M \backslash \Omega(f)$ not in the orbit of $x$, there exists $n \geq 1$ such that:

$$
|\log | \operatorname{det} D f^{n}(x)|-\log | \operatorname{det} D f^{n}(y)||>K .
$$

A diffeomorphism $f$ satisfies the unbounded distortion property on the stable manifolds $\left(U D^{s}\right)$ if for any hyperbolic periodic orbit $\mathcal{O}$, there exists a dense subset $\mathcal{X} \subset W^{s}(\mathcal{O})$ such that, for any $K>0$, any $x \in \mathcal{X}$ and any $y \in W^{s}(\mathcal{O})$ not in the orbit of $x$, there exists $n \geq 1$ such that:

$$
|\log | \operatorname{det} D f_{\mid W^{s}(\mathcal{O})}^{n}(x)|-\log | \operatorname{det} D f_{\mid W^{s}(\mathcal{O})}^{n}(y)||>K .
$$

Our first main perturbation result is:

Theorem A (Unbounded distortion). The diffeomorphisms in a residual subset of $\operatorname{Diff}^{1}(M)$ satisfy the $\left(U D^{M \backslash \Omega}\right)$ and the $\left(U D^{s}\right)$ properties.

A variation of an argument due to Togawa [To1, To2] detailed in [BCW1] shows the $\left(\mathrm{UD}^{s}\right)$ property holds for a $C^{1}$-generic diffeomorphism. To prove Theorem A, we are thus left to prove that the $\left(\mathrm{UD}^{M \backslash \Omega}\right)$ property holds for a $C^{1}$-generic diffeomorphism. This property is significantly more difficult to establish $C^{1}$-generically than the $\left(\mathrm{UD}^{s}\right)$ property. The reason is that points on the stable manifold of a periodic point all have the same future dynamics, and these dynamics are "constant" for all large iterates: in a neighborhood of the periodic orbit, the dynamics of $f$ are effectively linear. In the wandering set, by contrast, the orbits of distinct points can be completely unrelated after sufficiently many iterates.

Nonetheless, the proofs that the $\left(\mathrm{UD}^{M \backslash \Omega}\right)$ and $\left(\mathrm{UD}^{s}\right)$ properties are $C^{1}$ residual share some essential features, and both rely on the essentially non-recurrent aspects of the dynamics on both the wandering set and the stable manifolds.

\subsection{Condition for the global strategy: the large derivative (LD) property}

Here we describe the analytic condition on the $C^{1}$-generic $f$ we use to extend the local conclusion on the centralizer of $f$ to a global conclusion.

A diffeomorphism $f$ satisfies the large derivative property (LD) on a set $X$ if, for any $K>0$, there exists $n(K) \geq 1$ such that for any $x \in X$ and $n \geq n(K)$, there exists $j \in \mathbb{Z}$ such that:

$$
\sup \left\{\left\|D f^{n}\left(f^{j}(x)\right)\right\|,\left\|D f^{-n}\left(f^{j+n}(x)\right)\right\|\right\}>K
$$

more compactly:

$$
\lim _{n \rightarrow \infty} \inf _{x \in X} \sup _{y \in \operatorname{orb}(x)}\left\{D f^{n}(y), D f^{-n}(y)\right\}=\infty .
$$

Rephrased informally, the (LD) property on $X$ means that the derivative $D f^{n}$ "tends to $\infty$ " uniformly on all orbits passing through $X$. We emphasize that the large 
derivative property is a property of the orbits of points in $X$, and if it holds for $X$, it also holds for all iterates of $X$.

Our second main perturbation result is:

Theorem B (Large derivative). Let $f$ be a diffeomorphism whose periodic orbits are hyperbolic. Then, there exists a diffeomorphism $g$ arbitrarily close to $f$ in $\operatorname{Diff}^{1}(M)$ such that the property (LD) is satisfied on $M \backslash \operatorname{Per}(f)$.

Moreover,

- $f$ and $g$ are conjugate via a homeomorphism $\Phi$, i.e. $g=\Phi f \Phi^{-1}$;

- for any periodic orbit $\mathcal{O}$ of $f$, the derivatives of $f$ on $\mathcal{O}$ and of $g$ on $\Phi(\mathcal{O})$ are conjugate (in particular the periodic orbits of $g$ are hyperbolic);

- if $f$ satisfies the $\left(U D^{M \backslash \Omega}\right)$ property, then so does $g$;

- if $f$ satisfies the $\left(U D^{s}\right)$ property, then so does $g$.

As a consequence of Theorems A and B we obtain:

Corollary 2.1. There exists a dense subset $\mathcal{D}$ of $\operatorname{Diff}^{1}(M)$ such that any $f \in \mathcal{D}$ satisfies the following properties:

- the periodic orbits are hyperbolic and have distinct eigenvalues;

- any component $O$ of the interior of $\Omega(f)$ contains a periodic point whose stable manifold is dense in $O$;

- $f$ has the $\left(U D^{M \backslash \Omega}\right)$ and the $\left(U D^{s}\right)$ properties;

- $f$ has the (LD) property on $M \backslash \operatorname{Per}(g)$.

\subsection{Checking that the centralizer is trivial}

We now explain why properties (UD) and (LD) together imply that the centralizer is trivial.

Proposition 2.2. Any diffeomorphism $f$ in the $C^{1}$-dense subset $\mathcal{D} \subset \operatorname{Diff}^{1}(M)$ given by Corollary 2.1 has a trivial centralizer $Z^{1}(f)$.

Proof of Proposition 2.2. Consider a diffeomorphism $f \in \mathcal{D}$. Let $g \in Z^{1}(f)$ be a diffeomorphism commuting with $f$, and let $K>0$ be a Lipschitz constant for $g$ and $g^{-1}$. Let $W=\operatorname{Int}(\Omega(f)) \cup(M \backslash \Omega(f))$ be the $f$-invariant, open and dense subset of $M$ whose properties are discussed in Section 2.1.

Our first step is to use the "local hypotheses" (UD $\left.{ }^{M \backslash \Omega}\right)$ and $\left(\mathrm{UD}^{s}\right)$ to construct a function $\alpha: W \rightarrow \mathbb{Z}$ that is constant on each connected component of $W$ and satisfies $g=f^{\alpha}$. We then use the "global hypothesis" (LD) to show that $\alpha$ is bounded on $W$, and therefore extends to a constant function on $M$. 
We first contruct $\alpha$ on the wandering set $M \backslash \Omega(f)$. The basic properties of Lipschitz functions and the relation $f^{n} g=g f^{n}$ imply that for any $x \in M$, and any $n \in \mathbb{Z}$, we have

$$
\left|\log \operatorname{det}\left(D f^{n}(x)\right)-\log \operatorname{det}\left(D f^{n}(g(x))\right)\right| \leq 2 d \log K
$$

where $d=\operatorname{dim} M$. On the other hand, $f$ satisfies the $\mathrm{UD}^{M \backslash \Omega(f)}$ property, and hence there exists a dense subset $\mathcal{X} \subset M \backslash \Omega(f)$, each of whose points has unbounded distortion with respect to any point in the wandering set not on the same orbit. That is, for any $x \in \mathcal{X}$, and $y \in M \backslash \Omega(f)$ not on the orbit of $x$, we have:

$$
\limsup _{n \rightarrow \infty}|\log | \operatorname{det} D f^{n}(x)|-\log | \operatorname{det} D f^{n}(y)||=\infty .
$$

Inequality (11) then implies that $x$ and $y=g(x)$ lie on the same orbit, for all $x \in \mathcal{X}$, hence $g(x)=f^{\alpha(x)}(x)$. Using the continuity of $g$ and the fact that the points in $M \backslash \Omega(f)$ admit wandering neighborhoods whose $f$-iterates are pairwise disjoint, we deduce that the map $\alpha: \mathcal{X} \rightarrow \mathbb{Z}$ is constant in the neighborhood of any point in $M \backslash \Omega(f)$. Hence the function $\alpha$ extends on $M \backslash \Omega(f)$ to a function that is constant on each connected component of $M \backslash \Omega(f)$. Furthermore, $g=f^{\alpha}$ on $M \backslash \Omega(f)$.

We now define the function $\alpha$ on the interior $\operatorname{Int}(\Omega(f))$ of the nonwandering set. Since the periodic orbits of $f \in \mathcal{D}$ have distinct eigenvalues and since $g$ preserves the rate of convergence along the stable manifolds, the diffeomorphism $g$ preserves each periodic orbit of $f$. Using the $\left(U D^{s}\right)$ condition, one can extend the argument above for the wandering set to the stable manifolds of each periodic orbit (see also BCW1, Lemma 1.2]). We obtain that for any periodic point $p$, the diffeomorphism $g$ coincides with a power $f^{\alpha}$ on each connected component of $W^{s}(p) \backslash\{p\}$. For $f \in \mathcal{D}$, each connected component $O$ of the interior of $\Omega(f)$ contains a periodic point $p$ whose stable manifold is dense in $O$. It follows that $g$ coincides with some power $f^{\alpha}$ of $f$ on each connected component of the interior of $\Omega(f)$.

We have seen that there is a locally constant function $\alpha: W \rightarrow \mathbb{Z}$ such that $g=f^{\alpha}$ on the $f$ invariant, open and dense subset $W \subset M$. We now turn to the global strategy. Notice that, since $f$ and $g$ commute, the function $\alpha$ is constant along the non-periodic orbits of $f$. Now $f \in \mathcal{D}$ satisfies the (LD) property. Consequently there exists $N>0$ such that, for every non-periodic point $x$, and for every $n \geq N$ there is a point $y=f^{i}(x)$ such that either $\left\|D f^{n}(y)\right\|>K$ or $\left\|D f^{-n}(y)\right\|>K$. This implies that the function $|\alpha|$ is bounded by $N$ : otherwise, $\alpha$ would be greater than $N$ on the invariant open set $W$ of $M$. This open set contains a non-periodic point $x$ and an iterate $y=f^{i}(x)$ such that either $\left\|D f^{\alpha}(y)\right\|>K$ or $\left\|D f^{-\alpha}(y)\right\|>K$. This contradicts the fact that $g$ and $g^{-1}$ are $K$-Lipschitz.

We have just shown that $|\alpha|$ is bounded by some integer $N$. Let $\operatorname{Per}_{2 N}$ be the set of periodic points of $f$ whose period is less than $2 N$, and for $i \in\{-N, \ldots, N\}$ consider the set

$$
P_{i}=\left\{x \in M \backslash \operatorname{Per}_{2 N}, g(x)=f^{i}(x)\right\} .
$$

This is a closed invariant subset of $M \backslash \operatorname{Per}_{2 N}$. What we proved above implies that $M \backslash \operatorname{Per}_{2 N}$ is the union of the sets $P_{i},|i| \leq N$. Moreover any two sets $P_{i}, P_{j}$ with $i \neq j$ are disjoint since a point in $P_{i} \cap P_{j}$ would be $|i-j|$ periodic for $f$. 
If $\operatorname{dim}(M) \geq 2$, since $M$ is connected and $\operatorname{Per}_{2 N}$ is finite, the set $M \backslash \operatorname{Per}_{2 N}$ is connected. It follows that only one set $P_{i}$ is non-empty, implying that $g=f^{i}$ on $M$. This concludes the proof in this case.

If $\operatorname{dim}(M)=1$, one has to use that $g$ is a diffeomorphism and is not only Lipschitz: this shows that on the two sides of a periodic orbit of $f$, the map $g$ coincides with the same iterate of $f$. This proves again that only one set $P_{i}$ is nonempty.

\subsection{From dense to residual: compactness and semicontinuity}

The previous results show that the set of diffeomorphisms having a trivial centralizer is dense in $\operatorname{Diff}^{1}(M)$, but this is not enough to conclude the proof of the Main Theorem. Indeed the dense subset $\mathcal{D}$ in Corollary 2.1 is not a residual subset if $\operatorname{dim}(M) \geq 2$ : in the appendix we exhibit a nonempty open set in which $C^{1}$-generic diffeomorphisms do not satisfy the (LD)-property.

Fix a metric structure on $M$. A homeomorphism $f: M \rightarrow M$ is $K$-bi-Lipschitz if both $f$ and $f^{-1}$ are Lipschitz, with Lipschitz norm bounded by $K$. A homeomorphism that is $K$-bi-Lipschitz for some $K$ is called a bi-Lipschitz homeomorphism, or lipeomorphism. We denote by $\operatorname{Lip}^{K}(M)$ the set of $K$-bi-Lipschitz homeomorphisms of $M$ and by $\operatorname{Lip}(M)$ the set of bi-Lipschitz homeomorphisms of $M$. The ArzèlaAscoli theorem implies that $\operatorname{Lip}^{K}(M)$ is compact in the uniform $\left(C^{0}\right)$ topology. Note that $\operatorname{Lip}(M) \supset \operatorname{Diff}^{1}(M)$. For $f \in \operatorname{Lip}(M)$, the set $Z^{\operatorname{Lip}}(f)$ is defined analogously to the $C^{r}$ case:

$$
Z^{\operatorname{Lip}}(f):=\{g \in \operatorname{Lip}(M): f g=g f\} .
$$

In dimension 1, the Main Theorem was a consequence of Togawa's work [To2]. In higher dimension, the Main Theorem is a direct corollary of:

Theorem 2.3. If $\operatorname{dim}(M) \geq 2$, the set of diffeomorphisms $f$ with trivial centralizer $Z^{\mathrm{Lip}}(f)$ is residual in $\operatorname{Diff}^{1}(M)$.

The proof of Theorem 2.3 has two parts.

Proposition 2.4. If $\operatorname{dim}(M) \geq 2$, any diffeomorphism $f$ in the $C^{1}$-dense subset $\mathcal{D} \subset \operatorname{Diff}^{1}(M)$ given by Corollary 2.1 has a trivial centralizer $Z^{\mathrm{Lip}}(f)$.

The proof of this proposition from Theorems A and B is the same as the proof of Proposition 2.2 (see also Lemma 1.2 in [BCW1]).

Proposition 2.5. Consider the set $\mathcal{T}$ of diffeomorphisms $f \in \operatorname{Diff}^{1}(M)$ having a trivial centralizer $Z^{\operatorname{Lip}}(f)$. Then, if $\mathcal{T}$ is dense in $\operatorname{Diff}^{1}(M)$, it is also residual.

Remark 2.6. The proof of Proposition 2.5 also holds in the $C^{r}$ topology $r \geq 2$ on any manifold $M$ on which the $C^{r}$-generic diffeomorphism has at least one hyperbolic periodic orbit (for example, on the circle, or on manifolds of nonzero Euler characteristic). On the other hand, Theorem 2.3 is false for general manifolds in the $C^{2}$ topology, at least for the circle. In fact, a simple folklore argument (see the proof of Theorem B in [N]) implies that for any Kupka-Smale diffeomorphism $f \in \operatorname{Diff}^{2}\left(S^{1}\right)$, the set $Z^{\operatorname{Lip}}(f)$ is infinite dimensional. It would be interesting to find out what is true in higher dimensions. 
Proof of Proposition 2.5. For any compact metric space $X$ we denote by $\mathcal{K}(X)$ the set of non-empty compact subsets of $X$, endowed with the Hausdorff distance $d_{H}$. We use the following classical fact.

Proposition. Let $\mathcal{B}$ be a Baire space, let $X$ be a compact metric space, and let $h: \mathcal{B} \rightarrow \mathcal{K}(X)$ be an upper-semicontinuous function. Then the set of continuity points of $h$ is a residual subset of $\mathcal{B}$.

In other words, if $h$ has the property that for all $b \in \mathcal{B}$,

$$
b_{n} \rightarrow b \Longrightarrow \limsup b_{n}=\bigcap_{n} \overline{\bigcup_{i>n} h\left(b_{i}\right)} \subseteq h(b),
$$

then there is a residual set $\mathcal{R}_{h} \subset \mathcal{B}$ such that, for all $b \in \mathcal{R}_{h}$,

$$
b_{n} \rightarrow b \Longrightarrow \lim d_{H}\left(b_{n}, b\right)=0 .
$$

To prove Proposition 2.5, we note that for a fixed $K>0$, the set $Z^{\operatorname{Lip}}(f) \cap$ $\operatorname{Lip}^{K}(M)$ is a closed subset (in the $C^{0}$ topology) of the compact metric space $\operatorname{Lip}^{K}(M)$. This is a simple consequence of the facts that $Z^{\operatorname{Lip}}(f)$ is defined by the relation $f g f^{-1} g^{-1}=\mathrm{id}$, and that composition and inversion are continuous. Thus there is well-defined map $h_{K}$ from $\operatorname{Diff}^{1}(M)$ to $\mathcal{K}\left(\operatorname{Lip}^{K}(M)\right)$, sending $f$ to $h_{K}(f)=Z^{\operatorname{Lip}}(f) \cap \operatorname{Lip}^{K}(M)$. It is easy to see that $h_{K}$ is upper-semicontinuous: if $f_{n}$ converges to $f$ in $\operatorname{Diff}^{1}(M)$ and $g_{n} \in h_{K}\left(f_{n}\right)$ converges uniformly to $g$ then $g$ belongs to $h_{K}(f)$.

Let $\mathcal{R}_{K} \subset \operatorname{Diff}^{1}(M)$ be the set of points of continuity of $h_{K}$; it is a residual subset of $\operatorname{Diff}^{1}(M)$, by Proposition 2.5. Let $\mathcal{R}_{H y p} \subset \operatorname{Diff}^{1}(M)$ be the set of diffeomorphisms such that each $f \in \mathcal{R}_{\text {Hyp }}$ has at least one hyperbolic periodic orbit (the $C^{1}$ Closing Lemma implies that $\mathcal{R}_{\text {Hyp }}$ is residual). Finally, let

$$
\mathcal{R}=\mathcal{R}_{H y p} \cap \bigcap_{K=1}^{\infty} \mathcal{R}_{K} .
$$

Assuming that $\mathcal{T}$ is dense in $\operatorname{Diff}^{1}(M)$, we claim that the set $\mathcal{R}$ is contained in $\mathcal{T}$, implying that $\mathcal{T}$ is residual. To see this, fix $f \in \mathcal{R}$, and let $f_{n} \rightarrow f$ be a sequence of diffeomorphisms in $\mathcal{T}$ converging to $f$ in the $C^{1}$ topology. Let $g \in Z^{\operatorname{Lip}}(M)$ be a $K$-bi-Lipschitz homeomorphism satisfying $f g=g f$. Since $h_{K}$ is continuous at $f$, there is a sequence $g_{n} \in Z^{\mathrm{Lip}}\left(f_{n}\right)$ of $K$-bi-Lipschitz homeomorphisms with $g_{n} \rightarrow g$ in the $C^{0}$ topology. The fact that $f_{n} \in \mathcal{T}$ implies that the centralizer $Z^{\operatorname{Lip}}\left(f_{n}\right)$ is trivial, so there exist integers $m_{n}$ such that $g_{n}=f^{m_{n}}$.

If the sequence $\left(m_{n}\right)$ is bounded, then passing to a subsequence, we obtain that $g=f^{m}$, for some integer $m$. If the sequence $\left(m_{n}\right)$ is not bounded, then we obtain a contradiction as follows. Let $x$ be a hyperbolic periodic point of $f$, of period $p$. For $n$ large, the map $f_{n}$ has a periodic orbit $x_{n}$ of period $p$, and the derivatives $D f_{n}^{p}\left(x_{n}\right)$ tend to the derivative $D f^{p}(x)$. But then $\left|\log \left\|D f_{n}^{m_{n}}\right\|\right|$ tends to infinity as $n \rightarrow \infty$. This contradicts the fact that the diffeomorphisms $f_{n}^{m_{n}}=g_{n}$ and $f_{n}^{-m_{n}}=g_{n}^{-1}$ are both $K$-Lipschitz, concluding the proof. 


\section{Perturbing the derivative without changing the dy- namics}

In order to prove Theorem $\mathrm{B}$, one needs to perturb a diffeomorphism $f$ and change the dynamical properties of its derivative without changing its topological dynamics: the resulting diffeomorphism is still conjugate to $f$. We develop in this section an important technique for the proof, which we call tidy perturbations of the dynamics.

\subsection{Tidy perturbations}

Definition 3.1. Let $f: M \rightarrow M$ be a homeomorphism and let $X \subset M$. We say that a homeomorphism $g$ is a tidy perturbation of $f$ supported on $X$ if:

1. $g(x)=f(x)$, for all $x \in M \backslash X$,

2. if $x \in M \backslash X$ and $f^{m}(x) \in M \backslash X$, for some $m \geq 1$, then $g^{m}(x)=f^{m}(x)$;

3. if $x \in M \backslash X$ and $g^{m}(x) \in M \backslash X$, for some $m \geq 1$, then $g^{m}(x)=f^{m}(x)$;

4. for all $x \in M$, there exists $m \in \mathbb{Z}$ such that $f^{m}(x) \in M \backslash \bar{X}$;

5. for all $x \in M$, there exists $m \in \mathbb{Z}$ such that $g^{m}(x) \in M \backslash \bar{X}$.

Note that this definition is symmetric in $f$ and $g$.

Lemma 3.2. If $g$ is a tidy perturbation of $f$ supported on $X$, then $g$ is conjugate to $f$ by a homeomorphism $\varphi$ such that $\varphi=\mathrm{id}$ on $M \backslash(X \cap f(X))$. Furthermore, if $g$ and $f$ are diffeomorphisms, then $\varphi$ is a diffeomorphism as well.

Proof. Given $f$ and a tidy perturbation $g$ of $f$ supported on $X$, we construct a homeomorphism $\varphi$ as follows. Property 4 of tidy perturbations implies that for each $x \in M$, there exists an integer $m_{x}$ such that $f^{m_{x}}(x) \in M \backslash \bar{X}$. We set $\varphi(x)=$ $g^{-m_{x}} f^{m_{x}}(x)$.

Then $\varphi$ is well-defined, for suppose that $f^{m_{1}}(x) \notin \bar{X}$ and $f^{m_{2}}(x) \notin \bar{X}$, for some integers $m_{1}<m_{2}$. Let $y=f^{m_{1}}(x)$. Then $y \notin \bar{X}$, and $f^{m_{2}-m_{1}}(y)=f^{m_{2}}(x) \notin \bar{X}$. Property 2 of tidy perturbations implies that $g^{m_{2}-m_{1}}(y)=f^{m_{2}-m_{1}}(y)$; in other words, $g^{-m_{1}} f^{m_{1}}(x)=g^{-m_{2}} f^{m_{2}}(x)$. Hence the definition of $\varphi(x)$ is independent of the integer $m_{x}$. In particular $g \circ \varphi=\varphi \circ f$.

To see that $\varphi$ is continuous, note that for every $x \in M$, if $f^{m}(x) \notin \bar{X}$, then there exists a neighborhood $U$ of of $x$ such that $f^{m}(U) \cap \bar{X} \neq \emptyset$. Hence $\varphi=g^{-m} \circ f^{m}$ on $U$. This implies that $\varphi$ is a local homeomorphism.

Let $\varphi^{-}$be the local homeomorphism obtained by switching the roles of $f$ and $g$. Clearly $\varphi^{-}$is the inverse of $\varphi$, so that $\varphi$ is a homeomorphism. If $f$ and $g$ are diffeomorphisms, it is clear from the construction of $\varphi$ that $\varphi$ is a diffeomorphism.

Properties 1 and 2 of tidy perturbations imply that for any point $x \in M \backslash(X \cap$ $f(X))$, we have $f^{m_{x}}(x)=g^{m_{x}}(x)$. This gives $\varphi=$ id on $M \backslash(X \cap f(X))$.

Each the tidy perturbations we will consider is supported in the union of succesive disjoint iterates of an open set. In that case, the characterization of tidy perturbations is much easier, as explained in the next lemma: 
Lemma 3.3. Let $f$ be a homeomorphism, $U \subset M$ an open set, and $m \geq 1$ an integer such that the iterates $\bar{U}, f(\bar{U}), \ldots, f^{m}(\bar{U})$ are pairwise disjoint.

Let $g$ be a homeomorphism such that:

- $g=f$ on the complement of the union $X=\bigcup_{i=0}^{m-1} f^{i}(U)$, and

- for all $x \in U, f^{m}(x)=g^{m}(x)$.

Then $g$ is a tidy perturbation of $f$ supported on $X$.

Proof. Property 1 of tidy perturbations is immediate; property 4 is as well, since $\bar{U}, f(\bar{U}), \ldots, f^{m}(\bar{U})$ are pairwise disjoint.

Let $x$ be a point in $M \backslash X$ whose $f$-orbit enters and leaves $X$, and let $i>0$ be its first entry in $X$; then $f^{i}(x)=g^{i}(x)$ by the first hypothesis on $g$. The $f$-orbit of $x$ leaves $X$ at $f^{m+i}(x)=g^{m+i}(x)$, by the second hypothesis on $g$. Proceeding inductively on the successive entrances and exits of the $f$-orbit of $x$ we get that $g^{n}(x)=f^{n}(x)$ for every integer $n$ such that $f^{n}(x) \in M \backslash X$, proving Property 2 of tidy perturbations.

There is a neighborhood $V$ of $\bar{U}$ such that $f=g$ on $V \backslash U$. It follows that $f(U)=g(U)$. Inductively, we see that $g^{i}(U)=f^{i}(U)$ for $i \in\{1, \ldots, m\}$. This shows that the hypotheses of the lemma still are satisfied if we switch the roles of $f$ and g. This implies Properties 3 and 5 of tidy perturbations.

If $g$ is a small $C^{1}$-perturbation of $f$ that is a tidy perturbation supported in the disjoint union $\bigcup_{i=0}^{m-1} f^{i}(U)$, it is tempting to think that the conjugating diffeomorphism $\varphi$ must be $C^{1}$-close to the identity as well. This is not always the case. The derivative $D g$ is a small perturbation of $D f$ but for $x \in U$ and $i \in\{1, \ldots, m\}$, the maps $D f^{i}(x)$ and $D g^{i}(x)$ could be very different. There is however a straighforward a priori bound for $D \varphi$ :

Lemma 3.4. Let $f$ be a diffeomorphism, $U \subset M$ an open set and $m \geq 1$ an integer such that the iterates $\bar{U}, f(\bar{U}), \ldots, f^{m}(U)$ are pairwise disjoint. Suppose that $g$ is a tidy perturbation of $f$ supported on $X=\bigcup_{i=0}^{m-1} f^{i}(\bar{U})$ and that $\varphi: M \rightarrow M$ is the diffeomorphism such that $g=\varphi \circ f \circ \varphi^{-1}$ and $\varphi=$ id on $M \backslash X$.

Then

$$
\max \left\{\|D \varphi\|,\left\|D \varphi^{-1}\right\|\right\} \leq C^{m},
$$

where $C=\sup \left\{\|D g\|,\left\|D g^{-1}\right\|,\|D f\|,\left\|D f^{-1}\right\|\right\}$.

Proof. For every $x \in M$ there exists $i \in\{0, \ldots, m\}$ such that $f^{i}(x) \notin \bar{X}$, so that $\varphi$ coincides with $g^{-i} f^{i}$ and $g^{-i+m} f^{i-m}$ in a neighborhood of $x$. We conclude by noting that either $2 i$ or $2(m-i)$ is less than $m$.

\subsection{Sequences of tidy perturbations}

The aim of this section is to control the effect of infinitely many successive tidy perturbations $g_{i}$ of a diffeomorphism $f$ and to give a sufficient condition for the sequence $g_{i}$ to converge to a diffeomorphism $g$ conjugate to $f$ by a homeomorphism. 
Lemma 3.5. Let $f$ be a diffeomorphism and $\varepsilon, C>0$ be constants such that for every $g$ with $d_{C^{1}}(f, g)<\varepsilon$, we have $\|D g\|,\left\|D g^{-1}\right\|<C$. Consider:

- $\left(U_{i}\right)_{i \geq 1}$, a sequence of open subsets of $M$,

- $\left(m_{i}\right)_{i \geq 1}$, a sequence of positive integers,

- $\left(\varepsilon_{i}\right)_{i \geq 1}$, a sequence of positive numbers such that $\sum \varepsilon_{i}<\varepsilon$,

- $\left(g_{i}\right)_{i \geq 0}$, a sequence of diffeomorphisms such that $g_{0}=f$ and for each $i \geq 1$,

- the sets $\overline{U_{i}}, g_{i-1}\left(\overline{U_{i}}\right), \ldots, g_{i-1}^{m_{i}}\left(\overline{U_{i}}\right)$ are pairwise disjoint,

- $g_{i}$ is a tidy $\varepsilon_{i}$-perturbation of $g_{i-1}$, supported in $\bigcup_{k=0}^{m_{i}-1} g_{i-1}^{k}\left(U_{i}\right)$,

- $\left(\rho_{i}\right)_{i \geq 1}$, a sequence of positive numbers such that $g_{i-1}^{k}\left(U_{i}\right)$ has diameter bounded by $\rho_{i}$, for $k \in\left\{0, \ldots, m_{i}\right\}$.

Denote by $\varphi_{i}$ the diffeomorphism such that $g_{i}=\varphi_{i} \circ g_{i-1} \circ \varphi_{i}^{-1}$ and $\varphi_{i}=\mathrm{id}$ on $M \backslash \bigcup_{k=1}^{m_{i}-1} g_{i-1}^{k}\left(U_{i}\right)$. Let $\Phi_{i}=\varphi_{i} \circ \cdots \circ \varphi_{1}$ and $M_{i}=\Pi_{k=1}^{i} C^{m_{k}}$. If one assumes that

$$
\sum_{i \geq 1} \rho_{i} M_{i-1}<\infty
$$

then

1. $\left(g_{i}\right)$ converges in the $C^{1}$ metric to a diffeomorphism $g$ with $d_{C^{1}}(f, g)<\varepsilon$,

2. for all $i<j$ one has $d_{\text {unif }}\left(\Phi_{j} \Phi_{i}^{-1}, \mathrm{id}\right)<\sum_{k=i+1}^{j} \rho_{k}$,

3. $\left(\Phi_{i}\right)$ converges uniformly to a homeomorphism $\Phi$ satisfying $g=\Phi f \Phi^{-1}$.

Remark 3.6. The uniform metric $d_{\text {unif }}$ on continuous self-maps on $M$ in part 3 of this lemma is not complete for the space of homeomorphisms of $M$; to avoid confusion, we denote by $d_{C^{0}}$ the complete metric defined by:

$$
d_{C^{0}}(f, g)=d_{\text {unif }}(f, g)+d_{\text {unif }}\left(f^{-1}, g^{-1}\right) .
$$

One difficulty in the proof of Lemma 3.5, which explains the role of $M_{i}$, is to control the distances $d_{\text {unif }}\left(\Phi_{i}^{-1} \Phi_{j}, \mathrm{id}\right)$.

Proof. The first conclusion is clear. Since $\Phi_{j} \Phi_{i}^{-1}=\varphi_{j} \circ \cdots \circ \varphi_{i+1}$, the second one is immediate from the hypothesis that $\varphi_{i}$ is a tidy perturbation and the fact that the connected components of the support of $\varphi_{i}$ have diameter less than $\rho_{i}$. The hypothesis $\sum_{k \geq 1} \rho_{i}<\infty$ implies that $\left(\Phi_{i}\right)_{i \in \mathbb{N}}$ is a Cauchy sequence and converges to a continuous map $\Phi$ satisfying $g \Phi=\Phi f$. Finally, the a priori bound in Lemma 3.4 implies that $\left\|D \Phi_{i}^{-1}\right\|<M_{i}$, so that

$$
\sup _{d(x, y)<\rho_{i}} d\left(\Phi_{i-1}^{-1}(x), \Phi_{i-1}^{-1}(y)\right)<\rho_{i} M_{i-1} .
$$

But for any $x$, we have $d\left(x, \varphi_{i}(x)\right)<\rho_{i}$. So the previous calculation implies $d\left(\Phi_{i-1}^{-1}(x), \Phi_{i}^{-1}(x)\right)<\rho_{i} M_{i-1}$. By hypothesis, $\sum_{i \geq 1} \rho_{i} M_{i-1}<\infty$, which implies that $\left(\Phi_{i}^{-1}\right)_{i \in \mathbb{N}}$ is a Cauchy sequence in the $d_{\text {unif }}$ metric. Hence $\left(\Phi_{i}^{-1}\right)$ converges as $i \rightarrow \infty$ to the inverse of $\Phi$ and so $\Phi$ is a homeomorphism. 


\subsection{Topological towers}

For each tidy perturbation we construct in this paper, we will use an open set with many disjoint iterates. The large number of iterates will allow us to spread the effects of the perturbation out over a large number of steps, effecting a large change in the derivative with a small perturbation. For these perturbations to have a global effect, we need to have most orbits in $M$ visit $U$. The technique of topological towers, developed in $[\mathrm{BC}]$, allows us to choose $U$ to have many disjoint iterates, while simultaneously guaranteeing that most orbits visit $U$.

Theorem 3.7 (Topological towers, [BC], Théorème 3.1). For any integer $d \geq 1$, there exists a constant $\kappa_{d}>0$ such that for any integer $m \geq 1$ and for any diffeomorphism $f$ of a d-dimensional manifold $M$, whose periodic orbits of period less than $\kappa_{d} . m$ are hyperbolic, there exists an open set $U$ and a compact subset $D \subset U$ having the following properties:

- Any point $x \in M$ that does not belong to a periodic orbit of period $<m$ has an iterate $f^{i}(x)$ in the interior of $D$.

- The sets $\bar{U}, f(\bar{U}), \ldots, f^{m-1}(\bar{U})$ are pairwise disjoint.

Moreover, the connected components of $\bar{U}$ can be chosen with arbitrarily small diameter.

\subsection{Towers avoiding certain sets}

In constructing our sequence $\left(g_{i}\right)$ of tidy perturbations, we need to ensure that the effects of the $(i+1)$ st perturbations do not undo the effects of the $i$ th perturbation. In addition, we aim to produce large derivative without affecting unbounded distortion. For these reasons, it is desirable to choose the towers in the tidy perturbations to avoid certain subsets in $M$. It is not possible to choose a tower avoiding an arbitrary subset, but it turns out that certain sets with a wandering orbit property can be avoided. We now define this property.

Definition 3.8. Let $N, J \geq 1$ be integers. An $(N, J)$-wandering cover of a compact set $Z$ is a finite cover $\mathcal{U}$ of $Z$ such that every $V \in \mathcal{U}$ has $N$ iterates $f^{j}(V), f^{j+1}(V), \ldots, f^{j+N-1}(V)$ with $j \in\{1, \ldots, J\}$ that are disjoint from $Z$.

Definition 3.9. Let $f$ be a homeomorphism and $Z \subset M$ be a compact set.

$Z$ has the $(N, J)$-wandering orbit property if it has an $(N, J)$-wandering cover.

$Z$ has the $N$-wandering orbit property if it has the $(N, J)$-wandering orbit property for some $J \geq 1$.

$Z$ has the wandering orbit property it has the $N$-wandering orbit property, for every $N \geq 1$.

The next lemma explains that topological towers can be constructed avoiding any compact set with the wandering orbit property.

Lemma 3.10. Suppose that the periodic orbits of $f$ are all hyperbolic.

For all $m_{1}, m_{2} \geq 1$, and $\rho>0$, if $Z$ is any compact set with the $m_{1}$-wandering orbit property, then there exists an open set $U \subset M$ with the following properties: 
1. The diameter of each connected component of $\bar{U}$ is less than $\rho$.

2. The iterates $\bar{U}, f(\bar{U}), \ldots, f^{m_{1}-1}(\bar{U})$ are disjoint from the set $Z$.

3. The iterates $\bar{U}, f(\bar{U}), \ldots, f^{m_{1}+m_{2}-1}(\bar{U})$ are pairwise disjoint.

4. There is a compact set $D \subset U$ such that every nonperiodic point $x \in M$ has an iterate in $D$.

This will be proved at the end of this subsection.

Remark 3.11. - If $Z$ has the $(N, J)$-wandering orbit property, then for every $\rho>0$, there is an $(N, J)$-wandering cover all of whose elements have diameter less than $\rho$.

- If $Z$ has the $(N, J)$-wandering orbit property, then so does $f(Z)$.

- More generally, if $Z$ has the $(N, J)$-wandering orbit property for $f$, and $g=$ $\Phi f \Phi^{-1}$, then $\Phi(Z)$ has the $(N, J)$-wandering orbit property for $g$.

Lemma 3.12. 1 . If $X$ is any compact set such that the iterates $X, f(X), \ldots$, $f^{m+N}(X)$ are pairwise disjoint, then $\bigcup_{i=0}^{m-1} f^{i}(X)$ has the $(N, m)$-wandering orbit property.

2. If $x$ is any point whose period lies in $(N+1, \infty]$, then $\{x\}$ has the $(N, 1)$ wandering orbit property.

3. If $x$ is any nonperiodic point, then $\{x\}$ has the wandering orbit property.

4. Any compact subset $Z \subset \mathcal{W}^{s}(\operatorname{orb}(p)) \backslash \operatorname{orb}(p)$ has the wandering orbit property, for any hyperbolic periodic point $p$.

5. Any compact subset of the wandering set $M \backslash \Omega(f)$ of $f$ has the wandering orbit property.

Proof. 1), 2) and (3) are easy. To prove (4), we consider $Z \subset W^{s}(\operatorname{orb}(p)) \backslash \operatorname{orb}(p)$, for some hyperbolic periodic point $p$. Let $\mathcal{N}$ be a neighborhood of the orbit of $p$ that is disjoint from $Z$. There exists $m>0$ such that $f^{n}(Z) \subset \mathcal{N}$ for any $n \geq m$. Given $N$, there is a covering $\mathcal{V}$ of $Z$ such that, for every $V \in \mathcal{V}$, the sets $f^{m}(V), \ldots, f^{m+N}(V)$ are all contained in $\mathcal{N}$. Then $\mathcal{V}$ is an $(N, m)$-wandering cover of $Z$.

Finally we prove 5 ). If $Z$ is a compact subset of $M \backslash \Omega(f)$ then every point $z \in Z$ has finitely many returns in $Z$. So for every $N>0$ and every $x \in Z$ there is a neighborhood $U_{x}$ and an integer $j_{x}>0$ such that $f^{j_{x}}\left(U_{x}\right), \ldots, f^{j_{x}+N-1}\left(U_{x}\right)$ are disjoint from $Z$. Extracting a finite cover $U_{x_{i}}$ and chosing $J=\max _{i} j_{x_{i}}$ we get an $(N, J)$-wandering cover.

Lemma 3.13. Suppose $Z$ has the $(N, J)$-wandering orbit property and $W$ has the $(2 N+J, K)$-wandering orbit property. Then $Z \cup W$ has the $(N, K+2 J+2 N)$ wandering orbit property.

If $Z$ has the $N$-wandering orbit property and $W$ has the wandering orbit property, then $Z \cup W$ has the $N$-wandering orbit property.

If $Z$ and $W$ both have the wandering orbit property, then $Z \cup W$ has the wandering orbit property. 
Proof. The second two claims follow from the first. To prove the first, fix a $(N, J)$-wandering cover $\mathcal{U}_{Z}$ of $Z$ and a $(2 N+J, K)$-wandering cover $\mathcal{V}_{W}$ of $W$ with the property that, for every $V \in \mathcal{V}_{W}$ and every $i \in\{0, \ldots, N+K-1\}$, if $f^{i}(V)$ intersects $Z$, then $f^{i}(V)$ is contained in an element of $\mathcal{U}_{Z}$. Let $\mathcal{V}_{Z}$ be an $(N, J)$ wandering cover of $Z$ such that, for every $V \in \mathcal{V}_{Z}$ and every $i \in\{0, \ldots, N+J-1\}$, if $f^{i}(V)$ intersects $W$, then $f^{i}(V)$ is contained in an element of $\mathcal{V}_{W}$. We claim that $\mathcal{V}_{Z} \cup \mathcal{V}_{W}$ is an $(N, K+2 J+2 N)$-wandering cover of $Z \cup W$ :

- Consider first $V \in \mathcal{V}_{W}$. As $\mathcal{V}_{W}$ is a $(2 N+J, K)$-wandering cover of $W$, there exists $k \in\{1, \ldots, K\}$ such that $f^{k}(V), \ldots, f^{k+2 N+J-1}(V)$ are disjoint from $W$. If $f^{k}(V), \ldots, f^{k+N-1}(V)$ are disjoint from $Z$, then they are disjoint from $W \cup Z$ which is what we want. Otherwise, there exists $V^{\prime} \in \mathcal{U}_{Z}$ and $j \in\{1, \ldots, N-1\}$ such that $f^{k+j}(V) \subset V^{\prime}$. Now there exists $i \in\{1, \ldots, J\}$ such that $f^{i}\left(V^{\prime}\right), \ldots, f^{i+N-1}\left(V^{\prime}\right)$ are disjoint from $Z$; let $j^{\prime}=k+i+j \in$ $\{1, \ldots, K+J+N-1\}$. Then $f^{k+i+j}(V), \ldots, f^{k+i+j+N-1}(V)$ are disjoint from $Z \cup W$.

- Now consider $V \in \mathcal{V}_{Z}$. As $\mathcal{V}_{Z}$ is a $(N, J)$-wandering cover of $W$, there exists $j \in\{1, \ldots, J\}$ such that $f^{j}(V), \ldots, f^{j+N-1}(V)$ are disjoint from $Z$. If $f^{j}(V), \ldots, f^{k+N-1}(V)$ are disjoint from $W$, then they are also disjoint from $W \cup Z$, which is what we want. Otherwise, there exists $V^{\prime} \in \mathcal{V}_{W}$ and $i \in\{1, \ldots, N-1\}$ such that $f^{i+j}(V) \subset V^{\prime}$. By the previous case, we know there exists $k \in\{1, \ldots, K+J=N-1\}$ such that the iterates $f^{k}\left(V^{\prime}\right), \ldots, f^{k+N-1}\left(V^{\prime}\right)$ are disjoint from $Z \cup W$; hence $j^{\prime}=i+j+k \in\{1, \ldots, K+2 N+2 J-1\}$, and the iterates $f^{j^{\prime}}(V), \ldots, f^{j^{\prime}+N-1}(V)$ are disjoint from $Z \cup W$.

Proof of Lemma 3.10. Let $m_{1}, m_{2} \geq 1$ and $\rho>0$ be given. Let $Z$ be a compact set with the $m_{1}$-wandering property, and let $\mathcal{U}$ be an $\left(m_{1}, J\right)$-wandering cover of $Z$. Let $U_{0}$ be an open set given by Theorem 3.7 with the following properties:

- for each connected component $O$ of $\overline{U_{0}}$ and each $j \in\left\{0, \ldots, m_{1}+J-1\right\}$, the diameter of $f^{j}(O)$ is less than $\rho$;

- for each component $O$ of $\overline{U_{0}}$, if $f^{i}(O) \cap Z \neq \emptyset$, for some $i \in\left\{0, \ldots, m_{1}-1\right\}$, then $f^{i}(O)$ is contained in some element of $\mathcal{U}$;

- the iterates $\bar{U}_{0}, f\left(\bar{U}_{0}\right), \ldots, f^{2 m_{1}+m_{2}+J-1}\left(\bar{U}_{0}\right)$ are pairwise disjoint;

- there is a compact set $D_{0} \subset U_{0}$ such that every nonperiodic point $x \in M$ has an iterate in $D_{0}$.

For each component $O$ of $\bar{U}_{0}$, either the first $m_{1}$ iterates $O, f(O), \ldots, f^{m_{1}-1}(O)$ are disjoint from $Z$, or one of these iterates $f^{i}(O)$ is contained in an element of $\mathcal{U}$. In the latter case, there exists a $j \in\left\{1, \ldots, m_{1}+J-1\right\}$ such that the iterates $f^{j}(O), \ldots, f^{j+m_{1}-1}(O)$ are disjoint from $Z$. In either case, there exists an iterate $f^{j}(O)$ of $O, j \in\left\{0, \ldots, m_{1}+J-1\right\}$ of diameter less than $\rho$, whose first $m_{1}$ iterates are disjoint from $Z$. Selecting one such iterate $f^{j}(O)$ for each component of $\overline{U_{0}}$ and 
taking the union of the $f^{j}\left(U_{0} \cap O\right)$ over all the components $O$, we obtain our desired set $U$. The set $D$ is obtained by intersecting the first $2 m_{1}+m_{2}+J-1$ iterates of $D_{0}$ with the components of $U$ and taking their union. One hence obtain the conclusions 1,2 and 4 .

The sets of the form $f^{j+k}\left(U_{0} \cap O\right)$, where the sets $f^{j}\left(U_{0} \cap O\right)$ define $U$ and $k \in\left\{0, \ldots, m_{1}-1\right\}$, are pairwise disjoint, since $\overline{U_{0}}$ is disjoint from its $2 m_{1}+m_{2}+J-1$ first iterates. This gives conclusion 3 and ends the proof of the lemma.

\subsection{Linearizing the germ of dynamics along a nonperiodic orbit}

In this subsection we present an elementary tool which will be used to construct perturbations of a diffeomorphism $f$ using perturbations of linear cocycles.

Lift of $f$ by the exponential map. Let $M$ be a compact manifold endowed with a Riemannian metric. Denote by $\exp : T M \rightarrow M$ the exponential map associated to this metric, and by $R>0$ the radius of injectivity of the exponential map: for every $x \in M$ the exponential map at $x$ induces a diffeomorphism $\exp _{x}: B_{x}(0, R) \rightarrow$ $B(x, R)$ where $B_{x}(0, R) \subset T_{x} M$ and $B(x, R)$ are the balls of radius $R$ centered at $0_{x} \in T_{x} M$ and at $x$, respectively. It is important for our purposes to notice that the derivative $D_{x} \exp _{x}$ can be identified with the identity map of $T_{x} M$.

Let $f$ be a diffeomorphism and let $C>1$ be a bound for $\|D f\|$ and for $\left\|D f^{-1}\right\|$. For each vector $u \in T_{x} M$ with $\|u\|<R$ and $d\left(f(x), f\left(\exp _{x}(u)\right)\right)<R$, we define

$$
\tilde{f}_{x}(u)=\exp _{f(x)}^{-1}\left(f\left(\exp _{x}(u)\right)\right)
$$

This formula defines a diffeomorphism $\tilde{f}: U \rightarrow V$ where

$$
\begin{gathered}
U=\left\{(x, u) \in T M \mid\|u\|<R \quad \text { and } \quad d\left(f(x), f\left(\exp _{x}(u)\right)\right)<R\right\} \\
V=\left\{(x, u) \in T M \mid\|u\|<R \quad \text { and } \quad d\left(f^{-1}(x), f^{-1}\left(\exp _{x}(u)\right)\right)<R\right\} .
\end{gathered}
$$

The sets $U$ and $V$ are neighborhoods of the zero-section of the tangent bundle, both containing the set of vectors whose norm is bounded by $C^{-1} R$. We denote by $\tilde{f}_{x}: U_{x} \rightarrow V_{f(x)}$ the induced diffeomorphism on $U_{x}=U \cap T_{x} M$.

For every $n \in \mathbb{Z}$ we let $U^{n}$ be the domain of definition of $\tilde{f}^{n}$, we let $V_{n}=\tilde{f}^{n}\left(U_{n}\right)$ and we let $\tilde{f}_{x}^{n}: U_{x, n} \rightarrow V_{f^{n}(x), n}$ be the induced map on the fibers.

Linearizing coordinates of $\tilde{f}$ along an orbit. The map $\tilde{f}^{n}$ defines a germ of the dynamics in a neighborhood of the zero section. The differential $D f$ is also a homeomorphism of $T M$ that induces a continuous family of linear diffeomorphisms on the fibers.

For $n \geq 0$, we let $\psi_{n}=D f^{n} \circ \tilde{f}^{-n}: V_{n} \rightarrow T M$ and $\psi_{x, n}: V_{x, n} \rightarrow T_{x} M$ be the induced maps. We have the following properties:

- For every integer $n$, the family $\left\{\psi_{x, n}\right\}_{x \in M}$ is a compact family of $C^{1}$ diffeomorphisms depending continuously (in the $C^{1}$-topology) on $x$. Indeed, $\left\{\tilde{f}_{x}^{-n}\right\}_{x \in M}$ and $\left\{D f_{x}^{n}\right\}_{x \in M}$ are uniformly continuous families of diffeomorphisms. 
- The derivative of the diffeomorphism $\psi_{x, n}$ at the zero vector $0_{x}$ is the identity map:

$$
D_{0_{x}}\left(\psi_{x, n}\right)=I d_{T_{x} M}
$$

- For every $x \in M$ the family of diffeomorphisms $\left\{\psi_{f^{n}(x), n}\right\}_{n \in \mathbb{N}}$ are linearizing coordinates along the orbit of $x$; more precisely, we have the following commutative diagram:

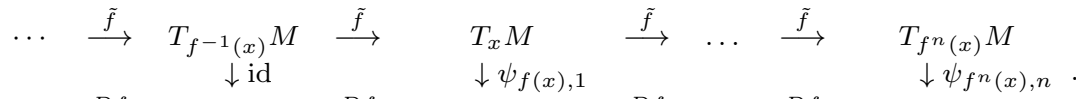

$$
\begin{aligned}
& \quad \ldots \quad \stackrel{D f}{\longrightarrow} T_{f^{-1}(x)} M \quad \stackrel{D f}{\longrightarrow} \quad T_{x} M \quad \stackrel{D f}{\longrightarrow} \quad \ldots \quad \stackrel{D f}{\longrightarrow} \quad T_{f^{n}(x)} M
\end{aligned}
$$

Perturbations of the linearized dynamics. Given a point $x \in M$ we consider the derivative of $f$ along the orbit orb $f(x)$ of $x$ as being a diffeomorphism of the (noncompact, non-connected) manifold $\left.T M\right|_{\mathrm{orb}_{f}(x)}$ endowed with the Euclidean metric on the fibers. Now we will consider perturbations of $D f$ in $\operatorname{Diff}^{1}\left(\left.T M\right|_{\operatorname{orb}_{f}(x)}\right)$ in the corresponding $C^{1}$-topology.

Lemma 3.14. Let $f$ be a diffeomorphism of a compact manifold. For every pair $0<\tilde{\varepsilon}<\varepsilon$ and every $n>0$ there exists $\rho>0$ with the following property.

Consider an open set $U$ whose iterates $f^{i}(U), i \in\{0, \ldots, n\}$, are pairwise disjoint. Fix a point $x \in M$ and assume that $U$ is contained in $B(x, \rho)$; let $\tilde{U}=\exp _{x}^{-1}(U)$. Then we have the following.

1. For every $i \in\{0, \ldots, n\}$, the map $\Psi_{f^{i}(x), i}=\psi_{f^{i}(x), i} \circ \exp _{f^{i}(x)}^{-1}$ induces a diffeomorphism from $f^{i}(U)$ onto $D f^{i}(\tilde{U})$ such that

$$
\max \left\{\left\|D \Psi_{f^{i}(x), i}\right\|,\left\|D \Psi_{f^{i}(x), i}^{-1}\right\|,\left|\operatorname{det} D \Psi_{f^{i}(x), i}\right|,\left|\operatorname{det} D \Psi_{f^{i}(x), i}^{-1}\right|\right\}<2 .
$$

2. For every perturbation $\tilde{g}$ of $D f$ with support in $\bigcup_{i=0}^{n-1} D f^{i}(\tilde{U})$ such that $d_{C^{1}}(\tilde{g}, D f)<\tilde{\varepsilon}$, let $g$ be the map defined by

$$
\begin{aligned}
& \text { - } g(y)=f(y) \text { if } y \notin \bigcup_{i=0}^{n-1} f^{i}(U) \\
& \text { - } g(y)=\Psi_{f^{i+1}(x), i+1}^{-1} \circ \tilde{g} \circ \Psi_{f^{i}(x), i}(y) \text { if } y \in f^{i}(U), i \in\{0, \ldots, n-1\} \text {. }
\end{aligned}
$$

Then the map $g: M \rightarrow M$ is a diffeomorphism. It is a perturbation of $f$ with support in $\bigcup_{i=0}^{n-1} f^{i}(U)$ such that $d_{C^{1}}(g, f)<\varepsilon$.

3. If furthermore $\tilde{g}$ is a tidy perturbation of $D f$, then $g$ is a tidy perturbation of $f$.

Proof. The unique nontrivial statement in this lemma is the fact that $g$ is an $\varepsilon$ perturbation of $f$ if $\tilde{g}$ is an $\tilde{\varepsilon}$ perturbation of $D f$. The proof of this is a simple calculation using the facts that:

- the derivative $D_{0_{x}}\left(\exp _{x}\right)$ is an isometry of $T_{x} M$,

- the derivative $D_{0_{x}} \psi_{x, i}$ is the identity for all $x$ and $i$,

- the family $\left\{\psi_{x, i}, x \in M, 0 \leq i \leq n\right\}$ induces a compact family of $C^{1}$ diffeomorphisms on the balls $\bar{B}\left(0_{x}, \frac{R}{C^{n}}\right)$ having $0_{x}$ as a fixed point. 


\section{4 (LD) property: reduction to a perturbation result in towers}

In this section, we show how to reduce the proof of Theorem B to the following perturbative result. Its proof is deferred to Section 5 .

Theorem B' (Large derivative by perturbation). For any $d \geq 1$ and any $C, K, \varepsilon>$ 0 , there exists an integer $n_{0}=n_{0}(C, K, \varepsilon) \geq 1$ with the following property. Consider any diffeomorphism $f$ of a d-dimensional manifold $M$ with $\|D f\|,\left\|D f^{-1}\right\|<C$ and any $n \geq n_{0}$. Let $N=2^{d+1} n$. Then there exists $\rho_{0}=\rho_{0}(n, f, K, \varepsilon)>0$ such that,

- for any open set $U$ with diameter $<\rho_{0}$ and where the iterates $\bar{U}, f(\bar{U}), \ldots$, $f^{N-1}(\bar{U})$ are pairwise disjoint,

- for any compact set $\Delta \subset U$,

there exists $g \in \operatorname{Diff}^{1}(M)$ such that

- the $C^{1}$ distance from $f$ to $g$ is less than $\varepsilon$;

- $g$ is a tidy perturbation of $f$ with support in $U \cup f(U) \cup \cdots \cup f^{N-1}(U)$;

- for any $x \in \Delta$ there exists $j \in\{0, \ldots, N-n\}$ such that

$$
\max \left\{\left\|D g^{n}\left(g^{j}(x)\right)\right\|,\left\|D g^{-n}\left(g^{j+n}(x)\right)\right\|\right\}>K .
$$

\subsection{Our shopping list for the proof of Theorem B}

Let $M$ be a compact manifold with dimension $d$. Consider a diffeomorphism $f$ whose periodic orbits are hyperbolic, a constant $\varepsilon>0$, a sequence $\left(K_{i}\right)$ that tends to $+\infty$ (for instance $K_{i}=i$ ) and a constant $C>0$ that bounds the norms of $D g$ and $D g^{-1}$, for any diffeomorphism $g$ that is $\varepsilon$-close to $f$ in the $C^{1}$-distance.

Our perturbation $g$ of $f$ will emerge as the limit of a sequence of perturbations $g_{0}=f, g_{1}, g_{2}, \ldots$ Each perturbation $g_{i}$ will satisfy a large derivative property for an interval of times $\left[n_{i}, n_{i+1}-1\right]$. The objects involved at step $i$ in the construction are:

- $g_{i}$, the perturbation, and $\varepsilon_{i}$, the $C^{1}$ distance from $g_{i}$ to $g_{i-1}$.

- $\left[n_{i}, n_{i+1}-1\right]$, the interval of time for which prescribed large derivative for $g_{i}$ occur, and $K_{i}$, the magnitude of large derivative created for these iterates.

- $U_{i}$, an open set such that the iterates $g_{i-1}^{k}\left(\overline{U_{i}}\right), k \in\left\{0, \ldots, m_{i}\right\}$ are pairwise disjoint, where $m_{i}=2^{d+1}\left(n_{i}+n_{1}+1+\cdots+n_{i+1}-1\right)$. The perturbation $g_{i}$ of $g_{i-1}$ is supported in the union of these iterates and is tidy with respect to their union. $\Phi_{i}$ is the $C^{1}$ conjugacy between $g_{i}$ and $f$ such that $\Phi_{i} \circ \Phi_{i-1}^{-1}$ is supported in $\cup_{k=1}^{m_{i}-1} g_{i-1}^{k}\left(U_{i}\right)$. The integer $M_{i}=\prod_{k=1}^{i} C^{m_{k}}$ bounds the $C^{1}$ norm of the conjugacy $\Phi_{i}$.

- $\rho_{i}>0$, an upper bound on the diameter of the first $m_{i}$ iterates of $U_{i}$ under $g_{i-1}$. 
- $D_{i}$, a compact set contained in $U_{i}$ that meets every aperiodic $g_{i-1}$-orbit (and hence also every aperiodic orbit of the tidy perturbation $g_{i}$ ).

- $\Delta_{i}$, a compact set with $D_{i} \subset \operatorname{int} \Delta_{i} \subset \Delta_{i} \subset U_{i}$. Large derivative will occur for $g_{i}$ in the first $m_{i}$ iterates of $\Delta_{i}$.

- $Z_{i}$, a compact set, $x_{i}$, a point, and $N_{i}$, an iterate, such that property (UD) holds for $f$ between $x_{i}$ and points of $Z_{i}$ in time less than $N_{i}$.

Some objects in this construction are fixed prior to the iterative construction of the perturbations, and others are chosen concurrently with the perturbations. Thus we have two types of choices, a priori and inductive, and they are chosen in roughly the following order:

- a priori choices: $\varepsilon_{i}, n_{i}, Z_{i}, x_{i}, N_{i}$;

- inductive choices: $\rho_{i}, U_{i}, D_{i}, \Delta_{i}, g_{i}$.

\subsection{A priori choices}

\subsubsection{Choice of $\varepsilon_{i}, n_{i}, m_{i}, M_{i}$}

We first describe how to select inductively the positive numbers $\varepsilon_{i}$ and the integers $n_{i}$. We initially choose $\varepsilon_{1}<\varepsilon / 2$ and $n_{1}=n_{0}\left(C, 2 K_{1}, \varepsilon_{1}\right)$, according to Theorem B'. Now given $\left(\varepsilon_{i}, n_{i}\right)$, we let

$$
\varepsilon_{i+1}=\min \left\{\varepsilon_{i} / 2, \frac{K_{i-1}}{4 n_{i} C^{n_{i}-1}}\right\} \quad \text { and } \quad n_{i+1}=n_{0}\left(C, 2 K_{i+1}, \varepsilon_{i+1}\right) .
$$

With these definitions we have for any $\ell \geq 1$,

$$
\sum_{k \geq \ell} \varepsilon_{k}<2 \varepsilon_{\ell}<\varepsilon
$$

From this it follows that the sequence of perturbations $\left(g_{i}\right)_{i \in \mathbb{N}}$ such that $d_{C^{1}}\left(g_{i-1}, g_{i}\right)<\varepsilon_{i}$ and $g_{0}=f$ will have a limit $g_{i} \rightarrow g$ as $i \rightarrow \infty$ that is a diffeomorphism satisfying $d_{C^{1}}(f, g)<\varepsilon$. The bound for $\varepsilon_{i}$ is justified by the following lemma which allows us to pass to $g$ the Large Derivative Property satisfied by $g_{i}$.

Lemma 4.1. Suppose that $\varepsilon_{i+1}<\frac{K_{i-1}}{4 n_{i} C^{n_{i}-1}}$. Then, for any $n \leq n_{i}$ and any sequences of matrices $A_{1}, \ldots, A_{n}$ and $B_{1}, \ldots, B_{n}$ satisfying:

- $\left\|A_{k}\right\|,\left\|A_{k}^{-1}\right\|,\left\|B_{k}\right\|,\left\|B_{k}^{-1}\right\|<C$ for $k \in\{1, \ldots, n\}$,

- $\left\|A_{k}-B_{k}\right\|,\left\|A_{k}^{-1}-B_{k}^{-1}\right\|<4 \varepsilon_{i+1}$ for $k \in\{1, \ldots, n\}$,

- $\max \left\{\left\|A_{n} \cdots A_{1}\right\|,\left\|\left(A_{n} \cdots A_{1}\right)^{-1}\right\|\right\}>2 K_{i-1}$,

we have:

$$
\max \left\{\left\|B_{n} \cdots B_{1}\right\|,\left\|\left(B_{n} \cdots B_{1}\right)^{-1}\right\|\right\}>K_{i-1} .
$$


Proof. Without loss of generality we may assume that $\left\|A_{n} \ldots A_{1}\right\|>2 K_{i-1}$. We have:

$$
\begin{aligned}
\left\|A_{n} \cdots A_{1}\right\|-\left\|B_{n} \cdots B_{1}\right\| & \leq \sum_{j=1}^{n}\left\|A_{n} \cdots A_{j+1} \cdot\left(A_{j}-B_{j}\right) \cdot B_{j-1} \cdots B_{1}\right\| \\
& \leq 4 n \varepsilon_{i+1} C^{n-1} \leq 4 n_{i} \varepsilon_{i+1} C^{n_{i}-1} \\
& <K_{i-1}, \text { by our choice of } \varepsilon_{i} .
\end{aligned}
$$

We obtain the desired conclusion: $\left\|B_{n_{i}} \cdots B_{1}\right\|>\left\|A_{n_{i}} \cdots A_{1}\right\|-K_{i-1}>K_{i-1}$.

\subsubsection{Choice of $x_{i}, Z_{i}$ and $N_{i}$}

Assume $f$ has the unbounded distortion property on the wandering set (UD ${ }^{M \backslash \Omega}$ ) and on the stable manifolds $\left(\mathrm{UD}^{s}\right)$. In this section we choose a countable family of pairs $\left(x_{i}, Z_{i}\right)$ where $Z_{i}$ is a compact set disjoint from the orbit of $x_{i}$ which will be used for testing the $\left(\mathrm{UD}^{M \backslash \Omega}\right)$ and the $\left(\mathrm{UD}^{s}\right)$-properties of the perturbations $g_{i}$ we will construct.

By definition of the (UD $\left.\mathrm{UD}^{M \backslash \Omega}\right)$-property there exists a countable and dense subset $\mathcal{X}^{M \backslash \Omega} \subset M \backslash \Omega(f)$ such that, for any $K>0$, any $x \in \mathcal{X}^{M \backslash \Omega}$ and any $y \in M \backslash \Omega(f)$ not in the orbit of $x$, there exists $n \geq 1$ such that:

$$
|\log | \operatorname{det} D f^{n}(x)|-\log | \operatorname{det} D f^{n}(y)||>K .
$$

For every $x \in \mathcal{X}^{M \backslash \Omega}$ we choose a countable set $\mathcal{Z}_{x}$ of compact subsets of $M \backslash \Omega_{f}$ disjoint from the orbit of $x$ and covering the complement in $M \backslash \Omega$ of the orbit of $x$. We define

$$
\mathcal{Z}^{M \backslash \Omega}=\left\{(x, Z) \mid x \in \mathcal{X}^{M \backslash \Omega}, Z \in \mathcal{Z}_{x}\right\} .
$$

By definition of the $\left(\mathrm{UD}^{s}\right)$-property, for any hyperbolic periodic orbit $\mathcal{O}$, there exists a dense countable subset $\mathcal{X}^{\mathcal{O}} \subset W^{s}(\mathcal{O}) \backslash \mathcal{O}$ such that, for any $K>0$, any $x \in \mathcal{X}^{\mathcal{O}}$ and any $y \in W^{s}(\mathcal{O})$ not in the orbit of $x$, there exists $n \geq 1$ such that:

$$
|\log | \operatorname{det} D f_{\mid W^{s}(\mathcal{O})}^{n}(x)|-\log | \operatorname{det} D f_{\mid W^{s}(\mathcal{O})}^{n}(y)||>K .
$$

For every $x \in \mathcal{X}^{\mathcal{O}}$ we choose a countable set $\mathcal{Z}_{x}$ of compact subsets of $W^{s}(\mathcal{O})$ disjoint from the orbit of $x$ and covering the complement in $W^{s}(\mathcal{O})$ of the orbit of $x$. We define

$$
\mathcal{Z}^{\mathcal{O}}=\left\{(x, Z) \mid x \in \mathcal{X}^{\mathcal{O}}, Z \in \mathcal{Z}_{x}\right\}
$$

We set

$$
\mathcal{Z}^{s}=\bigcup_{\mathcal{O} \in \operatorname{Per}(f)} \mathcal{Z}^{\mathcal{O}} \text {, and } \mathcal{Z}=\mathcal{Z}^{M \backslash \Omega} \sqcup \mathcal{Z}^{s} \text { the disjoint union of } \mathcal{Z}^{M \backslash \Omega} \text { and } \mathcal{Z}^{s} \text {. }
$$

Since, by hypothesis, the periodic orbits of $f$ are all hyperbolic, the set of periodic orbits is countable. Consequently $\mathcal{Z}$ is a countable set.

For each pair $(x, Z) \in \mathcal{Z}$, we will need to verify that our perturbations preserve countably many conditions of the form

$$
|\log | \operatorname{det} D g_{i}^{n}(x)|-\log | \operatorname{det} D g_{i}^{n}(y)||>L_{i}, \quad \text { for every } y \in \Phi_{i}(Z),
$$


where $L_{i}$ is a sequence of natural numbers tending to $\infty$. To this end, we fix an enumeration $\left\{\left(x_{i}, Z_{i}\right)\right\}_{i \in \mathbb{N}}$ of the disjoint union $\bigsqcup_{n \in \mathbb{N}} \mathcal{Z}$; each pair $(x, Z) \in \mathcal{Z}$ appears infinitely many times as a pair $\left(x_{i_{k}}, Z_{i_{k}}\right)$ in this choice of indexing.

We now fix a sequence of integers $N_{i}$ as follows:

- If $\left(x_{i}, Z_{i}\right) \in \mathcal{Z}^{M \backslash \Omega}$ then we fix $N_{i}$ such that for every $y \in Z_{i}$ there is $n \in$ $\left\{1, \ldots, N_{i}\right\}$ with

$$
|\log | \operatorname{det} D f^{n}\left(x_{i}\right)|-\log | \operatorname{det} D f^{n}(y)||>K_{i}+4 d \log M_{i} .
$$

- If $\left(x_{i}, Z_{i}\right) \in \mathcal{Z}^{\mathcal{O}}$ for some periodic orbit $\mathcal{O}$ then we fix $N_{i}$ such that for every $y \in Z_{i}$ there exists $n \in\left\{1, \ldots, N_{i}\right\}$ with

$$
|\log | \operatorname{det} D f_{\mid W^{s}(\mathcal{O})}^{n}\left(x_{i}\right)|-\log | \operatorname{det} D f_{\mid W^{s}(\mathcal{O})}^{n}(y)||>K_{i}+4 d \log M_{i} .
$$

The $M_{i}$ appears in these expressions because $i$ will index the $i$ th perturbation in our construction, and the effects of the previous perturbations on the (UD) property will be taken into account. The number $M_{i}^{2}$ bounds the effect of conjugacy by $\Phi_{i}$ on the derivative.

We set $Y_{i, 0}=\bigcup_{k=0}^{N_{i}} f^{k}\left(Z_{i} \cup\left\{x_{i}\right\}\right)$. Lemma 3.12 implies that the sets $Y_{i, 0}$ have the wandering orbit property for $f$.

\subsection{Inductive hypotheses implying Theorem B}

We now describe conditions on the inductively-chosen objects that must satisfied and explain how they imply the conclusion of Theorem B. In a later subsection we explain how these choices can be made so that the inductive conditions are satisfied.

\subsubsection{Conditions on $\rho_{i}$ so that $\left(\Phi_{i}\right)$ converges}

According to Lemma 3.5, the diffeomorphisms $\Phi_{i}$ (conjugating $g_{i}$ to $f$ ) converge uniformly to a homeomorphism $\Phi$ (conjugating $g$ to $f$ ) provided the following conditions hold.

$$
\rho_{i}<2^{-1} \min \left\{\rho_{i-1}, M_{i-1}^{-1}\right\}
$$

\subsubsection{Conditions on $\rho_{i}$ preserving prolonged visits to towers}

Our perturbation at step $i$ will produce prescribed large derivative for $g$ for orbits visiting the compact set $\Delta_{i}$. In order to verify that $g$ has the (LD) property, we will need to know that every nonperiodic orbit for $g$ visits each set $\Delta_{i}$, which imposes the following condition on the sequence $\left(\rho_{i}\right)$ :

$$
\rho_{i+1}<2^{-1} \inf _{k \in\left\{0, \ldots, m_{i}\right\}} d\left(g_{i}^{k}\left(D_{i}\right), M \backslash g_{i}^{k}\left(\Delta_{i}\right)\right) .
$$

The (LD) property on $g$ will be proved in the next subsection by using the following lemma. 
Lemma 4.2. Assume furthermore that condition (3) holds. Then for every $i$ and any $y \in M \backslash \operatorname{Per}(g)$, there exists an integer $t$ such that

$$
g^{t}(y) \in \Delta_{i} \quad \text { and } \quad g_{i}^{t}(x) \in D_{i}
$$

where $x=\Phi_{i} \circ \Phi^{-1}(y)$. For all $k \in\left\{0, \ldots, m_{i}\right\}$, we also have $g^{t+k}(y) \in g_{i}^{k}\left(\Delta_{i}\right)$.

Proof. Fix $j \geq i$. Let $y$ be a non-periodic point for $g_{j}$ and $x=\Phi_{i} \circ \Phi_{j}^{-1}(y)$. For any $n$, we have $g_{j}^{n}(y)=\Phi_{j} \circ \Phi_{i}^{-1} g_{i}^{n}(x)$. The point $x$ is not periodic for $g_{i}$, since $y$ is not periodic for $g_{j}$ and $y$ is the image of $x$ under the conjugacy between $g_{i}$ and $g_{j}$.

By our hypothesis on $D_{i}$, there exists $t$ such that $g_{i}^{t}(x) \in D_{i}$. The hypothesis (3) on $\rho_{i}$ implies that $\Phi_{j} \circ \Phi_{i}^{-1}\left(g_{i}^{k}\left(D_{i}\right)\right) \subset g_{i}^{k}\left(\Delta_{i}\right)$ for $k \in\left\{0, \ldots, 0, \ldots, m_{i}\right\}$, since, by Lemma 3.5, $d_{\text {unif }}\left(\Phi_{j} \circ \Phi_{i}^{-1}, \mathrm{id}\right)<\sum_{k=i+1}^{j} \rho_{k}<2 \rho_{i+1}$. This implies that $g_{j}^{n+k}(y) \in$ $g_{i}^{k}\left(\Delta_{i}\right)$.

Now consider $y \in M$ that is not periodic for $g$. Let $x=\Phi_{i} \circ \Phi^{-1}(y)$. For every $j \geq$ $i$, we set $y_{j}=\Phi_{j} \circ \Phi_{i}^{-1}(x)$; observe that $y_{j} \rightarrow y$ as $j \rightarrow \infty$. The previous argument implies there exists a $t$ such that for every $j$ and every $k \in\left\{0, \ldots, 0, \ldots, m_{i}\right\}$, we have $g_{j}^{t+k}\left(y_{j}\right) \in g_{i}^{k}\left(\Delta_{i}\right)$; compactness of $\Delta_{i}$ implies that $g^{t+k}(y) \in g_{i}^{k}\left(\Delta_{i}\right)$.

\subsubsection{Conditions on $\rho_{i}, U_{i}$ and $g_{i}$ for the (LD) property}

The next lemma imposes one more requirement on $\rho_{i}, U_{i}$ and on the derivative $D g_{i}$ in order to obtain property $(\mathrm{LD})$.

Lemma 4.3 (Final conditions for (LD)). Suppose further that:

- For every $x, y \in M$,

$$
d(x, y)<2 \rho_{i} \Rightarrow\left\|D g_{i-1}(x)-D g_{i-1}(y)\right\|<\varepsilon_{i+1} .
$$

- The support of $g_{i+1}$ is disjoint from the support of $g_{i}$ :

$$
\bar{U}_{i-1}, \ldots, g_{i-1}^{m_{i-1}-1}\left(\bar{U}_{i-1}\right), \bar{U}_{i}, \ldots, g_{i-1}^{m_{i}-1}\left(\bar{U}_{i}\right) \text { are pairwise disjoint. }
$$

- For any $n \in\left[n_{i}, n_{i+1}-1\right]$ and $x \in \Delta_{i}$, there is $j \in\left\{0, \ldots, m_{i}-n\right\}$ such that

$$
\max \left\{\left\|D g_{i}^{n}\left(g_{i}^{j}(x)\right)\right\|,\left\|D g_{i}^{-n}\left(g_{i}^{j+n}(x)\right)\right\|\right\}>2 K_{i} .
$$

Then $g$ has the large derivative (LD) property.

Proof. Let $g$ be the limit of the $g_{i}$. Let $K$ be a large positive number and choose $i_{0}$ such that $K_{i_{0}}>K$. We claim that for every $y \in M \backslash \operatorname{Per}(g)$, and for every $n \geq n_{i_{0}}$, there exists an integer $j>0$ such that

$$
\max \left\{\left\|D g\left(g^{j}(y)\right)\right\|,\left\|D g^{-n}\left(g^{j+n}(y)\right)\right\|\right\}>K .
$$

Fix such an $n \geq n_{i_{0}}$, and choose $i \geq i_{0}$ such that $n \in\left[n_{i}, n_{i+1}-1\right]$. Let $y \in M \backslash \operatorname{Per}(g)$. Let $x=\Phi_{i} \circ \Phi^{-1}(y)$. Note that $x \in M \backslash \operatorname{Per}\left(g_{i}\right)$, so by our hypothesis on $D_{i}$, there exists an integer $t$ such that $g_{i}^{t}(x) \in D_{i}$. Lemma 4.2 implies 
that $g^{t}(y) \in \Delta_{i}$. Since the (LD) property is a property of orbits, it suffices to assume that $y \in \Delta_{i}$ and $x=\Phi_{i} \circ \Phi^{-1}(y) \in D_{i}$.

Now $x \in D_{i} \subset \Delta_{i}$ implies there exists $j \in\left\{0, \ldots, m_{i}-n\right\}$ such that

$$
\max \left\{\left\|D g_{i}^{n}\left(g_{i}^{j}(x)\right)\right\|,\left\|D g_{i}^{-n}\left(g_{i}^{j+n}(x)\right)\right\|\right\}>2 K_{i} .
$$

For any $k \in\left\{0, \ldots, m_{i}-1\right\}$, we have

$$
\begin{aligned}
& \left\|D g\left(g^{k}(y)\right)-D g_{i}\left(g_{i}^{k}(x)\right)\right\| \\
& \quad \leq\left\|D g\left(g^{k}(y)\right)-D g_{i+1}\left(g^{k}(y)\right)\right\|+\left\|D g_{i+1}\left(g^{k}(y)\right)-D g_{i}\left(g_{i}^{k}(x)\right)\right\| \\
& \quad \leq 2 \varepsilon_{i+2}+\left\|D g_{i+1}\left(g^{k}(y)\right)-D g_{i}\left(g^{k}(y)\right)\right\|+\left\|D g_{i}\left(g^{k}(y)\right)-D g_{i}\left(g_{i}^{k}(x)\right)\right\|,
\end{aligned}
$$

where we have used the fact that the $C^{1}$ distance from $g$ to $g_{i+1}$ is bounded by $\sum_{k \geq i+2} \varepsilon_{k}<2 \varepsilon_{i+2}$.

We next bound the remaining terms in the inequality. Since $g^{k}(y)=\Phi \circ$ $\Phi_{i}^{-1}\left(g_{i}^{k}(x)\right)$ and $d_{\text {unif }}\left(\Phi \circ \Phi_{i}^{-1}\right.$, id $)<2 \rho_{i+1}$, the hypothesis (6) implies that

$$
\left\|D g_{i}\left(g^{k}(y)\right)-D g_{i}\left(g_{i}^{k}(x)\right)\right\|<\varepsilon_{i+2} .
$$

By Lemma 4.2, we know that for $k \in\left\{0, \ldots, m_{i}-1\right\}$, the point $g^{k}(y)$ belongs to the set $g_{i}^{k}\left(\Delta_{i}\right)$. Since the support of $g_{i+1}$ is disjoint from $g_{i}^{k}\left(U_{i}\right)$, which contains $g_{i}^{k}\left(\Delta_{i}\right)$, we obtain that $g_{i+1}$ and $g_{i}$ agree in a neighborhood of $g^{k}(y)$, for $k \in\left\{0, \ldots, m_{i}-1\right\}$. From this it follows that $\left\|D g_{i+1}\left(g^{k}(y)\right)-D g_{i}\left(g^{k}(y)\right)\right\|=0$.

We conclude that

$$
\left\|D g\left(g^{k}(y)\right)-D g_{i}\left(g_{i}^{k}(x)\right)\right\| \leq 3 \varepsilon_{i+2} .
$$

But now Lemma 4.1 and the Chain Rule imply that

$$
\max \left\{\left\|D g^{n}\left(g^{j}(y)\right)\right\|,\left\|D g^{-n}\left(g^{j+n}(y)\right)\right\|\right\}>K_{i} .
$$

\subsubsection{The derivative at the periodic orbits is preserved}

Let $\mathcal{O}$ be a periodic orbit of $f$. Note that its image by $\Phi_{i}$ should be disjoint from $\overline{U_{i+1}}, \ldots, g_{i}\left(\overline{U_{i+1}}\right)$ for $i$ large. In particular, the maps $g_{i+1}$ and $g_{i}$ coincide in a neighborhood of the periodic orbit $\Phi_{i}(\mathcal{O})$. This proves that $\Phi(\mathcal{O})=\Phi_{i}(\mathcal{O})$ and that $D g$ coincides with $D g_{i}$ at points of $\Phi(\mathcal{O})$. Since $g_{i}$ and $f$ are conjugate by the diffeomorphism $\Phi_{i}$, we conclude that the derivatives of $f$ on $\mathcal{O}$ and of $g$ on $\Phi(\mathcal{O})$ are conjugate.

\subsubsection{Conditions on $U_{i}$ for preserving the (UD) property}

We assume here that $f$ satisfies the unbounded distortion (UD) property on the stable manifolds and on the wandering set, and we consider the sequences $\left(x_{i}\right),\left(Z_{i}\right)$, $\left(N_{i}\right)$ and $\left(Y_{i, 0}\right)$ defined in Subsection 4.2.2. Let

$$
Y_{i}=\bigcup_{k=0}^{N_{i}} g_{i}^{k}\left(\Phi_{i}\left(Z_{i} \cup\left\{x_{i}\right\}\right)\right)=\Phi_{i}\left(Y_{i, 0}\right) .
$$


We introduce the following condition

$$
\text { The sets } \overline{U_{i}}, \ldots, g_{i-1}^{m_{i}}\left(\overline{U_{i}}\right) \text { are disjoint from } \cup_{k=1}^{i-1} Y_{k} \text {. }
$$

Lemma 4.4. If in addition hypothesis (7) is satisfied, then $g$ has the unbounded distortion properties $(U D)^{M \backslash \Omega}$ and $(U D)^{s}$.

Proof. We prove that $g$ has property (UD) ${ }^{M \backslash \Omega}$; the proof of (UD) $)^{s}$ is similar. Recall the dense set $\mathcal{X}^{M \backslash \Omega}$ in $M \backslash \Omega(f)$ used to define $\left(x_{i}\right)$ and $\left(Z_{i}\right)$. Clearly $\Phi\left(\mathcal{X}^{M \backslash \Omega}\right)$ is dense in $M \backslash \Omega(g)$.

Fix $x \in \Phi\left(\mathcal{X}^{M \backslash \Omega}\right)$ and $y \in M \backslash \Omega(g)$ that are not on the same orbit. Let $K>0$ be some large constant. We claim that there exists $i \in \mathbb{N}$ such that:

- $\Phi^{-1}(x)=x_{i}$,

- $\Phi^{-1}(y) \in Z_{i}\left(\right.$ and we set $\left.y_{i}=\Phi^{-1}(y)\right)$,

- $K_{i}>K$.

Such an $i$ exists because $\Phi^{-1}(y) \in M \backslash\left(\Omega(f) \cup \operatorname{orb}_{f}(x)\right)$ and so, by definition of $\mathcal{Z}_{x}$, there exists $Z \in \mathcal{Z}_{x}$ containing $\Phi^{-1}(y)$. The pair $(x, Z)$ appears as $\left(x_{i}, Z_{i}\right)$ for infinitely many values of $i$. For $i$ sufficiently large, we have $K_{i}>K$, which proves the claim.

By definition of $N_{i}$, there exists $n \in\left\{1, \ldots, N_{i}\right\}$ such that

$$
|\log | \operatorname{det} D f^{n}\left(x_{i}\right)|-\log | \operatorname{det} D f^{n}\left(y_{i}\right)||>K_{i}+4 d \log M_{i} .
$$

Since $g_{i}=\Phi_{i} \circ f \circ \Phi_{i}^{-1}$, and $\left\|D \Phi_{i}\right\|$ and $\left\|D \Phi_{i}^{-1}\right\|$ are both bounded by $M_{i}$, we obtain that

$$
|\log | \operatorname{det} D g_{i}^{n}\left(\Phi_{i}\left(x_{i}\right)\right)|-\log | \operatorname{det} D g_{i}^{n}\left(\Phi_{i}\left(y_{i}\right)\right)||>K_{i} .
$$

Our assumption on the support of the tidy perturbations implies that for every $j>i, g_{j}$ is a tidy perturbation of $g_{j-1}$ whose support is disjoint from the compact set $Y_{i}$. This implies that $g_{j}$ and $g_{i}$ coincide in a neighborhood of the points $\Phi_{i}\left(x_{i}\right), g_{i}\left(\Phi_{i}\left(x_{i}\right)\right), \ldots, g^{N_{i}}\left(\Phi_{i}\left(x_{i}\right)\right)$ and $\Phi_{i}\left(y_{i}\right), g_{i}\left(\Phi_{i}\left(y_{i}\right)\right), \ldots, g^{N_{i}}\left(\Phi_{i}\left(y_{i}\right)\right)$. In particular:

- $\Phi_{j}\left(x_{i}\right)=\Phi_{i}\left(x_{i}\right)$ and $\Phi_{j}\left(y_{i}\right)=\Phi_{i}\left(y_{i}\right)$. Since the points $\Phi_{j}\left(x_{i}\right)$ and $\Phi_{j}\left(y_{i}\right)$ converge to $x$ and $y$ when $j \rightarrow \infty$, it follows that $\Phi_{i}\left(x_{i}\right)=x$ and $\Phi_{i}\left(y_{i}\right)=y$.

- $D g_{i}^{n}\left(\Phi_{i}\left(x_{i}\right)\right)=D g_{j}^{n}(x)$ and $D g_{i}^{n}\left(\Phi_{i}\left(y_{i}\right)\right)=D g_{j}^{n}(y)$.

It follows that, for $j \geq i$,

$$
|\log | \operatorname{det} D g_{j}^{n}(x)|-\log | \operatorname{det} D g_{j}^{n}(y)|| \geq K_{i},
$$

Since $D g_{j}^{n}$ tends to $D g^{n}$ as $j \rightarrow \infty$, it follows that

$$
|\log | \operatorname{det} D g^{n}(x)|-\log | \operatorname{det} D g^{n}(y)|| \geq K_{i}>K,
$$

which concludes the proof of the $\left(\mathrm{UD}^{M \backslash \Omega}\right)$ property for $g$. The proof of the $\left(\mathrm{UD}^{s}\right)$ property is completely analogous. 


\subsection{Satisfying the inductive hypotheses}

To finish the proof of Theorem B (assuming Theorem B'), we are left to explain how to construct inductively $\rho_{i}, U_{i}, D_{i}, \Delta_{i}$ and $g_{i}$ satisfying the properties stated at Section 4.1 and properties (2), (3), (44), (5), (6) and (7). For the construction we require the following extra property:

The sets $g_{i-1}^{j}\left(\overline{U_{i}}\right)$ for $j \in\left\{0, \ldots, m_{i}+m_{i+1}-1\right\}$ are pairwise disjoint.

In the following we assume that all the objects have been constructed up to $\rho_{i}, U_{i}, D_{i}, \Delta_{i}, g_{i}$, and we will construct $\rho_{i+1}, U_{i+1}, D_{i+1}, \Delta_{i+1}, g_{i+1}$.

The constant $\rho_{i+1}$. We choose $\rho_{i+1}$ satisfying:

1. $\rho_{i+1}$ is strictly less than the numbers $\rho_{0}\left(n, g_{i}, 2 K_{i+1}, \varepsilon_{i+1}\right)$ given by Theorem B' for $n_{i+1} \leq n<n_{i+2}$;

2. $\rho_{i+1}<2^{-1} \min \left\{\rho_{i}, M_{i}^{-1}\right\}$,

3. $\rho_{i+1}<2^{-1} \inf _{k \in\left\{0, \ldots, m_{i}\right\}} d\left(g_{i}^{k}\left(D_{i}\right), M \backslash g_{i}^{k}\left(\Delta_{i}\right)\right)$.

4. $\rho_{i+1}$ is less than the Lebesgue number associated to $D g_{i}$ for $\varepsilon_{i+2}$, so that, for every $x, y \in M$, if $d(x, y)<2 \rho_{i+1}$, then $\left\|D g_{i}(x)-D g_{i}(y)\right\|<\varepsilon_{i+2}$.

In particular conditions (2), (3) and (4) are satisfied by $\rho_{i+1}$.

The sets $U_{i+1}, D_{i+1}, \Delta_{i+1}$. Observe that $Y_{k}=\Phi_{k}\left(Y_{k, 0}\right)=\Phi_{i}\left(Y_{k, 0}\right)$ for $k \leq i$ have the wandering orbit property for $g_{i}$ because $Y_{k, 0}$ have the wandering orbit property for $f$. By induction property (8) is satisfied by $U_{i}$ and implies that the sets $g_{i}^{j}\left(\overline{U_{i}}\right)$ for $j \in\left\{0, \ldots, m_{i}+m_{i+1}-1\right\}$ are pairwise disjoint. By Lemma 3.12, the set $\bigcup_{j=0}^{m_{i}-1} g_{i}^{j}\left(\bar{U}_{i}\right)$ has the $m_{i+1}$-wandering orbit property and so Lemma 3.13 implies that $\bigcup_{k=0}^{i} Y_{k} \cup \bigcup_{j=0}^{m_{i}-1} g_{i}^{j}\left(\bar{U}_{i}\right)$ has the $m_{i+1}$-wandering orbit property. Lemma 3.10 gives an open set $U_{i+1}$ and a compact set $D_{i+1} \subset U_{i+1}$ such that

1. the diameter of each connected component of $g_{i}^{j}\left(\overline{U_{i+1}}\right), j \in\left\{0, \ldots, m_{i+1}\right\}$ is less than $\rho_{i+1}$;

2. the sets $g_{i}^{j}\left(\overline{U_{i+1}}\right), j \in\left\{0, \ldots, m_{i+1}-1\right\}$, are disjoint from the sets $Y_{k}, k \leq i$, and from the sets $g_{i}^{j}\left(\bar{U}_{i}\right), j \in\left\{0, \ldots, m_{i+1}-1\right\}$;

3. the sets $g_{i}^{j}\left(\overline{U_{i+1}}\right)$ for $j \in\left\{0, \ldots, m_{i+1}+m_{i+2}-1\right\}$ are pairwise disjoint;

4. every nonperiodic point $x \in M$ has an iterate in $D_{i+1}$.

In particular, conditions (5), (17) and (8) are satisfied by $U_{i+1}$. We next fix some compact set $\Delta_{i+1} \subset U_{i+1}$ containing $D_{i+1}$ in its interior. 
The perturbation $g_{i+1}$. For each $n \in\left\{n_{i+1}, \ldots, n_{i+2}-1\right\}$, we will make a tidy perturbation $g_{n, i+1}$ producing the large derivative at the $n$th iterate. Furthermore, these perturbations will have pairwise disjoint support.

To do this, we partition $\left\{0,1, \ldots, m_{i+1}-1\right\}$ into intervals of the form $I_{n}=$ $\left\{\alpha_{n}, \ldots, \alpha_{n+1}-1\right\}, n_{i+1} \leq n<n_{i+2}$, where $\alpha_{n}=2^{d+1}\left(n_{i+1}+\cdots+n-1\right)$. This is possible since $m_{i+1}=2^{d+1}\left(n_{i+1}+\left(n_{i+1}+1\right)+\cdots+n_{i+2}-1\right)$.

The sets $g_{i}^{j}\left(\overline{U_{i+1}}\right)$ for $j \in I_{n}$ are pairwise disjoint and have a diameter less than $\rho_{0}\left(n, g_{i}, 2 K_{i+1}, \varepsilon_{i+1}\right)$. Hence, we can apply Theorem B' to obtain a tidy perturbation $g_{n, i+1}$ of $g_{i}$ with support in $\bigcup_{j \in I_{n}} g_{i}^{j}\left(U_{i+1}\right)$ such that $d_{C^{1}}\left(g_{n, i+1}, g_{i}\right)<\varepsilon_{i+1}$ and for any $x \in g_{i}^{\alpha_{n}}\left(\Delta_{i+1}\right)$, there exists $j \in\left\{\alpha_{n}, \ldots, \alpha_{n+1}-n\right\}$ such that

$$
\max \left\{\left\|D g_{n, i+1}^{n}\left(g_{n, i+1}^{j}(x)\right)\right\|,\left\|D g_{n, i+1}^{-n}\left(g_{n, i+1}^{j+n}(x)\right)\right\|\right\}>2 K_{i+1} .
$$

We denote by $\varphi_{n, i+1}$ the conjugating diffeomorphism associated to the tidy perturbation $g_{n, i+1}$, which is the identity outside of $\bigcup_{j \in I_{n}} g_{i}^{j}\left(U_{i+1}\right)$. Notice that $\varphi_{n, i+1}$ is also the identity map on $g_{i}^{\alpha_{n}}\left(U_{i+1}\right)$.

We now define the diffeomorphisms $g_{i+1}$ and $\varphi_{i+1}$ that coincide respectively with $g_{i}$ and $\operatorname{id}_{M}$ on $M \backslash \bigcup_{j=0}^{m_{i+1}-1} g_{i}^{j}\left(U_{i+1}\right)$ and with $g_{n, i+1}$ and $\varphi_{n, i+1}$ on $\bigcup_{j \in I_{n}} g_{i}^{j}\left(U_{i+1}\right)$, for $n \in\left\{n_{i}, \ldots, n_{i+1}-1\right\}$. Using the fact that the tidy perturbations $g_{n, i+1}$ have disjoint support, we obtain that $g_{i+1}$ and $\varphi_{i+1}$ have the following properties:

- $g_{i+1}$ is a tidy perturbation of $g_{i}$ with support in $\bigcup_{j=0}^{m_{i+1}-1} g_{i}^{j}\left(U_{i+1}\right)$.

- $g_{i+1}$ is conjugate to $g_{i}$ by $\varphi_{i+1}$.

- $\varphi_{i+1}$ is the identity map on each $g_{i}^{\alpha_{n}}\left(U_{i+1}\right)$; in particular, if $x \in \Delta_{i+1}$ then $g_{i+1}^{\alpha_{n}}(x) \in g_{i}^{\alpha_{n}}\left(\Delta_{i+1}\right)$.

- Consequently, for any $n \in\left\{n_{i+1}, \ldots, n_{i+2}-1\right\}$ and any $x \in \Delta_{i+1}$, there exists $j \in\left\{\alpha_{n}, \alpha_{n+1}-n\right\} \subset\left\{0, \ldots, m_{i+1}-n\right\}$ such that

$$
\max \left\{\left\|D g_{i+1}^{n}\left(g_{i+1}^{j}(x)\right)\right\|,\left\|D g_{i+1}^{-n}\left(g_{i+1}^{j+n}(x)\right)\right\|\right\}>2 K_{i+1} .
$$

The proof of Theorem B assuming Theorem B' is now complete.

\section{Large derivative by perturbation in towers}

The aim of this section is to prove Theorem B', thereby completing the proof of Theorem B. In the first three subsections we reduce the problem to a linear algebra result, which is proved in the last section.

\subsection{Reduction to cocycles}

To any sequence $\left(A_{i}\right)$ in $G L(d, \mathbb{R})$ we associate its linear cocycle as the map $f: \mathbb{Z} \times$ $\mathbb{R}^{d} \rightarrow \mathbb{Z} \times \mathbb{R}^{d}$ defined by $(i, v) \mapsto\left(i+1, A_{i}(v)\right)$. Theorem $\mathrm{B}^{\prime}$ is a consequence of the following corresponding result for $C^{1}$ perturbations of linear cocycles. 
Proposition 5.1. For any $d \geq 1$ and any $C, K, \varepsilon>0$, there exists $n_{1}=$ $n_{1}(d, C, K, \varepsilon) \geq 1$ with the following property.

Consider any sequence $\left(A_{i}\right)$ in $G L(d, \mathbb{R})$ with $\left\|A_{i}\right\|,\left\|A_{i}^{-1}\right\|<C$ and the associated linear cocycle $f$. Consider any integer $n \geq n_{1}$ and let $N=2^{d+1} n$. Then for any open set $U \subset[-1,1]^{d}$ and for any compact set $\Delta \subset U$, there exists a diffeomorphism $g$ of $\mathbb{Z} \times \mathbb{R}^{d}$ such that:

1. the $C^{1}$-distance from $g$ to $f$ is bounded by $\varepsilon$;

2. $g$ is a tidy perturbation of $f$ supported on $\bigcup_{i=0}^{N-1} f^{i}(\{0\} \times U)$;

3. for any $x \in\{0\} \times \Delta$ there exists $j \in\{0, \ldots, N-n\}$ such that

$$
\max \left\{\left\|D g^{n}\left(g^{j}(x)\right)\right\|,\left\|D g^{-n}\left(g^{j+n}(x)\right)\right\|\right\}>K \text {. }
$$

Proof of Theorem B' from Proposition 5.1. Fix $\tilde{\varepsilon}<\varepsilon$, let $\tilde{K}=4 K$ and set $n_{0}=n_{1}(d, C, \tilde{K}, \tilde{\varepsilon})$, according to Proposition 5.1. Let $f$ be a diffeomorphism of the $d$-manifold $M$ with $\|D f\|,\left\|D f^{-1}\right\|<C$ and fix $n \geq n_{0}$. Let $N=2^{d+1} n$. We choose $\rho_{0}=\rho$ according to Lemma 3.14 associated to $\tilde{\varepsilon}<\varepsilon$ and $N$.

Let us show that $\rho_{0}$ satisfies the conclusions of Theorem B'. Let $U \subset M$ be an open set with diameter less than $\rho_{0}$ whose iterates $U, f(U), \ldots, f^{N-1}(U)$ are pairwise disjoint, and let $\Delta \subset U$ be compact. Fix a point $x_{0} \in U$.

Lemma 3.14 asserts that there are diffeomorphisms $\Psi_{f^{i}\left(x_{0}\right), i}: f^{i}(U) \rightarrow T_{f^{i}\left(x_{0}\right)} M$, $i \in\{0, \ldots, N-1\}$ which conjugate $f$ to its tangent map: if $y \in f^{i}(U), i \in\{0, \ldots, N-$ $1\}$ then

Moreover,

$$
f(y)=\Psi_{f^{i+1}\left(x_{0}\right), i+1}^{-1} \circ D_{f^{i}\left(x_{0}\right)} f \circ \Psi_{f^{i}\left(x_{0}\right), i}(y) .
$$

- the quantities $\left\|D \Psi_{f^{i}\left(x_{0}\right), i}\right\|$ and $\left\|D \Psi_{f^{i}\left(x_{0}\right), i}^{-1}\right\|$ are bounded by 2 ;

- any tidy $\tilde{\varepsilon}$-perturbation $\tilde{g}$ of $D f$ with support in $\bigcup_{0}^{N-1} D f^{i}(\tilde{U})$, where $\tilde{U}=$ $\Psi_{x_{0}, 0}(U)$, induces a tidy $\varepsilon$-perturbation $g$ of $f$ supported on $\bigcup_{0}^{N-1} f^{i}(U)$, through a conjugacy by the diffeomorphisms $\Psi_{f^{i}\left(x_{0}\right), i}$.

We apply Proposition 5.1 to the cocycle induced by $D f$ on the tangent bundle $\left.T M\right|_{\operatorname{orb}\left(x_{0}\right)}$ over the orbit of $x_{0}$, to the images $\tilde{U}, \tilde{\Delta}$ of $U, \Delta$ by $\Psi$, and to the integer $n$ (which is larger than $n_{1}$ ). We obtain a (nonlinear) cocycle $\tilde{g}: \mathbb{Z} \times \mathbb{R}^{d} \rightarrow \mathbb{Z} \times \mathbb{R}^{d}$ whose $C^{1}$-distance to $D f$ is smaller than $\varepsilon$, which is a tidy perturbation of $D f$ supported in $\bigcup_{i=0}^{N-1} D f^{i}(\tilde{U})$ and such that for any $x \in \tilde{\Delta}$ there exists $j \in\{0, \ldots, N-n\}$ satisfying

$$
\max \left\{\left\|D \tilde{g}^{n}\left(\tilde{g}^{j}(x)\right)\right\|,\left\|D \tilde{g}^{-n}\left(\tilde{g}^{j+n}(x)\right)\right\|\right\}>\tilde{K} .
$$

By Lemma 3.14, the cocycle $\tilde{g}$ defines a tidy perturbation $g$ of $f$ supported on $\bigcup_{0}^{N-1} f^{i}(U)$ and such that $d_{C^{1}}(f, g)<\varepsilon$. Consider $x \in \Delta, \tilde{x}=\Psi_{x_{0}, 0}(x) \in \tilde{\Delta}$ and the integer $j \in\{0, \ldots, N-n\}$ such that (9) holds. Note that on $g^{j}(U)$ we have $\tilde{g}^{n}=\Psi_{f^{j+n}\left(x_{0}\right), j+n} \circ \tilde{g}^{n} \circ \Psi_{f^{j}\left(x_{0}\right), j}^{-1}$. Since the derivatives $\left\|\Psi_{f^{j+n}\left(x_{0}\right), j+n}\right\|$ and $\left\|\Psi_{f^{j}\left(x_{0}\right), j}^{-1}\right\|$ are bounded by 2 , it follows that

$$
\left\|D g^{n}\left(g^{j}(x)\right)\right\| \geq \frac{1}{4}\left\|D \tilde{g}^{n}\left(\tilde{g}^{j}(\tilde{x})\right)\right\|,
$$




$$
\left\|D g^{-n}\left(g^{j+n}(x)\right)\right\| \geq \frac{1}{4}\left\|D \tilde{g}^{-n}\left(\tilde{g}^{j+n}(\tilde{x})\right)\right\| .
$$

By property (91) above, this gives

$$
\max \left\{\left\|D g^{n}\left(g^{j}(x)\right)\right\|,\left\|D g^{-n}\left(g^{j+n}(x)\right)\right\|\right\}>\frac{1}{4} \tilde{K}=K .
$$

\subsection{Reduction to a perturbation result in a cube}

We now reduce Proposition 5.1 to the case $U$ is an iterate of the interior of the standard cube $Q=[-1,1]^{d}$ and $\Delta$ is an iterate of a smaller closed cube $\delta Q$.

Proposition 5.2. For any $d \geq 1, C, K, \varepsilon>0$ and $\delta \in(0,1)$, there exists an integer $n_{2}=n_{2}(d, C, K, \varepsilon, \delta) \geq 1$ with the following property.

Consider any sequence $\left(A_{i}\right)$ in $G L(d, \mathbb{R})$ with $\left\|A_{i}\right\|,\left\|A_{i}^{-1}\right\|<C$ and the associated linear cocycle $f$. Then, for any $n \geq n_{2}$ there exists a diffeomorphism $g$ of $\mathbb{Z} \times \mathbb{R}^{d}$ such that:

1. the $C^{1}$-distance from $g$ to $f$ is bounded by $\varepsilon$;

2. $g$ is a tidy perturbation of $f$ supported on $\bigcup_{i=0}^{n-1} f^{i}(\{0\} \times Q)$;

3. there exists $k \in\{0, \ldots, n\}$ such that for any $x \in\{0\} \times \delta Q$ :

$$
\max \left\{\left\|D g^{n}\left(g^{-k}(x)\right)\right\|,\left\|D g^{-n}\left(g^{n-k}(x)\right)\right\|\right\}>K .
$$

Remark 5.3. In Proposition 5.2, the time interval of the perturbation is $\{0, \ldots, n-$ $1\}$, and its support is $\bigcup_{i=0}^{n-1} f^{i}(\{0\} \times Q)$. The time $-k \in\{-n, \ldots, 0\}$ where we see the large derivative property might actually lie outside of the time interval of the perturbation, and so we cannot compose two such perturbations with disjoint support without potentially destroying the large derivative property. Hence we need to consider the effective support of the perturbation, which is the larger set $\bigcup_{i=-n}^{n-1} f^{i}(\{0\} \times Q)$ corresponding to the time interval $\{-n, \ldots, n-1\}$. Note that the unique value of $i \in\{-n, \ldots, n-1\}$ for which we know that that the intersection of the effective support of the perturbation with $\{i\} \times \mathbb{R}^{d}$ is a cube is $i=0$.

Proof of Proposition 5.1, assuming Proposition 5.2, We first define the integer $n_{1}$. Let $C, K, \varepsilon>0$ be given. Define $K_{0}=K^{2^{d+2}}, C_{1}=C . K_{0}^{2}, \varepsilon_{2}=\frac{\varepsilon}{K_{0}^{2}}$, and $K_{1}=K_{0}^{3}$. We fix $\delta=\frac{9}{10}$ and set:

$$
n_{1}(d, C, K, \varepsilon)=n_{2}\left(d, C_{1}, K_{1}, \varepsilon_{2}, \delta\right) .
$$

Now consider $\left(A_{i}\right), N, \Delta$ and $U$ as in the statement of Proposition [5.1. Note that we may assume that for any $j \in\{0, \ldots, N-n\}$, the product $A_{n-1+j} \cdots A_{j}$ and its inverse have a norm bounded by $K$ : otherwise, the conclusion of Proposition 5.1 is satisfied for the trivial perturbation $g=f$ and this value of $k$.

In order to apply Proposition 5.2 we will tile the support of the perturbation. Recall our Remark 5.3 that for a perturbation supported at times $0, \ldots, 2 n-1$, the 
tiles we use will have to be cubes "at time $n$ " and not "at time 0 ". Thus we will tile the images $\{n\} \times \tilde{U}$ and $\{n\} \times \tilde{\Delta}$ of $\{0\} \times U$ and $\{0\} \times \Delta$ under $f^{n}$, where

$$
\tilde{U}=A_{n-1} \ldots A_{0}(U) \text { and } \tilde{\Delta}=A_{n-1} \ldots A_{0}(\Delta) .
$$

Let $a>0$ be such that $\frac{2 a}{\delta} \sqrt{d}$ is less than the distance between $\tilde{\Delta}$ and the complement of $\tilde{U}$. We consider a regular tiling of $\mathbb{R}^{d}$ by cubes

$$
\tilde{Q}_{i_{1}, \ldots, i_{d}}=[-a, a]^{d}+\left(2 i_{1} a, \ldots, 2 i_{d} a\right), \text { where } i_{j} \in \mathbb{Z} .
$$

We also consider the enlarged cubes

$$
Q_{i_{1}, \ldots, i_{d}}=\left[-\frac{a}{\delta}, \frac{a}{\delta}\right]^{d}+\left(2 i_{1} a, \ldots, 2 i_{d} a\right) .
$$

The cubes $Q_{i_{1}, \ldots, i_{d}}$ have diameter equal to $\frac{2 a}{\delta} \sqrt{d}$. By our choice of $a$, any cube $Q_{i_{1}, \ldots, i_{d}}$ such that $\tilde{Q}_{i_{1}, \ldots, i_{d}}$ intersects the compact set $\Delta$ is entirely contained in $U$. We denote by $\Gamma$ the family of the cubes $Q_{i_{1}, \ldots, i_{d}}$ such that $\tilde{Q}_{i_{1}, \ldots, i_{d}} \cap \Delta \neq \emptyset$. Observe that two cubes $Q_{i_{1}, \ldots, i_{d}}$ and $Q_{j_{1}, \ldots, j_{d}}$ are disjoint if and only if there exists $\ell \in\{1, \ldots, d\}$ with $\left|i_{\ell}-j_{\ell}\right| \geq 2$.

Now consider the families $\left\{\Gamma_{\mu}\right\}_{\mu \in\{0,1\}^{d}}$, where $\Gamma_{\mu_{1}, \ldots, \mu_{d}}$ is the collection of cubes $Q_{i_{1}, \ldots, i_{d}} \in \Gamma$ such that the index $i_{\ell}$ is even if $\mu_{\ell}=0$ and odd if $\mu_{\ell}=1$. This gives $2^{d}$ families of pairwise disjoint cubes contained in $\tilde{U}$ such that the union of the corresponding smaller cubes $\tilde{Q}_{i_{1}, \ldots, i_{d}}$ covers $\tilde{\Delta}$. To each $\left(\mu_{1}, \ldots, \mu_{d}\right) \in\{0,1\}^{d}$ we associate the integer $\ell=\sum_{i=1}^{d} \mu_{i} 2^{i-1}$ (this formula induces a bijection from $\{0,1\}^{d}$ to $\left.\left\{0, \ldots, 2^{d}-1\right\}\right)$, and for simplicity we will use the notation $\Gamma_{\ell}$ to denote the family $\Gamma_{\mu_{1}, \ldots, \mu_{d}}$. We can thus write our partition $\Gamma=\Gamma_{0} \cup \cdots \cup \Gamma_{2^{d}-1}$.

We will construct a tidy perturbation $g_{Q}$ of $f$ for each cube $Q \in \Gamma$ such that the supports of the perturbations $g_{Q}$ are pairwise disjoint. The ultimate perturbation $g$ will be obtained by combining all of these perturbations, setting $g$ to equal $g_{Q}$ on the support of $g_{Q}$ and to equal $f$ outside the union of the supports. For each cube $Q \in \Gamma_{\ell}$, for $\ell \in\left\{0, \ldots, 2^{d}-1\right\}$, the time interval of the perturbation will be $I_{\ell}=\{2 n \ell, \ldots, 2 n(\ell+1)-1\} \subset\{0, \ldots, N-1\}$ and the support of $g_{Q}$ will be

$$
W_{Q}=\bigcup_{i \in I_{\ell}} f^{i}(\{n\} \times Q)
$$

The time intervals $I_{\ell}$ form a partition of $\{0, \ldots, N-1\}$ into $2^{d}$ intervals of length $2 n$, which implies that $W_{Q} \cap W_{Q^{\prime}}=\emptyset$ for $Q \in \Gamma_{\ell}, Q^{\prime} \in \Gamma_{\ell^{\prime}}$ with $\ell \neq \ell^{\prime}$. If $Q, Q^{\prime} \in \Gamma_{\ell}$, then the supports are disjoint as well, because $Q$ and $Q^{\prime}$ are disjoint.

We will now use Proposition 5.2 to construct the perturbation $g_{Q}$ on $W_{Q}$. However there is an issue (as explained in Remark 5.3): the time interval $I_{\ell}$ has length $2 n$, as required, but the set $f^{2 n(\ell-1)}(\{n\} \times Q)$ is not (in general) a cube (unless $\ell=0)$, and so does not satisfy the hypotheses of the proposition. For this reason, for $Q \in \Gamma_{\ell}$ and the corresponding smaller cube $\tilde{Q} \subset Q$ we first consider

$$
\widehat{W_{Q}}=f^{-2 n \ell}\left(W_{Q}\right)=\bigcup_{j=0}^{2 n-1} f^{j}(\{n\} \times Q) .
$$


Proposition 5.2 ensures the existence of a tidy $\varepsilon_{2}$-perturbation $\widehat{g_{Q}}$ of $f$, supported in $\bigcup_{i=n}^{2 n-1} f^{i}(\{n\} \times Q) \subset \widehat{W_{Q}}$ and of $k \in\{0, \ldots, n\}$ such that for every $x \in\{n\} \times \tilde{Q}$ :

$$
\max \left\{\left\|D{\widehat{g_{Q}}}^{n}\left(f^{-k}(x)\right)\right\|,\left\|D{\widehat{g_{Q}}}^{-n}\left({\widehat{g_{Q}}}^{n} \circ f^{n-k}(x)\right)\right\|\right\}>K_{1} \text {. }
$$

We now define

$$
g_{Q}=f^{2 n \ell} \circ \widehat{g_{Q}} \circ f^{-2 n \ell}
$$

Observe that:

- $g_{Q}$ is a tidy perturbation of $f$ supported in $\bigcup_{i=2 n \ell+n}^{2 n(\ell+1)-1} f^{i}(\{n\} \times Q) \subset W_{Q}$,

- using the bound on the products $A_{j+n-1} \ldots A_{j}$ and the choice of $\varepsilon_{2}$, we have:

$$
d_{C^{1}}\left(g_{Q}, f\right) \leq\left(\max \left\{\left\|D f^{n}\right\|,\left\|D f^{-n}\right\|\right\}\right)^{4 \ell} d_{C^{1}}\left(\widehat{g_{Q}}, f\right) \leq K^{2^{d+2}} \varepsilon_{2} \leq \varepsilon,
$$

- for every $x \in\{n\} \times \tilde{Q}$ :

$$
\max \left\{\left\|D g_{Q}{ }^{n}\left(f^{2 n \ell-k}(x)\right)\right\|,\left\|D g_{Q}{ }^{-n}\left(g_{Q}{ }^{n} \circ f^{2 n \ell-k}(x)\right)\right\|\right\}>\frac{K_{1}}{K^{2^{d+2}}}>K .
$$

We finally define $g$ by $g=g_{Q}$ on $W_{Q}$ for $Q \in \Gamma$ and $g=f$ outside the union of the $W_{Q}, Q \in \Gamma$. Since the support of the tidy $\varepsilon$-perturbations $g_{Q}$ are disjoint we get that $g$ is a tidy $\varepsilon$-perturbation of $f$ supported on $\bigcup_{i=0}^{N-1} f^{i}(\{0\} \times U)$, proving the two first conclusions of the proposition. Furthermore, since these perturbations are tidy, for every point $x \in\{0\} \times \mathbb{R}^{d}$ and every $\ell \in \mathbb{Z}$ we have $f^{2 n \ell}(x)=g^{2 n \ell}(x)$. What is more, since the support of the perturbation $g_{Q}$ is confined to the final $n$ iterates in the time interval $I_{n}$, we have that $f^{2 n \ell+n-k}(x)=g^{2 n \ell+n-k}(x)$, for any $j \in\{-n, \ldots, 0\}$.

Consider now a point $x \in\{0\} \times \Delta$. We show that there exists a $j$ such that

$$
\max \left\{\left\|D g^{n}\left(g^{j}(0, w)\right)\right\|,\left\|D g^{-n}\left(g^{n+j}(0, w)\right)\right\|\right\}>K \text {. }
$$

By our choice of cubes $Q$ covering $\tilde{\Delta}$, there exists a smaller cube $\tilde{Q}$ such that $f^{n}(x) \in\{n\} \times \tilde{Q}$. The corresponding cube $Q$ belongs to some $\Gamma_{\ell}$. The argument above shows that there exists $k \in\{0, \ldots,-n\}$ such that (10) holds for $f^{n}(x)$. Using the fact that $f^{2 n \ell+n-k}(x)=g^{2 n \ell+n-k}(x)$, for any $k \in\{0, \ldots, n\}$, we obtain that:

$$
\max \left\{\left\|D g^{n}\left(g^{j}(x)\right)\right\|,\left\|D g^{-n}\left(g^{n+j}(x)\right)\right\|\right\}>K,
$$

where $j=2 n \ell+n-k$. This gives the last conclusion of the proposition.

\subsection{Reduction to linear perturbations}

We first define notation and review some linear algebra. For any element $A \in$ $G L(d, \mathbb{R})$ we define the eccentricity of $A$, denoted by $e(A)$, to be the ratio

$$
\sup \left\{\frac{\|A(u)\|}{\|A(v)\|} \text { for } u, v \in \mathbb{R}^{d},\|u\|=\|v\|=1\right\}=\|A\| \cdot\left\|A^{-1}\right\| .
$$


Note that $e(A) \geq 1, e\left(A^{-1}\right)=e(A)$, and for any $B \in G L(d, \mathbb{R})$, we have $\left\|A B A^{-1}\right\| \leq$ $e(A)\|B\|$. We will also use the conorm notation $\mathcal{M}(A)=\left\|A^{-1}\right\|^{-1}$. We recall the basic fact from linear algebra that for any $A \in G L(d, \mathbb{R})$, there exist orthogonal unit vectors $u$ and $v$ such that $\|A u\|=\|A\|$ and $\|A v\|=\mathcal{M}(A)$.

Proposition 5.2 is a consequence of the following proposition.

Proposition 5.4. For any $d \geq 1$ and $C, K, \eta>0$, there exists $n_{3}=n_{3}(d, C, K, \eta) \geq$ 1 with the following property.

For any sequence $\left(A_{i}\right)$ in $G L(d, \mathbb{R})$ satisfying $\left\|A_{i}\right\|,\left\|A_{i}^{-1}\right\|<C$ and $n \geq n_{3}$, there exist $H_{0}, \ldots, H_{r-1}, 0 \leq r \leq \frac{n}{2}$, in $G L(d, \mathbb{R})$ and $k \in\{0, \ldots, n\}$ such that:

- The product $P=A_{-k+n-1} \cdots A_{0} H_{r-1} \cdots H_{0} A_{-1} \cdots A_{-k}$ satisfies the estimate $\max \left\{\|P\|,\left\|P^{-1}\right\|\right\}>K$.

- For $0 \leq i<r$, the map $H_{i}$ sends the standard cube $Q$ into itself.

- For $0 \leq i \leq r$, there is the control

$$
\left(e\left(A_{n-i-1} \cdots A_{0}\right)+e\left(A_{i-1} \cdots A_{0}\right)\right) \cdot\left\|H_{i}-\mathrm{id}\right\|<\eta .
$$

We also state without proof a standard $C^{1}$ perturbation lemma.

Lemma 5.5. For every $d \geq 1, \delta \in(0,1)$ there exists a neighborhood $\mathcal{O}$ of id in $G L(d, \mathbb{R})$ and for any $H \in \mathcal{O}$ there exists a diffeomorphism $h(H)$ of $\mathbb{R}^{d}$ such that

- $h(H)$ coincides with the identity map on $\mathbb{R}^{d} \backslash Q$ and with $H$ on $\delta Q$.

- The map $H \mapsto h(H)$ is $C^{1}$ in the $C^{1}$-topology.

Proof of Proposition 5.2 from Proposition 5.4. Lemma 5.5 associates to any $H$ in a neighborhood $\mathcal{O}$ of id in $G L(d, \mathbb{R})$ a diffeomorphism $h(H)$ that satisfies

$$
d_{C^{1}}(h(H), \mathrm{id}) \leq \theta\|H-\mathrm{id}\|,
$$

for some uniform constant $\theta>0$. We then choose $\eta<\varepsilon /(C \theta)$ such that the ball centered at id and with radius $\eta$ in $G L(d, \mathbb{R})$ is contained in $\mathcal{O}$. Set $n_{2}=n_{3}(d, C, K, \eta)$.

Let $\left(A_{i}\right)$ be any sequence in $G L(d, \mathbb{R})$ satisfying $\left\|A_{i}\right\|,\left\|A_{i}^{-1}\right\|<K$ and any integer $n \geq n_{2}$. Let us consider the sequence $\left(H_{0}, \ldots H_{r-1}\right)$ and the integer $0 \leq k \leq n$ given by Proposition 5.4. By our assumptions each matrix $H_{i}$ belongs to $\mathcal{O}$ and can be associated to a diffeomorphism $h_{i}=h\left(H_{i}\right)$ by Lemma 5.5.

We define the cocycle $g: \mathbb{Z} \times \mathbb{R}^{d} \rightarrow \mathbb{Z} \times \mathbb{R}^{d}$ as follows.

$$
g(x)= \begin{cases}f^{i+1} \circ h_{i} \circ f^{-i}(x), & \text { on }\{i\} \times \mathbb{R}^{d} \text { with } 0 \leq i \leq r, \\ f^{n-i} \circ h_{i}^{-1} \circ f^{n-i-1}(x), & \text { on }\{n-i-1\} \times \mathbb{R}^{d} \text { with } 0 \leq i \leq r, \\ f(x) & \text { otherwise. }\end{cases}
$$

By construction, $g$ is a tidy perturbation of $f$ supported on $\bigcup_{i=0}^{n-1} f^{i}(\{0\} \times Q)$.

On the set $\{i\} \times \mathbb{R}^{d}$, for $0 \leq i \leq r$, the $C^{1}$ distance between $f$ and $g$ is bounded by

$$
d_{C^{1}}\left(h_{i}, \mathrm{id}\right) \leq \max \left\{\left\|A_{i}\right\|,\left\|A_{i}^{-1}\right\|\right\} \cdot e\left(A_{i-1} \cdots A_{0}\right) \cdot \theta \cdot\left\|H_{i}-\mathrm{id}\right\|<C \theta \eta<\varepsilon .
$$


The same estimate holds on $\{n-i-1\} \times \mathbb{R}^{d}$. Consequently the distance $d_{C^{1}}(f, g)$ is bounded by $\varepsilon$.

Since the map $h_{i}$ coincides with $H_{i}$ on $\delta Q$, it maps $\delta Q$ into itself. For each $0 \leq$ $i<r$, the map $g$ sends the set $f^{i}(\{0\} \times \delta Q)$ into the set $f^{i+1}(\{0\} \times \delta Q)$ and coincides with $\left(A_{i} \ldots A_{0}\right) H_{0}\left(A_{i} \ldots A_{0}\right)^{-1}$. It follows that on the set $g^{-k}(\{0\} \times \delta Q)$ the map $g^{n}$ is linear and coincides with the product $P=A_{-k+n-1} \cdots A_{0} H_{r-1} \cdots H_{0} A_{-1} \cdots A_{-k}$. In particular $\max \left\{\| D g^{n}\left(g^{-k}(x)\|\|, D g^{-n}\left(g^{n-k}(x)\right) \|\right\}\right.$ is larger than $K$ on $\{0\} \times \delta Q$.

\subsection{Huge versus bounded intermediary products}

We now come to the proof of Proposition 5.4. Let $d \geq 1, K>1$ and $C, \eta>0$ be given. We choose $e_{0}>0$ and $s \in(0,1)$ such that

$$
e_{0}>\eta^{-1}\left(K^{2}+1\right)^{2} \text { and } 2 e_{0} \frac{s}{1+s}<\eta .
$$

Next, we choose $n_{3}$ satisfying

$$
(1+s)^{n_{3} / 2-1}>K^{2}
$$

Let us consider $\left(A_{i}\right)$ and $n \geq n_{3}$ as in the statement of the proposition. We may assume that the products $A_{-k+n-1} \cdots A_{k}$ and their inverses have a norm bounded by $K$, since otherwise the conclusion of Proposition 5.4 holds already. Two cases are possible.

Huge intermediary products. We first assume that

$$
\text { There exists } i_{0} \in\{1, \ldots, n\} \text { such that } e\left(A_{i_{0}-1} \cdots A_{0}\right)>e_{0} \text {. }
$$

Decompose the linear map $A=A_{i_{0}-1} A_{i_{0}-2} \ldots A_{i_{0}-n}$ into a product $A=E F$, where $E=A_{i_{0}-1} \ldots A_{0}$ and $F=A_{-1} \ldots A_{i_{0}-n}$. By assumption we have $e(E)>e_{0}$, and $\mathcal{M}(A)=\left\|A^{-1}\right\|^{-1} \geq \frac{1}{K}$.

Let $u$ and $v$ be orthogonal unit vectors in $\mathbb{R}^{d}$ satisfying $\|E u\|=\|E\|$ and $\|E v\|=$ $\mathcal{M}(E)$. Then $\|E u\|=e(E)\|E v\|>e_{0}\|E v\|$.

Since $e_{0} \eta>\left(K^{2}+1\right)^{2}$, we can choose $t \in\left(\frac{K^{2}+1}{e_{0}}, \frac{\eta}{K^{2}+1}\right)$. Let $H \in G L(d, \mathbb{R})$ be a linear map satisfying $H(v)=v+t u$ and $\| H-$ id $\|=t$. Let $P=E H F$. We claim that the norm of $P$ is greater than $K$. To show this, we will use the following elementary fact:

Claim. Let $A, P \in G L(d, \mathbb{R})$. Suppose there exists a constant $\gamma>0$ and a nonzero vector $w \in \mathbb{R}^{d}$ such that $\|P w\|>\gamma\|A w\|$. Then $\|P\|>\gamma \mathcal{M}(A)$.

To apply this lemma, we set $w=F^{-1}(v)$, and calculate:

$$
\begin{gathered}
\|P w\|=\left\|E H F\left(F^{-1}(v)\right)\right\|=\|E H(v)\|=\|E(v+t u)\| \geq t\|E(u)\|-\|E(v)\| \\
>\left(t e_{0}-1\right)\|E(v)\|=\left(t e_{0}-1\right)\|A w\| .
\end{gathered}
$$


Applying the lemma with $\gamma=t e_{0}-1$, we see that

$$
\|P\|>\left(t e_{0}-1\right) \mathcal{M}(A) \geq \frac{\left(t e_{0}-1\right)}{K}>K,
$$

by our lower bound on $t$.

By setting $r=0$ and $H_{0}=H$, we obtain the first conclusion of the proposition. The second one is empty. Since $\left\|A_{n-1} \ldots A_{0}\right\|$ and $\left\|\left(A_{n-1} \ldots A_{0}\right)^{-1}\right\|$ are bounded by $K$, we obtain that $e\left(A_{n-1} \ldots A_{0}\right)<K^{2}$. As a consequence, we have

$$
\left(e\left(A_{n-1} \cdots A_{0}\right)+1\right)\left\|H_{0}-\mathrm{id}\right\| \leq\left(K^{2}+1\right) t<\eta,
$$

by our upper bound on $t$. This gives the last conclusion.

Bounded intermediary products. Assume, on the other hand, that

For any $i_{0} \in\{1, \ldots, n\}$ we have $e\left(A_{i_{0}-1} \cdots A_{0}\right) \leq e_{0}$.

Let $r=\left\lfloor\frac{n}{2}\right\rfloor$ and let $H \in G L(d, \mathbb{R})$ be the linear conformal dilation $H=(1+$ $s)^{-1} I$. Since this is a linear contraction, $H$ maps the standard cube $Q$ into itself. We define $H_{i}=H$ for any $i=0, \ldots, r-1$. Note that

$$
\left(e\left(A_{n-i-1} \cdots A_{0}\right)+e\left(A_{i-1} \cdots A_{0}\right)\right) \cdot\left\|H_{i}-\mathrm{id}\right\| \leq 2 e_{0} \frac{s}{1+s}<\eta,
$$

by our choice of $s$.

It is straightforward to check that for $k=n-r$, the product $P=$ $A_{-k+n-1} \cdots A_{0} H_{r-1} \cdots H_{0} A_{-1} \cdots A_{-k}$ satisfies

$$
\left\|P^{-1}\right\| \geq(1+s)^{r}\left\|A_{-k+n-1} \cdots A_{-k}\right\|>(1+s)^{n_{3} / 2-1} K^{-1}>K,
$$

by our choice of $n_{3}$. The conclusions of the proposition are thus satisfied.

\section{6 (UD) property: reduction to a perturbation result in a cube}

The aim of this section is to provide successive reductions for Theorem A. At the end we are led to a perturbation result (Proposition 6.6) for cocycles that produces an arbitrarily large variation of the jacobian along orbits in a cube. Many of the difficulties we meet in the proof of Theorem A come from the fact that we have not been able to create a large change in the jacobian of a linear cocycle, inside a cube, by a tidy perturbation.

\subsection{Reduction to a perturbation result in towers}

We show that Theorem B follows from a perturbation result that produces arbitrarily large distortion between a given orbit and the orbit of a wandering compact set.

Proposition 6.1. Consider a diffeomorphism $f$, a compact ball $\Delta$, an open set $U$, and a point $x$ of $M$ satisfying: 
- $f(\bar{U}) \subset U$;

- $\Delta \subset U \backslash f(\bar{U})$;

- the orbit of $x$ is disjoint from $\Delta$.

Then for any $K, \varepsilon>0$ there exists a diffeomorphism $g$ with $d_{C^{1}}(f, g)<\varepsilon$ having the following property: for all $y \in \Delta$, there exists $n \geq 1$ such that

$$
\left|\log \operatorname{det} D g^{n}(x)-\log \operatorname{det} D g^{n}(y)\right|>K .
$$

Moreover, $f=g$ on a neighborhood of the chain-recurrent set $C R(f)$.

Proof of Theorem A from Proposition 6.1. Let $\mathcal{X} \subset M$ be a countable dense set and let $\mathcal{K}=\left\{\Delta_{n}\right\}$ be a countable collection of compact balls in $M$ satisfying:

- $\operatorname{diam} \Delta_{n} \rightarrow 0$ as $n \rightarrow \infty$, and

- $\bigcup_{n \geq n_{1}} \Delta_{n}=M$ for all $n_{1} \geq 1$.

For $\Delta \in \mathcal{K}$, define the open subset of $\operatorname{Diff}^{1}(M)$ :

$$
\mathcal{O}_{\Delta}=\left\{f \in \operatorname{Diff}^{1}(M) \mid \exists \text { open set } U, f(\bar{U}) \subset U, \Delta \subset U \backslash f(\bar{U})\right\} .
$$

For $x \in \mathcal{X}$, define

$$
\mathcal{U}_{x, \Delta}=\left\{f \in \mathcal{O}_{\Delta} \mid \operatorname{orb}_{f}(x) \cap \Delta=\emptyset\right\} .
$$

Notice that $f \in \mathcal{U}_{x, \Delta}$ means that there is an open set $U$ such that $(\Delta, U, x)$ satisfies the hypotheses of Proposition 6.1.

The set $\mathcal{U}_{x, \Delta}$ is not open. The next lemma gives a simple criterion for $f$ to belong to its interior:

Lemma 6.2. Consider $f \in \mathcal{U}_{x, \Delta}$ and an open subset $U$ of $M$ with $f(\bar{U}) \subset U$, $\Delta \subset U \backslash f(\bar{U})$. Assume that the orbit of $x$ meets $U \backslash f(\bar{U})$. Then $f$ belongs to the interior of $\mathcal{U}_{x, \Delta}$ : the orbit of $x$ under any diffeomorphism $g$ sufficiently $C^{1}$-close to $f$ is disjoint from $\Delta$.

Proof. Consider $i \in \mathbb{Z}$ such $f^{i}(x) \in U \backslash \overline{f(U)}$. Such a number $i$ is unique, because $U \backslash \overline{f(U)}$ is the fundamental domain of an attracting region $U$ and hence is disjoint from all its iterates. Moreover, there is a neighborhood $\mathcal{U}$ of $f$ such that every $g \in \mathcal{U}$ satisfies:

- $g(\bar{U}) \subset U$,

- $\Delta \subset U \backslash \overline{g(U)}$,

- $g^{i}(x) \in U \backslash(\overline{g(U)} \cup \Delta)$.

This shows that the open neighborhood $\mathcal{U}$ of $f$ is contained in $\mathcal{U}_{x, \Delta}$.

In order to obtain a residual set, we must first produce a countable family of open and dense subsets of $\operatorname{Diff}^{1}(M)$. However the sets $\mathcal{U}_{x, \Delta}$ are neither open nor closed. The next lemma shows the way to bypass this difficulty: 
Lemma 6.3. The set $\operatorname{Int}\left(\mathcal{U}_{x, \Delta}\right) \cup \operatorname{Int}\left(\mathcal{O}_{\Delta} \backslash \mathcal{U}_{x, \Delta}\right)$ is open and dense in $\mathcal{O}_{\Delta}$.

Proof. The set $\operatorname{Int}\left(\mathcal{U}_{x, \Delta}\right) \cup \operatorname{Int}\left(\mathcal{O}_{\Delta} \backslash \mathcal{U}_{x, \Delta}\right)$ is open by definition; we just have to prove its density in $\mathcal{O}_{\Delta}$.

Fix $f \in \mathcal{O}_{\Delta}$. Then there exists an open set $U \subset M$ and an open neighborhood $\mathcal{U} \subset \mathcal{O}_{\Delta}$ of $f$ such that for $g \in \mathcal{U}$ we have $g(\bar{U}) \subset U$ and $\Delta \subset U \backslash g(\bar{U})$. Now fix $x \in X$. We will show that $\mathcal{U} \cap\left(\operatorname{Int}\left(\mathcal{U}_{x, \Delta}\right) \cup \operatorname{Int}\left(\mathcal{O}_{\Delta} \backslash \mathcal{U}_{x, \Delta}\right)\right)$ is dense in $\mathcal{U}$.

Let $\mathcal{V} \subset \mathcal{U}$ be the open subset of diffeomorphisms $g$ such that the orbit $\operatorname{orb}_{g}(x)$ meets $U \backslash g(\bar{U})$. Any diffeomorphism $g \in \mathcal{U} \backslash \mathcal{V}$ belongs to $\mathcal{U}_{x, \Delta}$ : the orbit of $x$ is disjoint from $U \backslash g(\bar{U})$, and so is disjoint a fortiori from $\Delta$. Let $\mathcal{W}_{0}=\mathcal{U} \backslash \overline{\mathcal{V}}$. By construction, $\mathcal{V} \cup \mathcal{W}_{0}$ is open and dense in $\mathcal{U}$, and $\mathcal{W}_{0} \subset \operatorname{Int}\left(\mathcal{U}_{x, \Delta}\right)$.

Lemma 6.2 asserts that $\mathcal{V}_{0}=\mathcal{V} \cap U_{x, \Delta}$ is open. Let $\mathcal{W}_{1}=\mathcal{V} \backslash \overline{\mathcal{V}_{0}}$. Then $\mathcal{W}_{1}$ is an open set contained in $\mathcal{O}_{\Delta} \backslash \mathcal{U}_{x, \Delta}$ and hence in $\operatorname{Int}\left(\mathcal{O}_{\Delta} \backslash \mathcal{U}_{x, \Delta}\right)$. Moreover, $\mathcal{V}_{0} \cup \mathcal{W}_{1}$ is open and dense in $\mathcal{V}$. We have thus shown that $\mathcal{V}_{0} \cup \mathcal{W}_{1} \cup \mathcal{W}_{0}$ is an open and dense subset of $\mathcal{U}$ contained in $\operatorname{Int}\left(\mathcal{U}_{x, \Delta}\right) \cup \operatorname{Int}\left(\mathcal{O}_{\Delta} \backslash \mathcal{U}_{x, \Delta}\right)$, ending the proof.

For $x \in \mathcal{X}, \Delta \in \mathcal{K}$ and any integer $K \in \mathbb{N}$, we define:

$$
\begin{aligned}
\mathcal{V}_{x, \Delta, K}=\left\{f \in \operatorname{Int}\left(\mathcal{U}_{x, \Delta}\right) \mid \forall y \in \Delta,\right. & \exists n \geq 1, \\
& \left.\left|\log \operatorname{det} D f^{n}(x)-\log \operatorname{det} D f^{n}(y)\right|>K\right\} .
\end{aligned}
$$

Note that $\mathcal{V}_{x, \Delta, K}$ is open in $\operatorname{Int}\left(\mathcal{U}_{x, \Delta}\right)$. Furthermore $\mathcal{V}_{x, \Delta, K}$ is dense $\operatorname{in} \operatorname{Int}\left(\mathcal{U}_{x, \Delta}\right)$, by Proposition 6.1. If follows that the set

$$
\mathcal{W}_{x, \Delta, K}=\mathcal{V}_{x, \Delta, K} \cup \operatorname{Int}\left(\mathcal{O}_{\Delta} \backslash \mathcal{U}_{x, \Delta}\right) \cup \operatorname{Int}\left(\operatorname{Diff}^{1}(M) \backslash \mathcal{O}_{\Delta}\right)
$$

is open and dense in $\operatorname{Diff}^{1}(M)$. Next, we set

$$
\mathcal{G}_{0}=\bigcap \mathcal{W}_{x, \Delta, K}
$$

where the intersection is taken over $x \in \mathcal{X}, \Delta \in \mathcal{K}$, and $K \in \mathbb{N}$. This set is residual in $\operatorname{Diff}^{1}(M)$. Finally, we take $\mathcal{G}$ to be the intersection of $\mathcal{G}_{0}$ with the residual set of $f \in \operatorname{Diff}^{1}(M)$ constructed in $[\mathrm{BC}]$ where the chain recurrent and nonwandering sets coincide.

Consider $f \in \mathcal{G}$. Fix $x \in \mathcal{X}$ and $y \in M \backslash \Omega(f)=M \backslash C R(f)$ such that $\operatorname{orb}(x) \cap \operatorname{orb}(y)=\emptyset$. Since $y$ is not chain recurrent, Conley theory implies that there exists an open set $U \subset M$ such that $f(\bar{U}) \subset U$ and $y \in U \backslash \overline{f(U)}$ (see Section 2.1). Observe that $\operatorname{orb}(x) \cap U \backslash \overline{f(U)}$ contains at most one point; it is distinct from $y$ by assumption. Thus any compact set $\Delta \in \mathcal{K}$ containing $y$ and with sufficiently small diameter satisfies

- $\Delta \subset U \backslash f(\bar{U})$,

- $\operatorname{orb}(x) \cap \Delta=\emptyset$.

We fix such a compact set $\Delta \in \mathcal{K}$. This implies that $f \in \mathcal{U}_{x, \Delta}$. Since $f \in \mathcal{G}_{0}$, the definition of $\mathcal{G}_{0}$ implies that for every $K \in \mathbb{N}$, we have $f \in \mathcal{W}_{x, \Delta, K}$; since $f \in U_{x, \Delta}$, we must have $f \in \mathcal{V}_{x, \Delta, K}$. This means that, for every $K$, we have for some $n \geq 1$

$$
\left|\log \operatorname{det} D f^{n}(x)-\log \operatorname{det} D f^{n}(y)\right|>K .
$$

Hence $f$ satisfies property (UD) on the nonwandering set. 


\subsection{Localization of the perturbation}

Here we reduce Proposition 6.1 to the case where $\Delta$ has small diameter.

We use the following notation. If $X \subset M$ is a compact set and $\delta>0$ then $U_{\delta}(X)$ denotes the $\delta$-neighborhood of $X: U_{\delta}(X)=\{y \in M \mid d(y, X)<\delta\}$.

Proposition 6.4. For any $d \geq 1$ and $C, K, \varepsilon>0$, there exists $n_{0}=n_{0}(C, K, \varepsilon)$ with the following property.

For any diffeomorphism $f$ of a d-dimensional manifold $M$ satisfying $\|D f\|,\left\|D f^{-1}\right\|<C$, there exists $\rho_{0}=\rho_{0}(d, C, K, \varepsilon)$ such that for any $\eta>0$, any compact set $\Delta \subset M$ and $x \in M$ satisfying:

- $\operatorname{diam}(\Delta)<\rho_{0}$,

- $\Delta$ is disjoint from its first $n_{0}$ iterates $\left\{f^{i}(\Delta): 1 \leq i \leq n_{0}\right\}$,

- $\operatorname{orb}(x) \cap \Delta=\emptyset$,

there exists a diffeomorphism $g \in \operatorname{Diff}^{1}(M)$ such that

- $d_{C^{1}}(f, g)<\varepsilon$,

- $d_{C^{0}}(f, g)<\eta$,

- for all $y \in \Delta$, there exists an integer $n \in\left\{1, \ldots, n_{0}\right\}$ such that:

$$
\left|\log \operatorname{det} D g^{n}(x)-\log \operatorname{det} D g^{n}(y)\right|>K .
$$

Moreover, $f=g$ on the complement of $U_{\eta}\left(\bigcup_{i=0}^{n_{0}-1} f^{i}(\Delta)\right)$.

Proof of Proposition 6.1 from Proposition 6.4. Let $f, \Delta, U, x$ be as in the statement of Proposition 6.1 Choose $n_{0}=n_{0}(d, C, 2 K, \varepsilon)$ and $\rho_{0}=\rho_{0}(d, C, K, \varepsilon)$ according to Proposition 6.4. We set $N=2^{d} n_{0}$.

Cover $\Delta$ by a finite collection $\mathcal{F}$ of compact sets satisfying:

- $\Delta \subset \bigcup_{D \in \mathcal{F}} \operatorname{Int}(D) \subset U \backslash f(\bar{U})$, so that for each $D \in \mathcal{F}$ the iterates $D, f(D), f^{2}(D), \ldots$ are pairwise disjoint;

- $\operatorname{orb}(x) \cap \bigcup_{D \in \mathcal{F}} D=\emptyset$;

- $\operatorname{diam}\left(f^{i}(D)\right)<\rho_{0}$, for all $D \in \mathcal{F}$ and $i \in\{0, \ldots, N\}$;

- $\mathcal{F}=\mathcal{F}_{0} \cup \cdots \cup \mathcal{F}_{2^{d}-1}$, where $\mathcal{F}_{i} \cap \mathcal{F}_{j}=\emptyset$ for $i \neq j$ and the elements of $\mathcal{F}_{j}$ are pairwise disjoint for each $j$.

One can obtain $\mathcal{F}$ by tiling by arbitrarily small cubes the compact ball $\Delta$.

Let $\lambda>0$ be the Lebesgue number of the cover $\mathcal{F}$. For any $\eta>0$ we define an increasing sequence $\left(a_{\eta}(n)\right)$ by the inductive formula:

$$
a_{\eta}(0)=0 ; \quad a_{\eta}(n+1)=C a_{\eta}(n)+\eta .
$$

Note that for $n \geq 0$ fixed, we have $a_{\eta}(n) \rightarrow 0$ as $\eta \rightarrow 0$.

Let $\eta>0$ be small such that: 
- for each $D \in \mathcal{F}$ and $0 \leq i<N-1$, the $\eta$-neighborhood $U_{\eta}\left(f^{i}(D)\right)$ is contained in $f^{i}(U) \backslash \overline{f^{i+1}(U)}$; in particular the sets $U_{\eta}(D), \ldots, U_{\eta}\left(f^{N-1}(D)\right)$ are pairwise disjoint;

- the orbit $\operatorname{orb}(x)$ is disjoint from the union $\bigcup_{i \in\{0, \ldots, N\}} U_{\eta}\left(f^{i}(D)\right)$;

- for each distinct $(j, D)$ and $\left(j^{\prime}, D^{\prime}\right)$ with $j, j^{\prime} \in\left\{0, \ldots, 2^{d}-1\right\}$ and $D \in \mathcal{F}_{j}$, $D^{\prime} \in \mathcal{F}_{j^{\prime}}$, we have for all $k, k^{\prime} \in\left\{0, \ldots, n_{0}-1\right\}$,

$$
U_{\eta}\left(f^{n_{0} j+k}(D)\right) \cap U_{\eta}\left(f^{n_{0} j^{\prime}+k^{\prime}}\left(D^{\prime}\right)\right)=\emptyset ;
$$

- $a_{\eta}(N)<C^{-N} \lambda$.

For $j \in\left\{0, \ldots, 2^{d}-1\right\}$ and $D \in \mathcal{F}_{j}$, the set $f^{n_{0} j}(D)$ and the point $f^{n_{0} j}(x)$ satisfy the hypotheses of Proposition 6.4. We obtain a perturbation of $f$ supported on the $\eta$-neighborhood of $\bigcup_{k \in\left\{0, \ldots, n_{0}-1\right\}} f^{n_{0} j+k}(D)$; by our choice of $\eta$, any two such perturbations for distinct choices of $(j, D)$ will be disjointly supported. Hence, applying Proposition 6.4 over all pairs $(j, D)$ with $j \in\left\{0, \ldots, 2^{d}-1\right\}$ and $\left.D \in \mathcal{F}_{j}\right\}$, we obtain a perturbation $g$ with the following properties:

1. $d_{C^{1}}(f, g)<\varepsilon$

2. $d_{C^{0}}(f, g)<\eta$;

3. $g=f$ on $M \backslash U_{\eta}\left(\bigcup_{k=0}^{N-1} f^{k}(D)\right)$;

4. for each $j \in\left\{0, \ldots, 2^{d}-1\right\}$ and each $y \in \bigcup_{D \in \mathcal{F}_{j}} D$, there exists $n \in\left\{1, \ldots, n_{0}\right\}$ such that:

$$
\left|\log \operatorname{det} D g^{n}\left(f^{n_{0} j} x\right)-\log \operatorname{det} D g^{n}\left(f^{n_{0} j} y\right)\right|>2 K \text {. }
$$

We now prove the large derivative formula. We fix $y \in \Delta$.

Claim. There exist $j \in\left\{0, \ldots, 2^{d}-1\right\}$ and $D \in \mathcal{F}_{j}$ such that $g^{n_{0} j}(y) \in f^{n_{0} j}(D)$.

Proof. Choose $D \in \mathcal{F}$ such that the ball $B(y, \lambda)$ is contained in $D$ and fix $j \in$ $\left\{1, \ldots, 2^{d}\right\}$ such that $D \in \mathcal{F}_{j}$. This implies that $B\left(f^{k}(y), C^{-k} \lambda\right) \subset f^{k}(D)$, for all $k \in\{0, \ldots, N-1\}$. Note that

$$
\begin{aligned}
d\left(f^{k+1}(y), g^{k+1}(y)\right) & \leq d\left(f^{k+1}(y), f\left(g^{k}(y)\right)\right)+d\left(f\left(g^{k}(y)\right), g^{k+1}(y)\right) \\
& \leq C d\left(f^{k}(y), g^{k}(y)\right)+\eta
\end{aligned}
$$

which implies, by Property 2 above and our choice of $\lambda$, that for any $k \in\{0, \ldots, N-$ 1\} we have:

$$
d\left(f^{k}(y), g^{k}(y)\right) \leq a_{\eta}(k)<C^{-N} \lambda .
$$

We conclude that $g^{n_{0} j}(y) \in f^{n_{0} j}(D)$. 
Since the orbit of $x$ is disjoint from the support of the perturbation, we have that $f^{n_{0} j}(x)=g^{n_{0} j}(x)$, for all $j \in\left\{1, \ldots, 2^{d}-1\right\}$. From these properties and from (11), there exist $j \in\left\{0, \ldots, 2^{d}-1\right\}$ and $n \in\left\{1, \ldots, n_{0}\right\}$ such that:

$$
\left|\log \operatorname{det} D g^{n}\left(g^{n_{0} j} x\right)-\log \operatorname{det} D g^{n}\left(g^{n_{0} j} y\right)\right|>2 K .
$$

The fact that $D g^{n+n_{0} j}=D g^{n} \circ D g^{n_{0} j}$ implies that one of these two cases holds:

- either $\left|\log \operatorname{det} D g^{n_{0} j}(x)-\log \operatorname{det} D g^{n_{0} j}(y)\right|>K$,

- or $\left|\log \operatorname{det} D g^{n+n_{0} j}(x)-\log \operatorname{det} D g^{n+n_{0} j}(y)\right|>K$.

In any case, there exists $n \in\{1, \ldots, N\}$ such that the required estimate $\left|\log \operatorname{det} D g^{n}(x)-\log \operatorname{det} D g^{n}(y)\right|>K$ holds.

By construction the support of the perturbation is contained in a finite number of iterates of $U \backslash \overline{f(U)}$; the iterates of $U \backslash \overline{f(U)}$ for $f$ and $g$ hence coincide. This implies that $C R(f)=C R(g)$.

\subsection{Reduction to cocycles}

Proposition 6.4 is a consequence of the following result about cocycles.

Proposition 6.5. For any $d \geq 1$ and any $C, K, \varepsilon>0$, there exists $n_{1}=$ $n_{1}(d, C, K, \varepsilon) \geq 1$ with the following property.

Consider any sequence $\left(A_{i}\right)$ in $G L(d, \mathbb{R})$ with $\|A\|,,\left\|A^{-1}\right\|<C$ and the associated cocycle $f$. Then, for any open set $U \subset \mathbb{R}^{d}$, for any compact set $\Delta \subset U$ and for any $\eta>0$, there exists a diffeomorphism $g$ of $\mathbb{Z} \times \mathbb{R}^{d}$ such that:

- $d_{C^{1}}(f, g)<\varepsilon$,

- $d_{C^{0}}(f, g)<\eta$,

- $g=f$ on the complement of $\bigcup_{i=0}^{2 n_{1}-1} f^{i}(\{0\} \times U)$,

- for all $y \in\{0\} \times \Delta$, there exists $n \in\left\{1, \ldots, n_{1}\right\}$ such that

$$
\left|\log \operatorname{det} D f^{n}(y)-\log \operatorname{det} D g^{n}(y)\right|>K .
$$

Proof of Proposition 6.4 from Proposition 6.5. Fix $d, C, K, \varepsilon>0$, choose $0<\tilde{\varepsilon}<\varepsilon, K_{0}>2 K+8 \log 2$ and set $n_{0}=2 n_{1}\left(d, C, K_{0}, \tilde{\varepsilon}\right)$.

Let $f: M \rightarrow M$ be a diffeomorphism such that $\|D f\|,\left\|D f^{-1}\right\|<C$ and let $\delta>0$ be the constant associated to $f, \tilde{\varepsilon}, \varepsilon$, and $n_{0}$ by Lemma 3.14. Fix $\rho_{0} \in\left(0, \frac{\delta}{2} C^{-n_{1}}\right)$.

Consider $\eta, \Delta$ and $x$ as in the statement of Proposition 6.4. We fix an open neighborhood $U$ of $\Delta$ such that:

- $\operatorname{diam}(U)<\rho_{0}$; in particular $\operatorname{diam}\left(f^{i}(U)\right)<\delta$ for all $i \in\left\{0, \ldots, n_{1}\right\}$;

- $U$ is disjoint from its first $n_{0}$ iterates;

- $f^{i}(x) \notin U$ for $i \in\left\{0, \ldots, n_{0}\right\}$. 
Fix a point $z_{0} \in \Delta$ and let $\tilde{f}$ denote the linear cocycle induced by the derivative $D f$ along the orbit of $z_{0}$.

By Lemma 3.14, there are diffeomorphisms $\Psi_{i}: f^{i}(U) \rightarrow \tilde{U}_{i} \subset T_{f^{i}\left(z_{0}\right)} M$ for $i \in\left\{0, \ldots, n_{1}\right\}$, which conjugate $f$ to $\tilde{f}$ : for every $z \in f^{i}(U)$ we have $\tilde{f}\left(\Psi_{i}(z)\right)=$ $\Psi_{i+1}(f(z))$. Moreover,

- $\left\|D \Psi_{i}\right\|,\left\|D \Psi_{i}^{-1}\right\|,\left|\operatorname{det} D \Psi_{i}\right|$ and $\left|\operatorname{det} D \Psi_{i}^{-1}\right|$ are bounded by 2 ;

- any $\tilde{\varepsilon}$-perturbation $\tilde{g}$ of $\tilde{f}$ with support in $\bigcup_{i=0}^{n_{0}-1} \tilde{U}_{i}$ induces a diffeomorphism $g$ which is a $\varepsilon$-perturbation of $f$ supported on $\bigcup_{i=0}^{n_{0}-1} f^{i}(U)$ through a conjugacy by the diffeomorphisms $\Psi_{i}$.

We denote by $\Psi: \bigcup_{i=0}^{n_{0}} f^{i}(U) \rightarrow \bigcup_{i=0}^{n_{0}} \tilde{U}_{i}$ the diffeomorphism that is equal to $\Psi_{i}$ on $f^{i}(U)$.

We now apply Proposition 6.5 to obtain a $\tilde{\varepsilon}$-perturbation $\tilde{g}$ of $\tilde{f}$ supported in $\bigcup_{i=0}^{n_{0}-1} \tilde{U}_{i}$ such that for every $y \in \Psi_{0}(\Delta)$ we have:

- $d_{C^{0}}(\tilde{f}, \tilde{g})<\frac{\eta}{2}$,

- $\tilde{g}=\tilde{f}$ on the complement of $\bigcup_{i=0}^{n_{0}-1} \tilde{U}_{i}$,

- for all $y \in \Psi_{0}(\Delta)$ there exists $n \in\left\{1, \ldots, n_{0}\right\}$ such that

$$
\left|\log \operatorname{det} D \tilde{f}^{n}(y)-\log \operatorname{det} D \tilde{g}^{n}(y)\right|>K_{0} .
$$

Let $g$ be the corresponding $\varepsilon$-perturbation of $f$. Since $\Psi$ satisfies $\left\|D \Psi^{-1}\right\| \leq 2$, we obtain that

$$
d_{C^{0}}(f, g)<2 d_{C^{0}}(\tilde{f}, \tilde{g})<\eta .
$$

Furthermore, for each $z \in \Delta$, there exists $n \in\left\{1, \ldots, n_{0}\right\}$ such that

$$
\left|\log \operatorname{det} D f^{n}(z)-\log \operatorname{det} D g^{n}(z)\right|>K_{0}-4 \log 2 .
$$

For $n \in\left\{0, \ldots, n_{0}-1\right\}$, the maps $f^{n}$ and $D f^{n}\left(z_{0}\right)$ are conjugate on $\Delta$ by the diffeomorphism $\Psi$. Since $\|\operatorname{det} D \Psi\| \in\left[\frac{1}{2}, 2\right]$, this implies that, for every $z \in \Delta$, we have $\left|\log \operatorname{det} D f^{n}\left(z_{0}\right)-\log \operatorname{det} D f^{n}(z)\right| \leq 2 \log 2$. If there exists $n \in\left\{0, \ldots, n_{0}-1\right\}$ such that $\left|\log \operatorname{det} D f^{n}\left(z_{0}\right)-\log \operatorname{det} D f^{n}(x)\right|>K+2 \log 2$, then we do not perturb $f$ : in this case, every point $z \in \Delta$ satisfies $\left|\log \operatorname{det} D f^{n}(z)-\log \operatorname{det} D f^{n}(x)\right|>K$ as required. Hence we may assume that for every $n \in\left\{0, \ldots, n_{0}-1\right\}$ and every $z \in \Delta$ we have

$$
\left|\log \operatorname{det} D f^{n}(z)-\log \operatorname{det} D f^{n}(x)\right| \leq K+2 \log 2 .
$$

Notice that the support of the perturbation $g$ is disjoint from the set $\left\{x, f(x), \ldots, f^{n_{0}}(x)\right\}$, so that $D f^{n}(x)=D g^{n}(x)$ for $n \in\left\{0, \ldots, n_{0}\right\}$. Inequality (12) implies that for every $z \in \Delta$ there exists $n \in\left\{1, \ldots n_{0}\right\}$ such that

$$
\begin{aligned}
\left|\log \frac{\operatorname{det} D g^{n}(x)}{\operatorname{det} D g^{n}(z)}\right| & >\left|\log \frac{\operatorname{det} D f^{n}(z)}{\operatorname{det} D g^{n}(z)}\right|-\left|\log \frac{\operatorname{det} D f^{n}(x)}{\operatorname{det} D f^{n}(z)}\right| \\
& >K_{0}-4 \log 2-K-4 \log 2>K .
\end{aligned}
$$

This completes the proof of Proposition 6.4, assuming Proposition 6.5. 


\subsection{Reduction to a perturbation result in a cube}

We now reduce Proposition 6.5 to the case where $U$ is the interior of a cube $Q_{\mu}$, which has to be chosen to be very thin along one coordinate, and $\Delta$ is a smaller cube $\theta Q_{\mu}$.

We thus consider the space $\mathbb{R}^{d}$ as a product $\mathbb{R}^{d-1} \times \mathbb{R}$ and denote by $(v, z)$ its coordinates. For a constant $\mu>0$, let $E_{\mu}$ be the linear isomorphism of $\mathbb{R}^{d}$ defined by $E_{\mu}(v, z)=(v, \mu z)$. The image of the standard cube $Q=[-1,1]^{d}$ by $E_{\mu}$ will be denoted by $Q_{\mu}=[-1,1]^{d-1} \times[-\mu, \mu]$.

Proposition 6.6. For any $d \geq 1, C, K, \varepsilon>0$ and $\theta \in(0,1)$, there exists $n_{2}=$ $n_{2}(d, C, K, \varepsilon, \theta) \geq 1$ and for any $\eta>0$ there exists $\mu_{0}=\mu_{0}(d, C, K, \varepsilon, \theta, \eta)>0$ with the following property.

Consider any sequence $\left(A_{i}\right)$ in $G L(d, \mathbb{R})$ with $\left\|A_{i}\right\|,\left\|A_{i}^{-1}\right\|<C$ and the associated linear cocycle $f$. Consider any $\mu \in\left(0, \mu_{0}\right)$ and $j \in\left\{0, \ldots, 2^{d}-1\right\}$. Then there exists a diffeomorphism $g$ of $\mathbb{Z} \times \mathbb{R}^{d}$ such that:

- $d_{C^{1}}(f, g)<\varepsilon$;

- $g=f$ on the complement of $\bigcup_{i=2 j n_{2}}^{2(j+1) n_{2}-1} f^{i}\left(\{0\} \times Q_{\mu}\right)$;

- for all $x \in\{0\} \times \theta Q_{\mu}$,

$$
\left|\log \operatorname{det} D f^{n_{2}}\left(f^{2 j n_{2}}(x)\right)-\log \operatorname{det} D g^{n_{2}}\left(f^{2 j n_{2}}(x)\right)\right|>K \text {; }
$$

- on $\{0\} \times \mathbb{R}^{d}$, we have:

$$
d_{\text {unif }}\left(\mathrm{id}, E_{\mu}^{-1} f^{-2\left(j+1 n_{2}\right)} g^{2(j+1) n_{2}} E_{\left.\mu\right|_{\{0\} \times \mathbb{R}^{d}}}\right)<\eta .
$$

Proof of Proposition 6.5 from Proposition 6.6. The proof is partly similar to the proof of Proposition 5.1. Let $C, K, \varepsilon>0$ be given. We fix $\theta=\frac{9}{10}$ and set

$$
n_{1}(d, C, K, \varepsilon)=2^{d+1} n_{2}(C, 2 K, \varepsilon, \theta) \geq 1 .
$$

Consider $\left(A_{i}\right), \Delta, U, \eta$ as in the statement of Proposition 6.5. Up to rescaling by homothety, we may assume that:

$$
\begin{aligned}
& C^{2^{d+1} n_{1}} 2 \theta^{-1} \sqrt{d}<d\left(\Delta, \mathbb{R}^{d} \backslash U\right), \\
& C^{2^{d+1} n_{1}} 2 \theta^{-1} \sqrt{d}<\eta
\end{aligned}
$$

Indeed let us consider some $a \in(0,1)$ and the homothety $h_{\frac{1}{a}}$ of $\mathbb{R}^{d}$ with ratio $\frac{1}{a}$. If $g_{0}$ is a perturbation of $f$ satisfying the conclusions of Proposition 6.5 for $C, K, \varepsilon, h_{\frac{1}{a}}(\Delta), h_{\frac{1}{a}}(U)$ and $\frac{\eta}{a}$, then $g=h_{a} g_{0} h_{\frac{1}{a}}$ is a perturbation of $f$ satisfying the conclusions of Proposition 6.5 for $C, K, \varepsilon, \Delta, \stackrel{a}{U}$ and $\eta$. Hence we may assume that the estimates (14) and (15) hold by choosing $a$ small enough.

In order to apply Proposition 6.6, we tile the set $U$. The tiling we use has the same structure as the tiling used in Proposition 5.1, producing $2^{d}$ disjoint families 

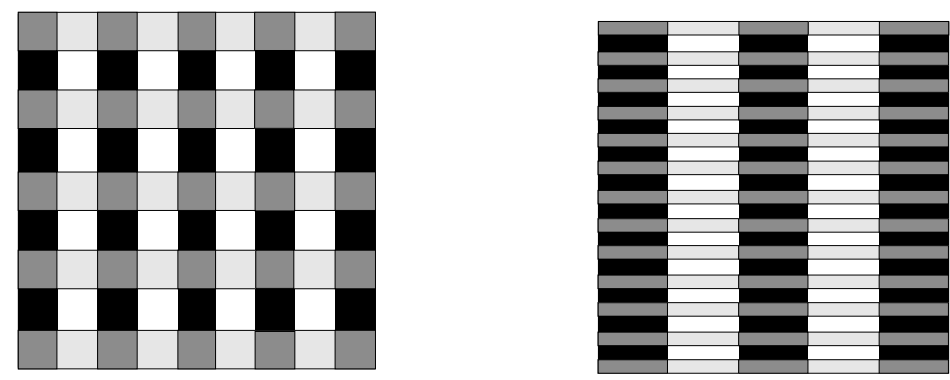

Figure 1: Tiling of the plane used in (LD) perturbation theorem (left) and (UD) perturbation theorem (right). The different shadings correspond to the partition into $2^{d}=4$ families.

of tiles, but the geometry is different (see Figure 1). We first consider the regular tilings $\mathcal{T}$ and $\tilde{\mathcal{T}}$ of $\mathbb{R}^{d}$ by the cubes

$$
\begin{aligned}
& Q_{i_{1}, \ldots, i_{d}}=\left[-\theta^{-1}, \theta^{-1}\right]^{d}+\left(i_{1}, \ldots, i_{d}\right), \\
& \tilde{Q}_{i_{1}, \ldots, i_{d}}=\left[-\theta^{-2}, \theta^{-2}\right]^{d}+\left(i_{1}, \ldots, i_{d}\right),
\end{aligned}
$$

respectively, where $\left(i_{1}, \ldots, i_{d}\right) \in \mathbb{Z}^{d}$. Let $\lambda$ be the Lebesgue number of the cover $\mathcal{T}$ of $\mathbb{R}^{d}$. We choose $\eta_{0}>0$ smaller than $2^{-d} \lambda$.

Now fix $\mu \in(0,1)$ smaller than $\mu_{0}\left(C, 2 K, \varepsilon, \theta, \eta_{0}\right)$ and denote by $\mathcal{T}_{\mu}$ and $\tilde{\mathcal{T}}_{\mu}$ the images of the tilings $\mathcal{T}$ and $\tilde{\mathcal{T}}$ by $E_{\mu}$. These are the tilings of $\mathbb{R}^{d}$ by the cubes

$$
\begin{aligned}
& Q_{\mu,\left(i_{1}, \ldots, i_{d}\right)}=\left[-\theta^{-1}, \theta^{-1}\right]^{d-1} \times\left[-\theta^{-1} \mu, \theta^{-1} \mu\right]+\left(i_{1}, \ldots, i_{d-1}, \mu i_{d}\right), \\
& \tilde{Q}_{\mu,\left(i_{1}, \ldots, i_{d}\right)}=\left[-\theta^{-2}, \theta^{-2}\right]^{d-1} \times\left[-\theta^{-2} \mu, \theta^{-2} \mu\right]+\left(i_{1}, \ldots, i_{d-1}, \mu i_{d}\right),
\end{aligned}
$$

respectively. By our assumption (14), any cube $Q_{\mu,\left(i_{1}, \ldots, i_{d}\right)}$ such that $\tilde{Q}_{\mu,\left(i_{1}, \ldots, i_{d}\right)}$ intersects the compact set $\Delta$ is contained in $U$. We denote by $\Gamma_{\mu}$ the family of cubes $Q_{\mu,\left(i_{1}, \ldots, i_{d}\right)}$ such that $\tilde{Q}_{\mu,\left(i_{1}, \ldots, i_{d}\right)}$ intersects $\Delta$.

We next consider the partition $\Gamma_{\mu}=\bigcup_{\ell=0}^{2^{d}-1} \Gamma_{\mu, \ell}$ such that for pair of any elements $Q, Q^{\prime}$ in $\Gamma_{\mu, \ell}$, the enlarged cubes $\tilde{Q}, \tilde{Q}^{\prime}$ are disjoint. (As in Section 5.2 we set $Q_{\mu,\left(i_{1}, \ldots, i_{d}\right)} \in \Gamma_{\mu, \ell}$ if $\ell=\sum_{j=1}^{d} \alpha_{j} 2^{j-1}$ where $\alpha_{j}=0$ if $i_{j}$ is even and $\alpha_{j}=1$ if $i_{j}$ is odd).

For each cube $Q \in \Gamma_{\mu, \ell}$, we also introduce the time interval $I_{\ell}=$ $\left\{2 n_{2} \ell, \ldots, 2 n_{2}(\ell+1)-1\right\}$. Proposition 6.6 produces a perturbation $g_{Q}$ supported on the union

$$
W_{Q}=\bigcup_{i \in I_{\ell}} f^{i}(\{0\} \times \tilde{Q})
$$

The construction of the famillies $\Gamma_{\mu, \ell}$ and of the intervals $I_{\ell}$ implies that $W_{Q}$ and $W_{Q^{\prime}}$ are disjoint if $Q, Q^{\prime} \in \Gamma_{\mu}$ are distinct. We finally define $g$ by $g_{Q}$ on $W_{Q}$, for $Q \in \Gamma_{\mu, \ell}$, and $g=f$ elsewhere. In particular, we have $g=f$ on the complement of $\bigcup_{i=0}^{n_{1}-1} f^{i}(\{0\} \times U)$. The perturbation $g$ is supported in a disjoint union of images 
$f^{i}\left(\{0\} \times \tilde{Q}_{\mu,\left(i_{1}, \ldots, i_{d}\right)}\right)$ for $i \in\left\{0, \ldots, 2^{d} n_{1}-1\right\}$ and by (15) satisfies $d_{C^{0}}(f, g)<\eta$. Each perturbation $g_{Q}$ satisfies $d_{C^{1}}\left(g_{Q}, f\right)<\varepsilon$, and hence $d_{C^{1}}(g, f)<\varepsilon$ holds. It remains to prove the last part of the conclusion of the proposition.

Claim. For every $y \in\{0\} \times \Delta$, there exist $\ell \in\left\{0, \ldots, 2^{d}-1\right\}$ and $Q \in \Gamma_{\ell}$ such that $g_{\eta}^{2 n_{2} \ell}(y) \in f^{2 n_{2} \ell}(Q)$.

Proof. By definition of $\lambda$, the ball $B\left(E_{\mu}^{-1}(y), \lambda\right)$ is contained in some cube $Q_{\left(i_{1}, \ldots, i_{d}\right)}$. The cube $Q=Q_{\mu,\left(i_{1}, \ldots, i_{d}\right)}=E_{\mu}\left(Q_{\left(i_{1}, \ldots, i_{d}\right)}\right)$ contains $y \in \Delta$ and hence belongs to $\Gamma_{\mu, \ell}$. Let $\ell \in\left\{0, \ldots, 2^{d}-1\right\}$ be such that $Q_{\mu,\left(i_{1}, \ldots, i_{d}\right)} \in \Gamma_{\mu, \ell}$.

Since $d_{\text {unif }}\left(\mathrm{id}, E_{\mu}^{-1} f^{-2 n_{2}(j+1)} g_{Q}^{2 n_{2}} f^{2 n_{2}(j)} E_{\mu}\right)<\eta_{0}$ for each $j \in\left\{0, \ldots, 2^{d}-1\right\}$, we obtain the bound

$$
\begin{aligned}
d_{\text {unif }}\left(\mathrm{id}, E_{\mu}^{-1} f^{-2 n_{2} \ell} g^{2 n_{2} \ell} E_{\mu}\right) & \leq \sum_{j=0}^{\ell-1} d_{\text {unif }}\left(\mathrm{id}, E_{\mu}^{-1} f^{-2 n_{2}(j+1)} g^{2 n_{2}} f^{2 n_{2}(j)} E_{\mu}\right) \\
& <2^{\ell} \eta_{0} \leq 2^{d} \eta_{0}<\lambda
\end{aligned}
$$

by our choice of $\eta_{0}$.

By our estimate above, the point $E_{\mu}^{-1} f^{-2 n_{2} \ell} g^{2 n_{2} \ell} E_{\mu}\left(E_{\mu}^{-1}(y)\right)$ belongs to the ball $B\left(E_{\mu}^{-1}(y), \lambda\right)$ and hence to $Q_{\left(i_{1}, \ldots, i_{d}\right)}$. This proves that $f^{2 n_{2} \ell}(Q)$ contains the point $g^{2 n_{2} \ell}(y)$ as required.

In order to conclude, we fix a point $y \in\{0\} \times \Delta$ and $\ell \in\left\{0, \ldots, 2^{d}-1\right\}, Q \in \Gamma_{\ell}$ such that $g_{\eta}^{2 n_{2} \ell}(y) \in f^{2 n_{2} \ell}(Q)$. Note that if

$$
\left|\log \operatorname{det} D f^{2 n_{2} \ell}(y)-\log \operatorname{det} D g^{2 n_{2} \ell}(y)\right|>K,
$$

then the last conclusion of the proposition already holds for $y$.

Otherwise, since $g^{2 n_{2} \ell}(y)$ belongs to $f^{2 n_{2} \ell}(Q)$, we have

$$
D g^{2 n_{2}}\left(g^{2 n_{2} \ell}(y)\right)=D g_{Q}^{2 n_{2}}\left(g^{2 n_{2} \ell}(y)\right) ;
$$

since $f$ is a linear cocycle, we obtain

$$
\log \operatorname{det} D f^{2 n_{2}}\left(g^{2 n_{2} \ell}(y)\right)=\log \operatorname{det} D f^{2 n_{2}}\left(f^{2 n_{2} \ell}(y)\right) .
$$

Thus the property satisfied by $g_{Q}$ implies that

$$
\left|\log \operatorname{det} D f^{2 n_{2}}\left(f^{2 n_{2} \ell}(y)\right)-\log \operatorname{det} D g^{2 n_{2}}\left(g^{2 n_{2} \ell}(y)\right)\right|>2 K .
$$

Using the fact that

$$
\left|\log \operatorname{det} D f^{2 n_{2} \ell}(y)-\log \operatorname{det} D g^{2 n_{2} \ell}(y)\right| \leq K,
$$

we then obtain that

$$
\left|\log \operatorname{det} D f^{2 n_{2}(\ell+1)}(y)-\log \operatorname{det} D g^{2 n_{2}(\ell+1)}(y)\right|>K .
$$

This gives again the last conclusion of the proposition and concludes the proof. 


\section{Almost tidy perturbation in a cube}

The aim of this section is to give the proof of Proposition 6.6, which finishes the proof of Theorem A and hence of the Main Theorem.

This proposition is very close in spirit to the original idea of C. Pugh for the famous $C^{1}$-closing lemma. Pugh wanted to perturb the orbit of a point $x$ in a cube in such a way that it exits the support of the perturbation through the orbit of another given point $y$. Pugh noticed that, at each time, one has much more freedom to perturb the orbits "in the direction of the smallest dimension of the image of the cube". As he needed to perform a perturbation in arbitrary directions, a linear algebra lemma allowed him to choose the pattern of the cube such that each direction would be at some time the smallest one.

Here we just want to perform a perturbation that modifies the jacobian: hence we can do it by a perturbation "in an arbitrary direction". However, in principle our perturbations have to be tidy, or very close to tidy. For this reason the support of the perturbation will have length $2 n_{2}$ : we obtain the perturbation of the jacobian during the first $n_{2}$ iterates, and then try to remove the perturbation during the last $n_{2}$ iterates. For this reason, we need that the smallest dimension of the image of our cube corresponds to the same direction, all along the time support of the perturbation. This is obtained by choosing a pattern of our cubes having one direction much smaller than the others.

An additional difficulty comes from the fact that we were not able to obtain a tidy perturbation. After several iterations, the thin edges of the cube could become strongly sheared above its base; for this reason, it was not possible to remove the perturbation in a neighborhood of these edges. By choosing the cubes's height small enough, we are able to remove the perturbation in a rectified cube which differs from the sheared one only in a small region (see Figure 2). This "almost tidy perturbation" turns out to be sufficient for our purposes.

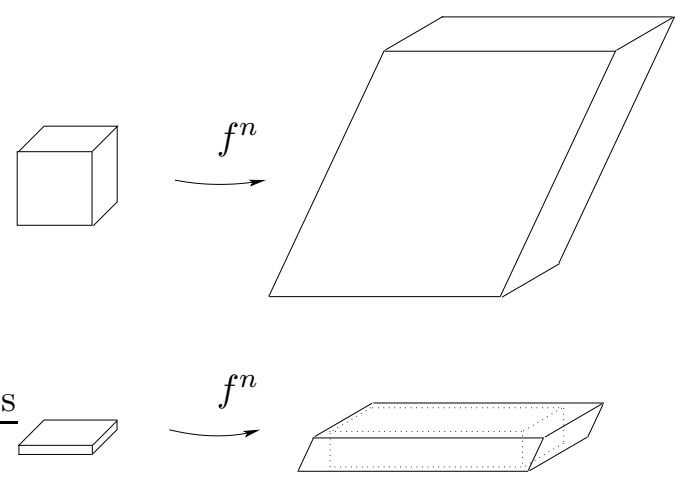

Figure 2: The image of a cube under many iterates of a linear cocycle can be quite distorted (top). The image of a wafer, on the other hand, stays flat and wafer-like (bottom). 


\subsection{Choice of $n_{2}$}

Given $d \geq 1$ and $\theta \in(0,1)$, we fix $\theta_{0}=\theta^{\frac{1}{3}}$ and choose:

- a smooth function $\zeta: \mathbb{R}^{d-1} \rightarrow[0,1]$ such that

$$
\begin{aligned}
& -\zeta(v)=1 \text { if } v \in\left[-\theta_{0}^{2}, \theta_{0}^{2}\right]^{d-1} \text { and } \\
& -\zeta(v)=0 \text { if } v \in \mathbb{R}^{d-1} \backslash\left[-\theta_{0}, \theta_{0}\right]^{d-1} ;
\end{aligned}
$$

- a smooth function $\xi:[-1,1] \rightarrow \mathbb{R}$ such that:

$$
\begin{aligned}
& -\xi(z)=z \text { for } z \in\left[-\theta_{0}^{2}, \theta_{0}^{2}\right], \\
& -\xi(z)=0 \text { for } z \text { in a neighborhood of }[-1,1] \backslash\left[-\theta_{0}, \theta_{0}\right], \text { and } \\
& -\xi(-z)=-\xi(z) .
\end{aligned}
$$

We denote by $X$ the vector field on $[-1,1]$ defined by $X(z)=\xi(z) \frac{\partial}{\partial z}$ and by $\left(X_{s}\right)_{s \in \mathbb{R}}$ the induced flow on $[-1,1]$. For every non-trivial closed interval $I \subset \mathbb{R}$ and any $s$, we denote by $X_{s}^{I}$ the diffeomorphism of $I$ obtained from $X_{s}$ by considering a parametrization of $I$ by $[-1,1]$ with constant derivative.

Remark 7.1. The distance $d_{C^{1}}\left(X_{s}^{I}, \mathrm{id}_{I}\right)$ has two parts. The $C^{0}$ part is proportional to the length of $I$. The part that measures the distance between the derivatives does not depend on $I$ :

$$
\max \left\{\left|D\left(X_{s}^{I}\right)-1\right|,\left|D\left(X_{-s}^{I}\right)-1\right|\right\}=\max \left\{\left|D\left(X_{s}\right)-1\right|,\left|D\left(X_{-s}\right)-1\right|\right\} .
$$

We fix constants $C, \varepsilon>0$ and a real number $s_{0}>0$ satisfying $\left.\max \left\{\mid D\left(X_{s_{0}}\right)-1\right)|| D,\left(X_{-s_{0}}\right)-1 \mid\right\}<\frac{\varepsilon}{C}$. We then define the diffeomorphisms $h_{s}=X_{s . s_{0}}$ and $h_{s}^{I}=X_{s . s_{0}}^{I}$.

Fix $K>0$. Note that the derivative of $h_{-1}$ on $\left[-\theta_{0}^{2}, \theta_{0}^{2}\right]$ is a constant: it is equal to $e^{-s_{\varepsilon}}<1$ for some constant $s_{\varepsilon}$. We fix $n_{2} \geq 1$ such that

$$
n_{2} s_{\varepsilon}>K
$$

\subsection{Construction of a perturbation}

Consider now a sequence of matrices $\left(A_{i}\right)_{i \in \mathbb{Z}}$ in $G L(d, \mathbb{R})$ such that $\|A\|,\left\|A_{i}^{-1}\right\|<C$, and denote by $f$ the associated linear cocycle. Up to a change of coordinates by isometries on each $\{i\} \times \mathbb{R}$, we may assume that the $A_{i}$ leave invariant the hyperplane $\mathbb{R}^{d-1} \times\{0\}$ and take the form

$$
A_{i}=\left(\begin{array}{llll} 
& & & \alpha_{1}^{i} \\
& B_{i} & & \vdots \\
& & & \alpha_{d-1}^{i} \\
0 & \ldots & 0 & b_{i}
\end{array}\right) .
$$

For $i \in\left\{0, \ldots, 2^{d+1} n_{2}\right\}$ and $\mu>0$ we set

$$
I_{i}=\left[-\mu \prod_{k=0}^{i-1} b_{k}, \mu \prod_{k=0}^{i-1} b_{k}\right] .
$$


We next fix $j \in\left\{0, \ldots, 2^{d}-1\right\}$. For each $i \in \mathbb{Z}$, we denote by $H_{i}$ the diffeomorphism of $\{i\} \times \mathbb{R}^{d}$ defined as follows:

- $H_{i}=$ id if $i \notin\left\{2 j n_{2}, \ldots, 2(j+1) n_{2}-1\right\}$.

- For $i \in\left\{2 j n_{2}, \ldots, 2(j+1) n_{2}-1\right\}$, the map $H_{i}$ coincides with id outside $\{i\} \times \mathbb{R}^{d-1} \times I_{i}$.

- For $i \in\left\{2 j n_{2}, \ldots, 2(j+1) n_{2}-1\right\}$ and $(v, z) \in \mathbb{R}^{d-1} \times I_{i}$, we set

$$
s(v)=\zeta\left(\left(B_{i-1} \ldots B_{0}\right)^{-1}(v)\right)
$$

and define

$$
\begin{aligned}
& H_{i}(v, z)=\left(v, h_{-s(v)}^{I_{i}}(z)\right) \text { if } i<(2 j+1) n_{2}, \\
& H_{i}(v, z)=\left(v, h_{+s(v)}^{I_{i}}(z) \text { if } i \geq(2 j+1) n_{2} .\right.
\end{aligned}
$$

Note that the diffeomorphisms $H_{i}$ depend on the choice of $\mu,\left(A_{i}\right)$ and $j$.

We define by $g$ the cocycle that coincides with $f \circ H_{i}$ on $\{i\} \times \mathbb{R}^{d}$. It will satisfy the conclusions of Proposition 6.6. provided $\mu$ has been chosen smaller than some constant $\mu_{0}=\min \left\{\mu_{1}, \mu_{2}, \mu_{3}, \mu_{4}\right\}$ which will be defined in the following subsections.

\subsection{Support of the perturbation: first choice of $\mu$}

Let $\mathcal{C}_{i}=P_{i} \times I_{i}$ where $P_{i}=B_{i-1} \ldots B_{0}\left([-1,1]^{d-1}\right)$. Let $Q_{i}=A_{i-1} \ldots A_{0}\left(Q_{\mu}\right)$. The two parallelepipeds $\mathcal{C}_{i}$ and $Q_{i}$ have the same base $P_{i} \times\{0\}$ in $\mathbb{R}^{d-1} \times\{0\}$ and the same height along the coordinate $z$, but $\mathcal{C}_{i}$ has been rectified.

In these notations $g$ is a perturbation of $f$ with support contained in $\bigcup_{i=0}^{2 n_{2}-1}\{i+$ $\left.2 j n_{2}\right\} \times \theta_{0} \mathcal{C}_{i+2 j n_{2}}$.

Lemma 7.2. Given $d, C, K, \varepsilon>0$ and $\theta \in(0,1)$, there exists $\mu_{1}=\mu_{1}(d, C, K, \varepsilon, \theta)$ such that, for every $\mu \in\left(0, \mu_{1}\right)$, for every sequence $\left(A_{i}\right)$ in $G L(d, \mathbb{R})$ with $\left\|A_{i}\right\|,\left\|A_{i}^{-1}\right\|<C$ and for every $i \in\left\{0, \ldots, 2^{d+1} n_{2}\right\}$, we have:

$$
\theta_{0} \mathcal{C}_{i} \subset Q_{i}
$$

In particular the perturbation $g$ defined in Section 7.2 is supported in

$$
\bigcup_{i=2 j n_{2}}^{2(j+1) n_{2}-1} f^{i}\left(\{0\} \times Q_{\mu}\right)=\bigcup_{i=2 j n_{2}}^{2(j+1) n_{2}-1} Q_{i} .
$$

Proof. Choose

$$
\mu_{1}<\left(2^{d+1} n_{2} C^{2^{d+2} n_{2}}\right)^{-1}\left(\theta_{0}^{-1}-1\right) .
$$

Consider a point $\left(v_{i}, z_{i}\right)$ in $\mathcal{C}_{i}$, and for $0 \leq k<i$, its image $\left(v_{k}, z_{k}\right)$ under $\left(A_{i-1} \ldots A_{k}\right)^{-1}$. We need to show that $\left(v_{0}, z_{0}\right)$ belongs to $\theta_{0}^{-1} Q_{\mu}$.

For the second coordinate, we have $z_{0}=z_{i} \prod_{k=0}^{i-1} b_{k}^{-1}$, and hence $z_{0}$ belongs to $I_{0} \subset \theta_{0}^{-1} I_{0}$. It remains to control the first coordinate: for each $k$, it decomposes as 
$v_{k}=B_{k}^{-1} v_{k+1}+w_{k}$ where $w_{k}$ is the projection on the first coordinate of $A_{k}^{-1}\left(0, z_{k+1}\right)$.

Note that

$$
\left\|w_{k}\right\| \leq C\left|I_{k+1}\right| \leq \mu C^{k+2} \leq \mu C^{i+1} .
$$

Decomposes $v_{k}$ as a sum $\tilde{v}_{k}+r_{k}$ where $\tilde{v}_{k}=\left(A_{i-1} \ldots A_{k}\right)^{-1}\left(v_{i}\right)$ belongs to $P_{k}$ and

$$
r_{k}=\sum_{j=k}^{i-1}\left(A_{j-1} \ldots A_{k}\right)^{-1} w_{j} .
$$

We thus have

$$
\left\|r_{k}\right\| \leq(i-k) C^{i-k-1} \max \left\{w_{j}\right\} \leq i C^{2 i} \mu_{1} .
$$

Since $v_{0}$ belongs to $P_{0}=[-1,1]^{d-1} \times\{0\}$, it is now enough to show that $\left\|r_{0}\right\|$ is smaller than the distance between the complement of $\theta_{0}^{-1} P_{0}$ and $P_{0}$. This distance is bounded from below by $\theta_{0}^{-1}-1$. Our choice of $\mu_{1}$ now implies that $v_{0}$ belongs to $\theta_{0}^{-1} P_{0}$, as required.

\subsection{Size of the perturbation: second choice of $\mu$}

We check here that the perturbation $g$ is $C^{1}$-close to $f$.

Lemma 7.3. Given $d, C, K, \varepsilon, \theta>0$ there exists $\mu_{2}=\mu_{2}(d, C, K, \varepsilon, \theta)$ such that for any $\mu \in\left(0, \mu_{2}\right)$, for any sequence $\left(A_{i}\right)$ in $G L(d, \mathbb{R})$ with $\left\|A_{i}\right\|,\left\|A_{i}^{-1}\right\|<C$ and for any $j \in\left\{0, \ldots, 2^{d}-1\right\}$, the diffeomorphisms $f$ and $g$ defined at Section 7.2 satisfy

$$
d_{C^{1}}(g, f)<\varepsilon \text {. }
$$

Proof. We choose

$$
\mu_{2}<\frac{\varepsilon}{C^{2^{d+2} n_{2}} s_{0}\|\xi\|\|D \zeta\|} .
$$

For all $i \in\left\{0, \ldots, 2^{d+1} n_{2}-1\right\}$, the quantity $\left\|D H_{i}\left(\frac{\partial}{\partial z}\right)-\frac{\partial}{\partial z}\right\|$ is bounded by the maximum of $\left|D h_{1}-1\right|$ and $\left|D h_{-1}-1\right|$ and thus is bounded by $\varepsilon / C$, by our choice of $s_{0}$. For any unit vector $u$ of $\mathbb{R}^{d-1} \times\{0\}$ we have

$$
\begin{aligned}
\left\|D H_{i}\left(\frac{\partial}{\partial u}\right)-\frac{\partial}{\partial u}\right\| & \leq\|\xi\|\|D \zeta\|\left\|\left(B_{i-1} \ldots, B_{0}\right)^{-1}\right\| s_{0} \mu \prod_{j=0}^{i-1} b_{i} \\
& \leq C^{2\left(2^{d+1} n_{2}-1\right)} s_{0} \mu\|\xi\|\|D \zeta\| .
\end{aligned}
$$

It follows that for $\mu<\mu_{2}$, we have $\| D H_{i}-$ id $\|<\frac{\varepsilon}{C}$, and consequently we obtain $\|D g-D f\|<\varepsilon$. 


\subsection{Perturbation of the jacobian: third choice of $\mu$}

We define the effective support of the perturbation as the set where

$$
|\log \operatorname{det} D f-\log \operatorname{det} D g|=|\log \operatorname{det} D H|=s_{\varepsilon} .
$$

By construction, this effective support contains $\bigcup_{i=0}^{n_{2}-1}\left\{i+2 j n_{2}\right\} \times \theta_{0}^{2} \mathcal{C}_{i+2 j n_{2}}$. We now prove that for small $\mu$ the orbits of the points in $\{0\} \times \theta Q_{\mu}$ under $g$ meet the effective support; this implies that the perturbation has the expected effect on the jacobian along these orbits.

Lemma 7.4. Given $d, C, K, \varepsilon, \theta>0$ there exists $\mu_{3}=\mu_{3}(d, C, K, \varepsilon, \theta)$ such that for any $\mu \in\left(0, \mu_{2}\right)$, for any sequence $\left(A_{i}\right)$ in $G L(d, \mathbb{R})$ with $\left\|A_{i}\right\|,\left\|A_{i}^{-1}\right\|<C$ and for any $j \in\left\{0, \ldots, 2^{d}-1\right\}$, the perturbation $g$ defined at Section 7.2 satisfies for every $i \in\left\{0, \ldots n_{2}-1\right\}$ :

$$
g^{i}\left(\left\{2 j n_{2}\right\} \times \theta Q_{2 j n_{2}}\right) \subset\left\{i+2 j n_{2}\right\} \times \theta_{0}^{2} \mathcal{C}_{i+2 j n_{2}} .
$$

In particular, for every $x \in\{0\} \times \theta Q_{\mu}$ one has

$$
\left|\log \operatorname{det} D f^{n_{2}}\left(f^{2 j n_{2}}(x)\right)-\log \operatorname{det} D g^{n_{2}}\left(f^{2 j n_{2}}(x)\right)\right|=n_{2} s_{\varepsilon}>K .
$$

Proof. The proof is quite similar to the proof of Lemma 7.2. We choose

$$
\mu_{3}<\left(2^{d+1} n_{2} C^{2^{d+2} n_{2}}\right)^{-1}\left(\theta_{0}^{-1}-1\right) .
$$

Let $\left(0, v_{0}, z_{0}\right)$ be a point in $\{0\} \times \theta Q_{\mu}$, and let $\left(i, v_{i}, z_{i}\right)$ be its image under $g^{i}$. We will show that $\left(v_{i}, z_{i}\right)$ belongs to $\theta_{0}^{2} \mathcal{C}_{i}$, for every $i \in\left\{0, \ldots,(2 j+1) n_{2}-1\right\}$.

For the second coordinate, we have $z_{i} \leq z_{0} \prod_{k=0}^{i-1} b_{k}$, since $H_{i}$ is the identity, for $i<2 j n_{2}$, and shrinks the second coordinate, for $i \in\left\{2 j n_{2}, \ldots,(2 j+1) n_{2}-1\right\}$. Hence $z_{i}$ belongs to $\theta I_{i} \subset \theta_{0}^{2} I_{i}$. It remains to control the first coordinate: $H_{i}$ has the form $(v, z) \mapsto\left(v, h_{i}(v, z)\right)$ and $v_{i}$ decomposes as $B_{i-1} v_{i-1}+w_{i}$ where $w_{i}$ is the projection on the first coordinate of $A_{i-1}\left(0, h_{i-1}\left(v_{i-1}, z_{i-1}\right)\right)$. Note that

$$
\left\|w_{i}\right\| \leq C\left\|h_{i-1}\left(v_{i-1}, z_{i-1}\right)\right\| \leq C\left|z_{i-1}\right| \leq C^{i} \mu_{3} \theta
$$

In particular, $v_{i}$ decomposes as a sum $\tilde{v}_{i}+r_{i}$, where $\tilde{v}_{i}=B_{i-1} \ldots B_{0}\left(v_{0}\right)$ belongs to $\theta P_{i}$ and

$$
r_{i}=\sum_{k=1}^{i} A_{i-1} \ldots A_{k} w_{k} .
$$

It follows that $\left\|r_{i}\right\| \leq i C^{i} \mu_{3} \theta$.

Since $\bar{v}_{i}$ belongs to $\theta P_{i}$, it is now enough to show that $\left\|r_{i}\right\|$ is smaller than the distance between the complement of $\theta_{0}^{2} P_{i}$ and $\theta P_{i}=\theta_{0}^{3} P_{i}$. This distance is bounded from below by $C^{-i} \theta\left(\theta_{0}^{-1}-1\right)$. Our choice of $\mu_{1}$ now implies that $v_{i}$ belongs to $\theta_{0}^{2} P_{i}$, as required. 


\subsection{Almost tidy perturbation: fourth choice of $\mu$}

To finish the proof of Proposition 6.6, we are left to show that, after a rescaling by the linear map $E_{\mu}$, the action of the perturbation $g$ on the orbits of $f$ tends uniformly to the identity as $\mu \rightarrow 0$.

Lemma 7.5. Given $d, C, K, \varepsilon, \theta$ and $\eta>0$ there exists $\mu_{4}=\mu_{4}(d, C, K, \varepsilon, \theta, \eta)$ such that for any $\mu \in\left(0, \mu_{4}\right)$, for any sequence $\left(A_{i}\right)$ in $G L(d, \mathbb{R})$ with $\left\|A_{i}\right\|,\left\|A_{i}^{-1}\right\|<C$ and for any $j \in\left\{0, \ldots, 2^{d}-1\right\}$, the diffeomorphisms $f$ and $g$ defined in Section 7.2 satisfy on $\{0\} \times \mathbb{R}^{d}$,

$$
d_{\text {unif }}\left(\mathrm{id}, E_{\mu}^{-1} f^{-2(j+1) n_{2}} g^{2(j+1) n_{2}} E_{\mu_{\{0\} \times \mathbb{R}^{d}}}\right)<\eta \text {. }
$$

Proof. First of all, notice that:

- $H_{i}=$ id if $i<2 j n_{2}$ or $i \geq 2(j+1) n_{2}-1$,

- $H_{i}=\left(E_{\mu} \bar{A}_{i-1} \ldots \bar{A}_{0}\right) H^{-1}\left(E_{\mu} \bar{A}_{i-1} \ldots \bar{A}_{0}\right)^{-1}$ for $2 j n_{2} \leq i<(2 j+1) n_{2}$,

- $H_{i}=\left(E_{\mu} \bar{A}_{i-1} \ldots \bar{A}_{0}\right) H\left(E_{\mu} \bar{A}_{i-1} \ldots \bar{A}_{0}\right)^{-1}$ for $(2 j+1) n_{2} \leq i<2(j+1) n_{2}$,

where $H$ is a diffeomorphism of $\{0\} \times \mathbb{R}^{d}$, and the $\bar{A}_{k}$ are matrices, defined by

$$
\begin{gathered}
H(v, z)=\left(v, h_{\zeta(v)}(z)\right), \\
\bar{A}_{k}=\left(\begin{array}{cccc} 
& & & 0 \\
& & & \vdots \\
& B_{k} & & 0 \\
& & & 0 \\
0 & \ldots & & b_{k}
\end{array}\right) .
\end{gathered}
$$

In this notation, we can write

$$
\begin{array}{r}
E_{\mu}^{-1} f^{-2(j+1) n_{2}} g^{2(j+1) n_{2}} E_{\left.\mu\right|_{\{0\} \times \mathbb{R}^{d}}}=E_{\mu}^{-1}\left(\prod_{i=0}^{2(j+1) n_{2}-1} f^{-i} H_{i} f^{i}\right) E_{\mu} \\
=Q_{l}\left(P_{2 n_{2}-1} H \ldots P_{n_{2}} H\right)\left(P_{n_{2}-1} H^{-1} \ldots P_{0} H^{-1}\right) Q_{r}
\end{array}
$$

where

$$
\begin{gathered}
P_{i}=\left(\bar{A}_{2 j n_{2}+i-1} \ldots \bar{A}_{0}\right)^{-1}\left(\bar{A}_{2 j n_{2}+i}^{-1} E_{\mu}^{-1} A_{2 j n_{2}+i} E_{\mu}\right)\left(\bar{A}_{2 j n_{2}+i-1} \ldots \bar{A}_{0}\right), \\
Q_{r}=\left(\bar{A}_{2 j n_{2}-1} \ldots \bar{A}_{0}\right)^{-1} E_{\mu}^{-1}\left(A_{2 j n_{2}-1} \ldots A_{0}\right) E_{\mu}, \quad \text { and } \\
Q_{l}=E_{\mu}^{-1}\left(A_{2(j+1) n_{2}-1} \ldots A_{0}\right)^{-1} E_{\mu}\left(\bar{A}_{2(j+1) n_{2}-1} \ldots \bar{A}_{0}\right) .
\end{gathered}
$$

Note that

$$
E_{\mu}^{-1} A_{k} E_{\mu} \underset{\mu \rightarrow 0}{\longrightarrow} \bar{A}_{k}
$$

This implies that $P_{i}, Q_{r}$ and $Q_{l}$ tend to $I_{d}$ when $\mu$ goes to 0 . As a consequence $E_{\mu}^{-1} f^{-2(j+1) n_{2}} g^{2(j+1) n_{2}} E_{\mu}$ tends uniformly to the identity on $\{0\} \times \mathbb{R}^{d}$ as $\mu \rightarrow 0$. This implies the conclusion of the lemma. 


\section{Appendix: The (LD) property is not generic}

The proof of our main theorem would have been much easier if the large derivative property were a generic property; the aim of this section is to show that, indeed, it is not a generic property.

Consider the set $L D \subset \operatorname{Diff}^{1}(M)$ of diffeomorphisms having the (LD)-property.

Remark 7.6. If $f$ is Axiom A, then $f \in L D$. More generally, if the chain recurrent set $C R(f)$ is a finite union of invariant compact sets, each of them admiting a dominated splitting, then $f \in L D$ : the dominated splitting implies that the vectors in one bundle are exponentially more expanded than the vectors in the other bundle, implying that $\sup \left\{\left\|D f^{n}(x)\right\|,\left\|D f^{-n}(x)\right\|\right\}$ increases exponentially with $n$ for $x \in$ $C R(f)$.

Consider the open set $\mathcal{T}$ of tame diffeomorphisms: these are the diffeomorphisms such that all the diffeomorphisms in a $C^{1}$-neighborhood have the same finite number of chain recurrence classes. A consequence of [BDP] and [BC] is that any diffeomorphism in an open and dense subset $\mathcal{O} \subset \mathcal{T}$ admits a dominated splitting on each of its chain recurrence classes. This implies that $\mathcal{O}$ is contained in $L D$.

To find an open set in which $L D$ is not residual, we therefore must look among the the so-called wild diffeomorphisms, whose chain recurrent set has no dominated splitting.

Proposition 7.7. For any compact manifold $M$ with $\operatorname{dim}(M)>2$, there exists a non-empty open subset $U_{M} \subset \operatorname{Diff}^{1}(M)$ such that $L D \cap U_{M}$ is meager.

Let $V_{M}$ be the set of diffeomorphisms $f$ possessing a periodic point $x=x_{f}$ such that $D f^{\pi(x)}(x)=i d$, where $\pi(x)$ is the period of $x$. We denote by $U_{M}$ the interior of the closure of $V_{M}$. In [BD, it is shown that $U_{M}$ is nonempty, for every compact manifold $M$ of dimension at least 3 .

Remark 7.8. The least period of $x_{f}$ is not locally bounded in $U_{M}$. This is because, for every $n>0$, the set of diffeomorphisms whose periodic orbits of period less than $n$ are hyperbolic is open and dense.

To prove the proposition, it suffices to show that $L D \cap U_{M}$ is meager.

For every $K>1$ and $n \in \mathbb{N}$, we consider the set $W(K, n) \subset \operatorname{Diff}^{1}(M)$ of diffeomorphisms $f$ such that there exist $m>n$ and two compact balls $B_{0}, B_{1}$ with the following properties.

- $B_{1}$ is contained in the interior of $B_{0}$;

- $f^{m}\left(B_{0}\right)$ is contained in the interior of $B_{0}$, and $B_{1}$ is contained in the interior of $f^{m}\left(B_{1}\right)$;

- for every $x \in B_{0}$ :

$$
\sup \left\{\left\|D f^{m}\left(f^{j}(x)\right)\right\|,\left\|D f^{-m}\left(f^{j} x\right)\right\|, x \in \Lambda_{f} \text { and } j \geq 0\right\}<K .
$$

Note that $W(K, n)$ is an open set, for every $K$ and $n$. Furthermore, for a given $K>1$, the diffeomorphisms in $\mathcal{R}=\cap_{n \in \mathbb{N}} W(K, n)$ do not satisfy the (LD) property. 
To see this, let $f \in \mathcal{R}$, and fix an arbitrary integer $n>0$. Consider an integer $m>n$ and two balls $B_{0}, B_{1}$ given by the definition of $W(K, n)$. Then the points in the open set $\operatorname{Int}\left(B_{1}\right) \backslash f^{-m}\left(B_{1}\right)$ are not periodic, since their entire orbits lie in $B_{0}$, and along these orbits, the quantities $\left\|D f^{m}\right\|$ and $\left\|D f^{-m}\right\|$ are bounded by $K$. Hence $f$ does not satisfy the (LD) property.

Lemma 7.9. For every $K>1$ and every $n \in \mathbb{N}, W(K, n) \cap U_{M}$ is (open and) dense in $U_{M}$.

Proof. Consider $f_{0} \in U_{M}$, and let $\mathcal{U} \subset U_{M}$ be a neighborhood of $f_{0}$. There exists $f_{1} \in \mathcal{U}$ and $m>n$ such that $f_{1}$ has a periodic point $x$ of period $m$ with $D f^{m}(x)=I d$. Then there exist $f_{2} \in \mathcal{U}$, arbitrarily close to $f_{1}$, and a small ball $D$ contained in an arbitrarily small neighborhood of $x$ such that $f_{2}^{m}(D)=D$, the restriction of $f_{2}^{m}$ to $D$ is the identity map, and $f_{2}^{i}(D) \cap D=\emptyset$ for $i \in\{1, \ldots, m-1\}$. Let $\Delta=\bigcup_{0}^{m} f_{2}^{i}(D)$.

Observe that there is a neighborhood $\mathcal{U}_{1} \subset \mathcal{U}$ of $f_{2}$ such that $\left\|D f^{m}\right\|$ and $\left\|D f^{-m}\right\|$ are bounded by $\frac{1}{2} K$ on $\Delta$. We conclude the proof by noting that there exist $f_{3} \in \mathcal{U}_{1}$ and two compact balls $B_{1} \subset \operatorname{Int}\left(B_{0}\right) \subset B_{0} \subset \operatorname{Int} D$ with the desired properties.

B. Fayad has observed that, for the proof of the Main Theorem, it suffices to obtain the (LD)-property merely on a dense subset of $M$. This weaker (LD) condition is not residual either: the proof of the proposition above shows that for the diffeomorphisms in the residual subset $\mathcal{R}$, the (LD)-property is not satisfied on any dense subset of $M$.

We conclude this section by discussing another strange feature of the (LD) property: we have proved the density of the set of diffeomorphisms satisfying the large derivative property and whose periodic orbits are all hyperbolic. On the one hand, the large derivative property is a uniform property on the non-periodic orbits (in the definition, the integer $n(K)$ does not depend on the point $x$ ). On the other hand, for the hyperbolic periodic orbits, the norm of the derivative tends exponentially to infinity. So it is natural to ask if we could also include the periodic orbits in the definition of (LD)-property. Let us say that $f$ satisfies the strong (LD) property if, for every $K>1$ there exists $n_{K}$ such that for every $n \geq n_{K}$ and every $x \in M$ one has $\sup \left\{\left\|D f^{n}\left(f^{i}(x)\right)\right\|, \| D f^{-n}\left(f^{i}(x) \|, i \in \mathbb{Z}\right\}>K\right.$.

Question 6. Does the strong (LD) property hold on a dense subset of $\operatorname{Diff}^{1}(M)$ ?

\section{References}

[BC] Bonatti, Ch.; Crovisier, S., Récurrence et généricité. Invent. Math. 158 (2004), 33-104.

[BCW1] Bonatti, Ch.; Crovisier, S.; Wilkinson, A., $C^{1}$-generic conservative diffeomorphisms have trivial centralizer. J. Mod. Dyn. 2 (2008), 359-373. A previous version was: Centralizers of $C^{1}$-generic diffeomorphisms. Preprint (2006) arXiv:math/0610064.

[BCW2] Bonatti, Ch.; Crovisier, S.; Wilkinson, A., The centralizer of a $C^{1}$ generic diffeomorphism is trivial. Preprint arXiv:0705.0225. 
[BCVW] Bonatti, Ch.; Crovisier, S.; Vago, G; Wilkinson, A., Local density of diffeomorphisms with large centralizers. Preprint (2007) arXiv:0709.4319.

[BD] Bonatti, Ch.; Díaz, L., On maximal transitive sets of generic diffeomorphisms. Publ. Math. Inst. Hautes Études Sci. 96 (2002), 171-197.

[BDP] Bonatti, Ch.; Díaz, L.; Pujals, E., A $C^{1}$-generic dichotomy for diffeomorphisms: weak forms of hyperbolicity or infinitely many sinks or sources. Ann. of Math. 158 (2003), 355-418.

[Bu1] Burslem, L., Centralizers of partially hyperbolic diffeomorphisms. Ergod. Th. $\&$ Dynam. Sys. 24 (2004), 55-87.

[Bu2] Burslem, L., Centralizers of area preserving diffeomorphisms on $S^{2}$. Proc. Amer. Math. Soc. 133 (2005), 1101-1108.

[Fi] Fisher, T., Trivial centralizers for Axiom A diffeomorphisms. Preprint.

[FRW] Foreman, M.; Rudolph, D.; Weiss, L., On the conjugacy relation in ergodic theory. C. R. Math. Acad. Sci. Paris 343 (2006), 653-656.

[G] Ghys, É., Groups acting on the circle. L'Enseign. Math. 47 (2001), 329407.

[Ko] Kopell, N., Commuting diffeomorphisms. In Global Analysis, Proc. Sympos. Pure Math., Vol. XIV, AMS (1970), 165-184.

[N] Navas, A., Three remarks on one dimensional bi-Lipschitz conjugacies. Preprint 2007, arXiv:0705.0034.

[P] Palis, J., Vector fields generate few diffeomorphisms. Bull. Amer. Math. Soc. 80 (1974), 503-505.

[PY1] Palis, J.; Yoccoz, J.-C., Rigidity of centralizers of diffeomorphisms. Ann. Sci. École Norm. Sup. 22 (1989), 81-98.

[PY2] Palis, J.; Yoccoz, J.-C., Centralizers of Anosov diffeomorphisms on tori. Ann. Sci. École Norm. Sup. 22 (1989), 99-108.

[Pu] Pugh, C., The closing lemma. Amer. J. Math. 89 (1967), 956-1009.

[R] Rees, M., A minimal positive entropy homeomorphism of the 2-torus. $J$. London Math. Soc. 23 (1981), 537-550.

[Sm1] Smale, S., Dynamics retrospective: great problems, attempts that failed. Nonlinear science: the next decade (Los Alamos, NM, 1990). Phys. D 51 (1991), 267-273.

[Sm2] Smale, S., Mathematical problems for the next century. Math. Intelligencer 20 (1998), 7-15. 
[To1] Togawa, Y., Generic Morse-Smale diffeomorphisms have only trivial symmetries. Proc. Amer. Math. Soc. 65 (1977), 145-149.

[To2] Togawa, Y., Centralizers of $C^{1}$-diffeomorphisms. Proc. Amer. Math. Soc. 71 (1978), 289-293.

Christian Bonatti (bonatti@u-bourgogne.fr)

CNRS - Institut de Mathématiques de Bourgogne, UMR 5584

BP 47870

21078 Dijon Cedex, France

Sylvain Crovisier (crovisie@math.univ-paris13.fr)

CNRS - Laboratoire Analyse, Géométrie et Applications, UMR 7539,

Institut Galilée, Université Paris 13, Avenue J.-B. Clément,

93430 Villetaneuse, France

Amie Wilkinson (wilkinso@math.northwestern.edu)

Department of Mathematics, Northwestern University

2033 Sheridan Road

Evanston, IL 60208-2730, USA 